Aus dem Fachbereich Medizin

der Johann Wolfgang Goethe-Universität

Frankfurt am Main

betreut am

Zentrum der Neurologie und Neurochirurgie (ZNN)

Klinik für Neurologie

Direktor: Prof. Dr. Helmuth Steinmetz

\title{
Charakterisierung mikrostruktureller zerebraler Pathologie bei fokaler idiopathischer Dystonie mittels quantitativer Magnetresonanztomographie
}

\author{
Dissertation \\ zur Erlangung des Doktorgrades der Medizin \\ des Fachbereichs Medizin \\ der Johann Wolfgang Goethe-Universität \\ Frankfurt am Main
}

vorgelegt von

Franca Natalie Machel

aus Berlin

Frankfurt am Main, 2020 
Dekan:

Referent:

Korreferent/in:

Tag der mündlichen Prüfung:
Prof. Dr. Stefan Zeuzem

PD Dr. Simon Baudrexel

Prof. Dr. Jochen Roeper

10.06.2021 


\section{Inhaltsverzeichnis}

Abbildungsverzeichnis ...........................................................................

Tabellenverzeichnis ..............................................................................IV

Abkürzungsverzeichnis .............................................................................. V

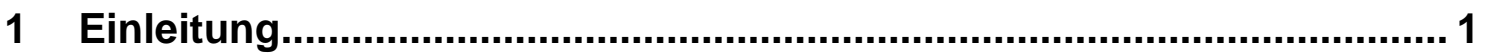

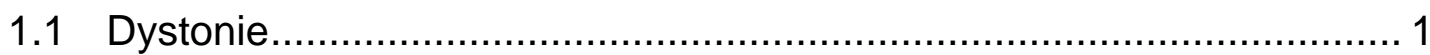

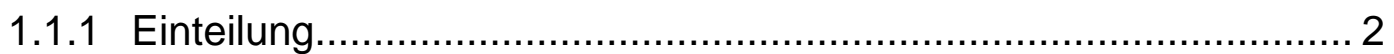

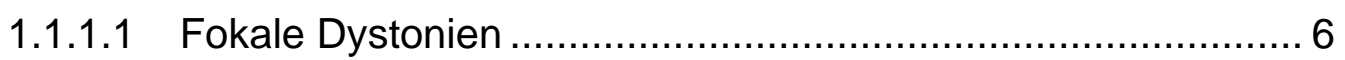

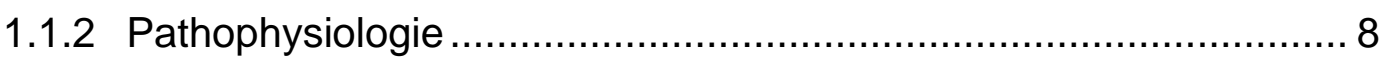

1.1.3 Diagnostik und Therapie ................................................ 11

1.2 Quantitative Magnetresonanztomographie ................................... 11

1.2.1 Zusammenhang zwischen qMRT-Parametern und Eigenschaften von Hirngewebe ............................................................. 12

1.2.2 Volumenabweichungen bei idiopathischen Dystonien................. 14

1.2.3 qMRT-Veränderungen bei idiopathischen Dystonien .................. 15

1.3 Fragestellung ....................................................................... 16

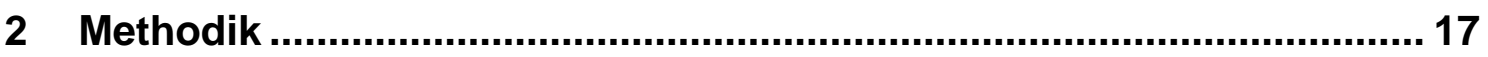

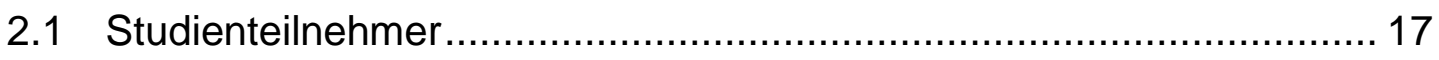

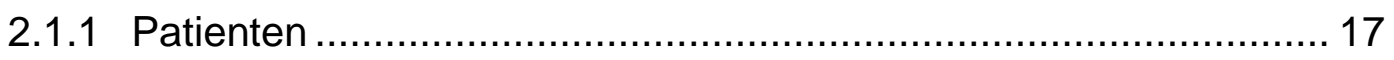

2.1.2 Kontrollprobanden ...................................................... 20

2.2 Überblick Datenerhebung und -auswertung ................................. 21

2.3 Magnetresonanztomographie ................................................. 22

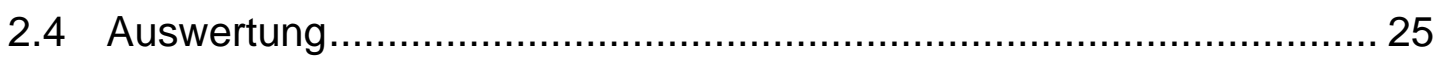

2.5 Dokumentation und Statistik ................................................ 28

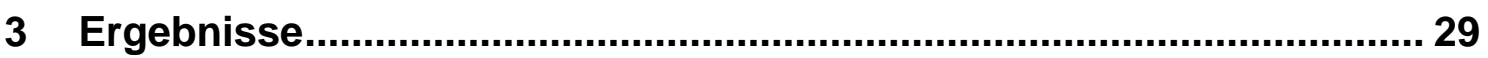

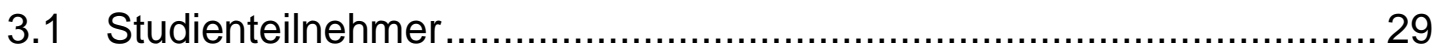




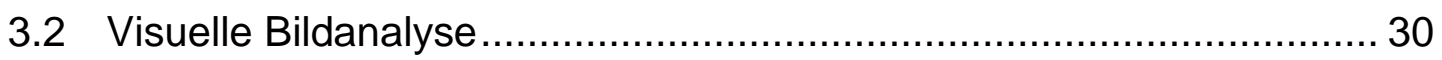

3.3 Resultate der Gewebesegmentierung …………........................... 30

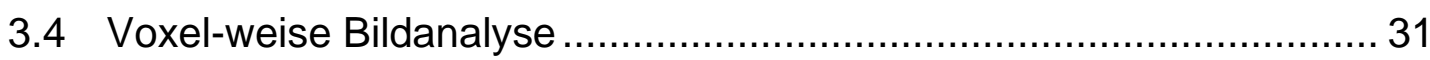

3.5 Region of Interest basierte Analyse ............................................... 33

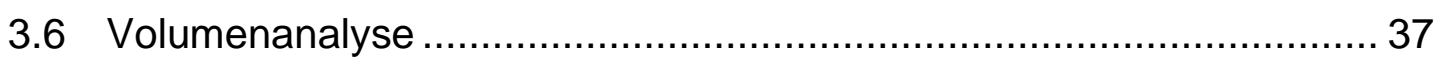

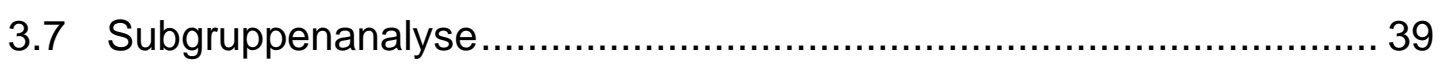

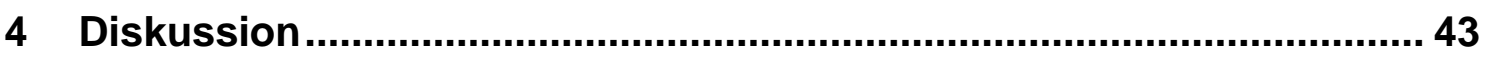

4.1 Quantitative Gewebeparameter .................................................... 43

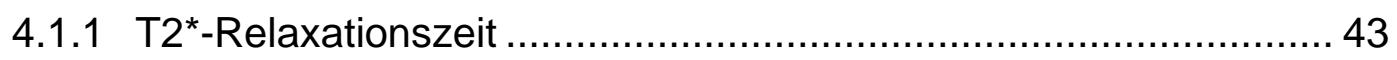

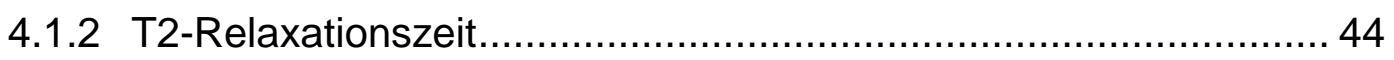

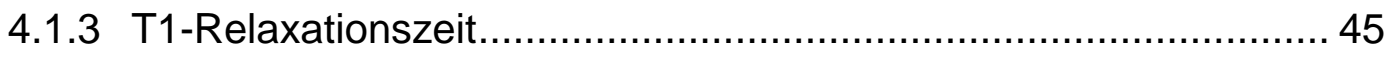

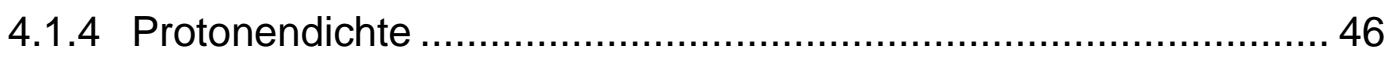

4.2 Verknüpfung bildgebender Studien mit der Histopathologie ................ 47

4.3 Volumenveränderungen bei idiopathischen Dystonien ........................ 49

4.4 Korrelation des klinischen Schweregrades mit bildgebenden Parametern ..................................................................... 50

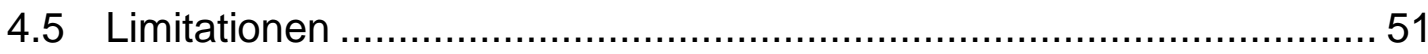

4.6 Idiopathische Dystonien als neurofunktionelle Störung …………....... 52

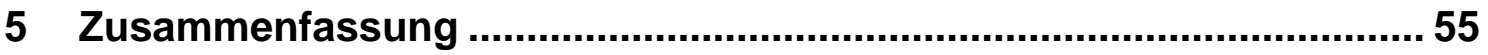

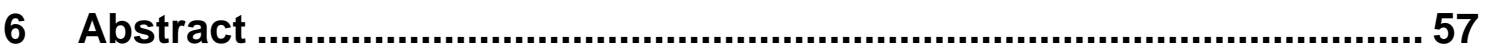

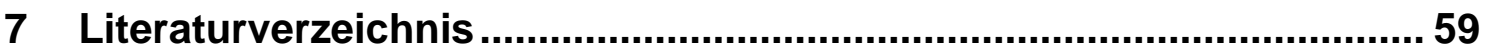

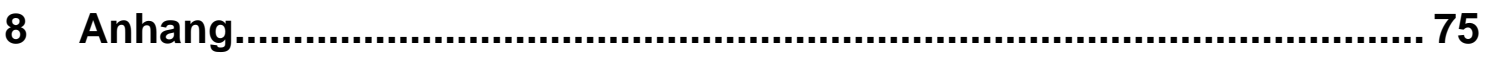

A.1 Toronto Western Spasmodic Torticollis Rating Scale (TWSTRS) ${ }^{95} \ldots .75$

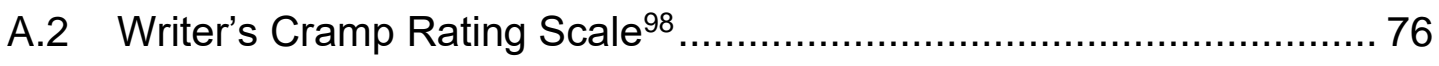

9 Eigene Veröffentlichungen.................................................................... 78

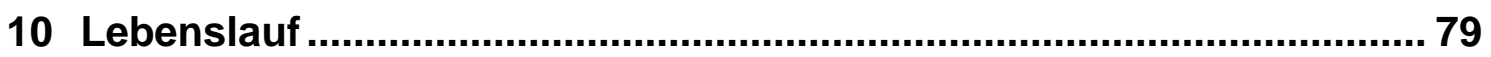

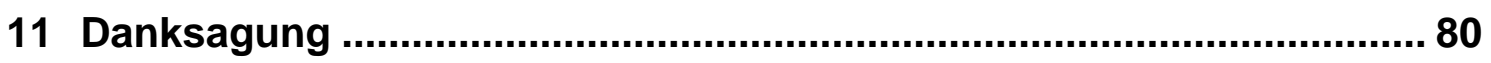

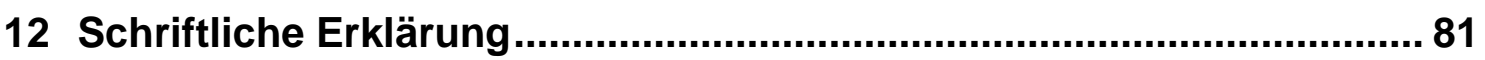




\section{Abbildungsverzeichnis}

Abbildung 1: Klinische Präsentation von Patienten mit fokaler Dystonie ............ 1

Abbildung 2: Klassisches Modell der motorischen Basalganglienfunktion ....... 10

Abbildung 3: Gewebesegmentierung der tiefen Grauen und Weißen Substanz

Abbildung 4: Quantitative T1-, T2/T2*- und PD-Karten 32

Abbildung 5: Unkorrigierter voxelbasierter t-Test für den T1- und PDGruppenvergleich 33

Abbildung 6.1: Ergebnisse der Messungen der T1-Relaxationszeit 34

Abbildung 6.2: Ergebnisse der Messungen der T2-Relaxationszeit 35

Abbildung 6.3: Ergebnisse der Messungen der T2*-Relaxationszeit 36

Abbildung 6.4: Ergebnisse der Messungen der Protonendichte .37

Abbildung 7: Ergebnisse der Volumenanalyse für verschiedene Regions of Interest

Abbildung 8: Ergebnisse der Subgruppenanalyse für Patienten mit zervikaler Dystonie

Abbildung 9: Ergebnisse der Volumenanalyse für Patienten mit zervikaler Dystonie

Abbildung 10: Streudiagramm von T1-Relaxationszeiten versus Tsui-Scores . 42 


\section{Tabellenverzeichnis}

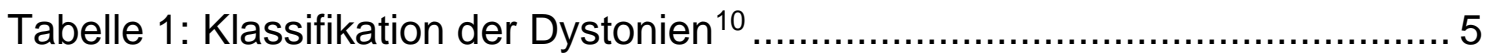

Tabelle 2: Patienten- und Probandencharakteristika im Vergleich ................... 17

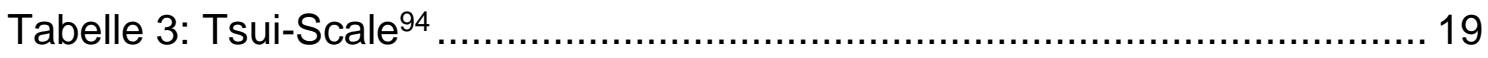

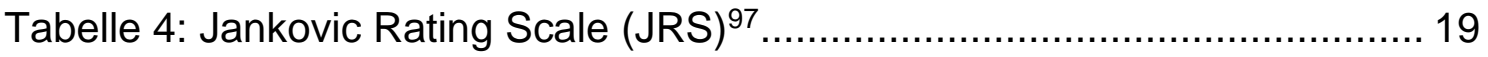

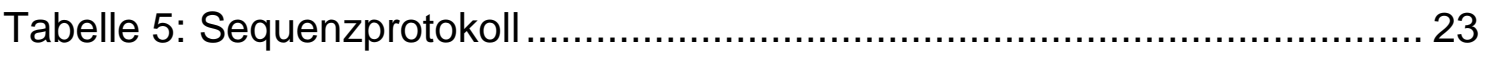

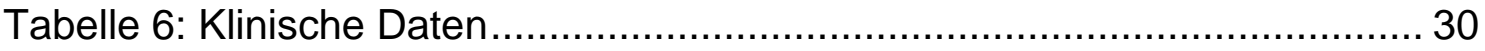




\section{Abkürzungsverzeichnis}

B0 statisches Magnetfeld des MRT-Scanners

B1 ausgesendetes Radiofrequenzfeld

DTI diffusion tensor imaging

FA fraktionelle Anisotropie

fMRT funktionelle Magnetresonanztomographie

FSL $\quad$ FMRIB Software Library

JRS Jankovic Rating Scale

MD mittlere Diffusivität

MP-RAGE magnetization-prepared rapid acquisition of gradient echoes

MRT Magnetresonanztomographie

Ncl. Nucleus

PD Protonendichte

qMRT quantitative Magnetresonanztomographie

ROI Region of Interest

TE Echozeit (engl. echo time)

TR Repetitionszeit (engl. repetition time)

TWSTRS Toronto Western Spasmodic Torticollis Rating Scale

VBM voxel-based morphometry (engl. für Voxel-basierte Morphometrie)

VFA variable flip angle 


\section{Einleitung}

\subsection{Dystonie}

Dystonien gehören zu den Bewegungsstörungen. Sie sind durch anhaltende oder intermittierende tonische Muskelkontraktionen gekennzeichnet, die zu abnormen, häufig repetitiven Körperhaltungen und/oder Bewegungen führen (siehe Abbildung 1) und regelmäßig mit Schmerzen verbunden sind ${ }^{1}$. Neben der Fehlfunktion in Bewegungs- und Haltungskontrolle in der betroffenen Region gehören auch Tremor und myoklonieähnliche Bewegungen zum Spektrum der dystonen Erkankungen ${ }^{2}$. Es zeigt sich zudem eine Assoziation zu psychiatrischen Erkrankungen wie beispielsweise Depressionen ${ }^{3}$ oder sozialer Phobien ${ }^{4}$. Die Prävalenz liegt bei 40/100.000 Einwohnern ${ }^{5}$. Damit sind sie die dritt häufigste Bewegungsstörung nach dem essentiellem Tremor und dem Morbus Parkinson ${ }^{6}$. Frauen sind signifikant häufiger betroffen als Männer ${ }^{7}$. Der Begriff der Dystonie geht ursprünglich auf Oppenheim zurück, der 1911 ein Syndrom als Dystonia musculorum deformans bezeichnete, welches er keiner anderen damals bekannten $\mathrm{Be}$ wegungsstörung zuordnen konnte ${ }^{8}$.
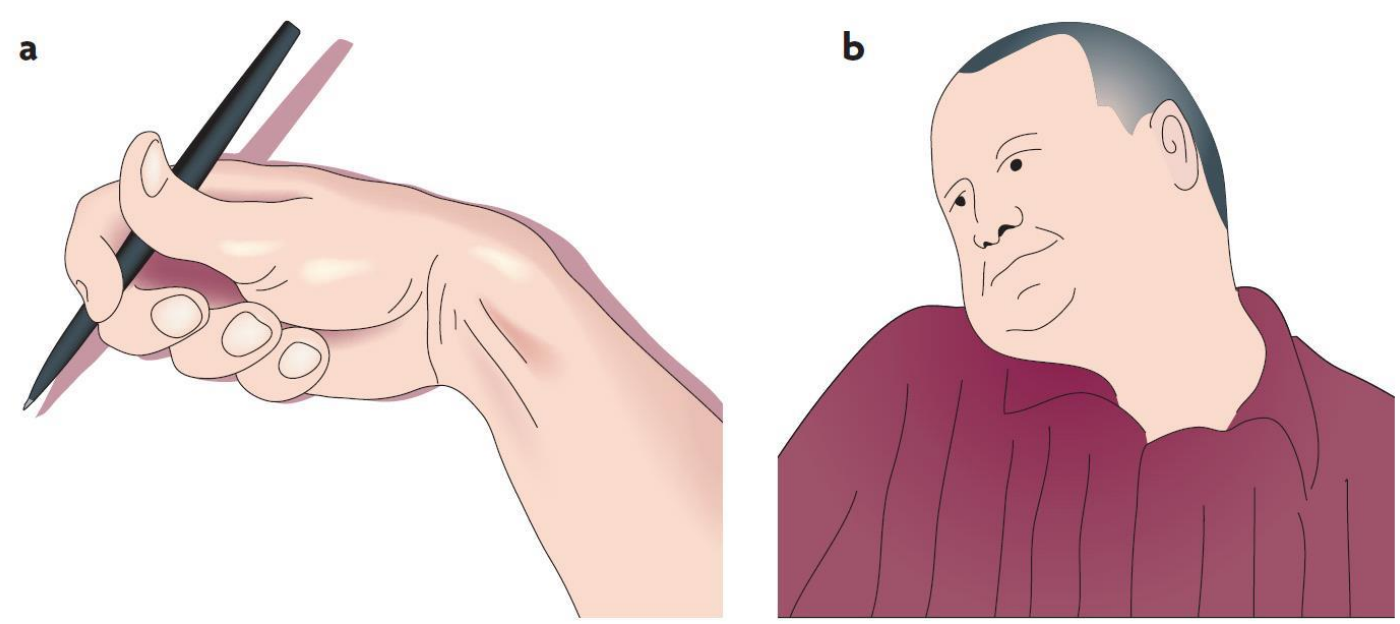

Abbildung 1: Klinische Präsentation von Patienten mit fokaler Dystonie

a) Patient mit Schreibkrampf; b) Patient mit zervikaler Dystonie; modifiziert nach Breakefield et al. ${ }^{9}$ 


\subsubsection{Einteilung}

Die Klassifikation der Dystonien findet entlang zweier Achsen statt ${ }^{10,11}$. Die erste Achse beschreibt die klinischen Charakteristika und den Phänotyp der Erkrankung. Es werden das Alter bei Erkrankungsbeginn, Symptomverteilung, zeitliches Muster und assoziierte Eigenschaften beurteilt (siehe Tabelle 1). Das Alter bei Erkrankungsbeginn kann auf die Ätiologie und somit auf die Prognose der Dystonie hinweisen. Beispielsweise kann eine Dystonie, die im Kleinkindesalter auftritt, mit einer Zerebralparese assoziiert sein, insbesondere wenn eine verzögerte motorische Entwicklung vorliegt ${ }^{10}$. Es werden fünf Alterskategorien angewandt: die Dystonie mit Beginn im Säuglingsalter (Geburt bis zwei Jahre), in der Kindheit (drei bis zwölf Jahre), im Jugendalter (13 bis 20 Jahre) sowie im frühen (21 bis 40 Jahre) und späten Erwachsenenalter (älter als 40 Jahre).

In Abhängigkeit von der klinischen Lokalisation der Symptome erfolgt die Einteilung in fokale, segmentale, multifokale, generalisierte und Hemidystonien ${ }^{10,}{ }^{12}$. Diese Unterteilung ist klinisch sehr bedeutend, da sie Einfluss auf die Therapie hat. Während bei fokalen Dystonien die lokale Injektion von Botulinum-Toxin (in der Regel Typ A) Therapie der ersten Wahl ist, werden generalisierte Dystonien mit Medikamenten (z.B. mit Anticholinergika) oder neurochirurgisch mittels Tiefer Hirnstimulation behandelt ${ }^{13}$. Prinzipiell kann jede Region des Bewegungsapparates, einschließlich des Pharynx und Larynx, von einer dystonen Bewegungsstörung betroffen sein. Fokal bedeutet, dass nur eine Körperregion beteiligt ist ${ }^{14}$. Zu den fokalen Dystonien zählen beispielsweise der Blepharospasmus, die oromandibuläre Dystonie und die zervikale Dystonie. Im Kapitel 1.1.1.1 Fokale Dystonien wird genauer auf die zugehörigen Krankheitsentitäten eingegangen. Eine segmentale Dystonie liegt vor, wenn zwei oder mehrere benachbarte Körperregionen Symptome zeigen ${ }^{10}$. Sind hingegen zwei oder mehrere nicht benachbarte Regionen betroffen, wird von einer multifokalen Dystonie geprochen². Bei den generalisierten Formen sind mindestens der Körperstamm und zwei weitere nicht benachbarte Körperregionen symptomatisch ${ }^{10}$. Die Hemidystonie ist darüber definiert, dass die Patienten dystone Symptome in mehrerer Körperregionen nur auf einer Körperseite zeigen ${ }^{12}$. 
Das zeitliche Muster beinhaltet den Krankheitsverlauf, welcher statisch als auch fortschreitend sein kann, und die Variabilität der Symptome, welche persistierend, aktionsspezifisch, täglich schwankend oder paroxysmal auftreten können ${ }^{15}$. Zudem findet eine Unterteilung in isolierte Dystonien, kombinierte Dystonien und Dystonien mit weiteren neurologischen oder systemischen Manifestationen statt ${ }^{10}$. Bei einer isolierten Dystonie tritt neben der dystonen Bewegungsstörung mit Ausnahme eines Tremors keine weitere Symptomatik auf. Ist die Dystonie mit einer weiteren Bewegungsstörung assoziiert, wie beispielsweise einer Myoklonie oder einem Parkinsonsyndrom, spricht man von einer kombinierten Dystonie. In die letzte Untergruppe werden Patienten mit zusätzlichen neurologischen oder systemischen Symptomen eingeteilt, die nicht den Bewegungsstörungen angehören. Ein Beispiel hierfür ist der Morbus Wilson, bei dem die dystonen Symptome typischerweise mit anderen neurologischen oder psychiatrischen Symptomen (z.B. Rigor oder Dysarthrie) sowie Lebererkrankungen kombiniert sind ${ }^{16}$.

Die zweite Achse dieser Klassifikation teilt nach der Ätiologie der Erkrankung ein. Bei vielen Formen der Dystonie ist die Ursache bisher nicht verstanden. Es flieBen zum einen identifizierbare anatomische Veränderungen (wie beispielsweise degenerative Veränderungen oder strukturelle Läsionen) und zum anderen das Vererbungsmuster in diese Achse ein (siehe Tabelle 1) ${ }^{11}$. Man unterscheidet zwischen angeborenen, erworbenen und idiopathischen Dystonien. Zu den angeborenen Dystonien zählen solche, bei denen eine genetische Mutation wie z.B. eine DYT1-Mutation nachgewiesen wird. Ursache einer erworbenen Dystonie können beispielsweise ein Zustand nach Schädelhirntrauma, Gefäßverletzungen, virale Infektionen oder Neoplasien sein. Weiterhin können die Einnahme verschiedener Medikamente (z.B. Antipsychotika, Calcium-Kanal-Blocker) oder Toxine (z.B. Kohlenstoffmonoxid) zu dystonen Symptomen führen ${ }^{9}$.

Die frühere ätiologische Klassifikation in primäre und sekundäre Dystonien ${ }^{17}$ wird somit verlassen. Die Diagnose einer primären Dystonieform erforderte per definitionem den bildgebenden Ausschluss einer erklärenden makroskopischen Pathologie ${ }^{18}$. Ebenfalls wurde unter "primär" das Fehlen von weiteren Symptomen mit Ausnahme eines Tremors verstanden ${ }^{19}$. Aufgrund dieser unklaren 
Terminologie, bei der es zu einer Vermischung von Ätiologie und Klinik kam, sind diese Begrifflichkeiten aufgegeben worden. Nach der aktuellen Klassifikation werden Patienten mit einer früher als primären Dystonie bezeichneten Unterform nun den isolierten idiopathischen Dystonien ohne nachweisbare strukturelle Veränderungen zugeordnet.

Im Folgenden wird sich die Arbeit auf fokale idiopathische Dystonien ohne nachweisbare strukturelle Veränderungen konzentrieren. 
Tabelle 1: Klassifikation der Dystonien ${ }^{10}$

\begin{tabular}{|c|c|c|}
\hline \multirow{2}{*}{\multicolumn{3}{|c|}{ Achse I - klinische Charakteristika }} \\
\hline \multirow[t]{2}{*}{ Alter bei Erkrankungsbeginn } & & \\
\hline & \multicolumn{2}{|c|}{$\begin{array}{l}\text { Säuglingsalter (Geburt bis zwei Jahre) } \\
\text { Kindheit (drei bis zwölf Jahre) } \\
\text { Jugendalter ( } 13 \text { - } 20 \text { Jahre) } \\
\text { frühes Erwachsenenalter ( } 21 \text { - } 40 \text { Jahre) } \\
\text { spätes Erwachsenenalter (> } 40 \text { Jahre) }\end{array}$} \\
\hline \multicolumn{3}{|l|}{ Symptomverteilung } \\
\hline & \multicolumn{2}{|l|}{$\begin{array}{l}\text { fokal } \\
\text { segmental } \\
\text { multifokal } \\
\text { generalisiert } \\
\text { Hemidystonien }\end{array}$} \\
\hline \multicolumn{3}{|l|}{ Zeitliches Muster } \\
\hline & Krankheitsverlauf & $\begin{array}{l}\text { statisch } \\
\text { fortschreitend }\end{array}$ \\
\hline & $\begin{array}{l}\text { Variabilität der } \\
\text { Symptome }\end{array}$ & $\begin{array}{l}\text { persistierend } \\
\text { aktionsspezifisch } \\
\text { täglich schwankend } \\
\text { paroxysmal }\end{array}$ \\
\hline \multicolumn{3}{|l|}{ Assoziierte Eigenschaften } \\
\hline & \multicolumn{2}{|c|}{$\begin{array}{l}\text { isolierte Dystonie } \\
\text { kombinierte Dystonie } \\
\text { Dystonie mit weiteren neurologischen oder } \\
\text { systemischen Manifestationen }\end{array}$} \\
\hline \multicolumn{3}{|l|}{ Achse II - Ätiologie } \\
\hline \multicolumn{3}{|l|}{ Anatomische Veränderungen } \\
\hline & \multicolumn{2}{|c|}{$\begin{array}{l}\text { Nachweis von Degeneration } \\
\text { Nachweis von strukturellen Läsionen } \\
\text { keine nachweisbaren Veränderungen }\end{array}$} \\
\hline \multicolumn{3}{|l|}{ Vererbungsmuster } \\
\hline & angeboren & $\begin{array}{l}\text { autosomal-dominant } \\
\text { autosomal-rezessiv } \\
\text { X-gekoppelt } \\
\text { mitochondrial }\end{array}$ \\
\hline & erworben & $\begin{array}{l}\text { perinatale Hirnschädigung } \\
\text { Infektion } \\
\text { Medikamente/Drogen } \\
\text { toxisch } \\
\text { vaskulär } \\
\text { neoplastisch } \\
\text { Hirnverletzung } \\
\text { psychogen }\end{array}$ \\
\hline & idiopathisch & $\begin{array}{l}\text { sporadisch } \\
\text { familiär }\end{array}$ \\
\hline
\end{tabular}




\subsubsection{Fokale Dystonien}

Die fokalen Dystonien stellen die häufigste Dystonieform im Erwachsenenalter dar ${ }^{20}$. Zu diesen zählen im Wesentlichen die zervikale Dystonie, der Blepharospasmus, das Meige-Syndrom und der Schreibkrampf. Seltener können auch pharyngeale oder laryngeale Dystonien auftreten. Die zervikale Dystonie, früher auch als Torticollis spasmodicus bezeichnet, ist die häufigste Form der fokalen Dystonien mit einer Prävalenz von 5,4 - 11,5/100.000 Einwohnern ${ }^{21,22}$. Die Erkrankung tritt gehäuft zwischen dem 30. und dem 50. Lebensjahr auf, wobei Frauen etwa doppelt so häufig betroffen sind wie Männer ${ }^{23}$. In der Regel ist keine Ursache feststellbar. Sie ist charakterisiert durch unwillkürliche Kontraktionen der Nacken-, Hals- und Schultermuskulatur. Dadurch kommt es zu unterschiedlichen Achsfehlstellungen des Kopfes. Beim Laterocollis wird der Kopf zur Seite geneigt, beim Anterocollis flektiert, beim Retrocollis extendiert und beim Torticollis gedreht. Im Allgemeinen entsteht eine kombinierte Kopffehlhaltung in den genannten Achsen ${ }^{24}$. Die zervikale Dystonie kann von einem Schulterhochstand, einem dystonen Kopftremor und Schmerzen begleitet sein ${ }^{25-27}$. Typischerweise kann die Symptomatik der Patienten durch einen sensorischen Trick, auch als geste antagonistique bezeichnet, gebessert werden (z.B. Anlegen eines Fingers an die Wange $)^{28}$. Emotionale Einflüsse wie beispielsweise Stress können die Symptomatik verstärken, während Schlaf und Entspannung die Symptome vermindern ${ }^{29}$. In der Regel ist die zervikale Dystonie eine chronische Erkrankung. Lediglich in 10 - 20\% der Fälle kann es zu einer Remission kommen, wobei nahezu alle Patienten innerhalb von fünf Jahren einen Rückfall erleiden ${ }^{30}$.

Der Blepharospasmus ist charakterisiert durch unwillkürliche Kontraktionen der periorbitalen, präseptalen und prätarsalen Muskulatur (Musculus orbicularis oculi $^{31}$. Es treten tonische, klonische oder tonisch-klonische bilaterale Kontraktionen auf, die zu wiederholtem und häufigem Zwinkern sowie zu anhaltendem Augenschließen führen ${ }^{32}$. In schweren Fällen kann es dadurch bis zu einer funktionellen Blindheit kommen. Die Symptomatik wird häufig durch helles Licht $^{33}$ oder starken Luftzug verstärkt, weshalb viele Patienten ständig eine Sonnenbrille tragen. Insbesondere zu Beginn der Erkrankung kann mittels bestimmter Tricks, 
wie beispielsweise Berühren der Augenbrauen, die Symptomatik abgemildert werden. Im Vergleich zu anderen Dystonieformen spielen diese aber eher eine untergeordnete Rolle. Der Blepharospasmus tritt gehäuft im mittleren bis höheren Lebensalter auf. Erkrankungsbeginn ist im Schnitt die fünfte bis siebte Lebensdekade $^{34}$. Er ist, wie auch die zervikale Dystonie, häufig idiopathisch ${ }^{35}$. Die Prävalenz liegt bei 1,6 - 13,3/100.000 Einwohnern ${ }^{36}$. Auch hier sind Frauen häufiger betroffen als Männer ${ }^{37}$. Über $60 \%$ der Patienten mit einem Blepharospasmus zeigen zusätzlich Symptome einer oromandibulären Dystonie ${ }^{38}$. Bei dieser kommt es zu unwillkürlichen Kontraktionen der Kiefer- und Mundmuskulatur. Häufig ist auch die mimische Muskulatur beteiligt. Symptome sind z.B. periorale Unruhe, Grimassieren sowie Schwierigkeiten bei der Nahrungsaufnahme. Tritt eine oromandibuläre Dystonie in Kombination mit einem Blepharospasmus auf, spricht man von einem Meige-Syndrom (Brueghel-Syndrom) ${ }^{39}$.

Der Schreibkrampf zählt zu der Gruppe der aktionsspezifischen fokalen isolierten Dystonien. Zu dieser gehören ebenfalls die Musiker- und Golferdystonie. Er ist durch unwillkürliche Kontraktionen und damit abnorme Haltung der Hand-, Finger- und/oder Armmuskeln während des Schreibens charakterisiert ${ }^{40}$. Dabei tritt der einfache Schreibkrampf ausschließlich beim Schreiben auf, während der dystone Schreibkrampf sich auch bei anderen Handtätigkeiten zeigt. Neben einer Prädisposition gehört auch das Wiederholen von stereotypen, feinmotorischen Bewegungen zu den Risikofaktoren ${ }^{41}$. Die Prävalenz liegt bei 1,4/100.000 Einwohnern ${ }^{42}$. Im Gegensatz zu anderen fokalen Dystonien sind mehr Männer als Frauen vom Schreibkrampf betroffen ${ }^{40}$. Der Erkrankungsbeginn liegt um das 40 . Lebensjahr ${ }^{40}$.

Eine weitere Form der fokalen isolierten Dystonie ist die spasmodische Dysphonie (larnygeale Dystonie), die sich durch eine aktionsinduzierte Kontraktion der Larynxmuskulatur bei Stimmbildung auszeichnet. Da keiner der in dieser Studie untersuchten Patienten diese Form der Dystonie zeigte, wird auf diese nicht weiter eingegangen. 


\subsubsection{Pathophysiologie}

Die Pathophysiologie fokaler idiopathischer Dystonien ist weitestgehend noch unverstanden und Gegenstand aktueller Forschung. Strukturen, die eine zentrale Rolle in der Genese spielen, sind die Basalganglien, die kortiko-striatothalamischen Regelkreise, der motorische Kortex sowie das Cerebellum und dessen Verknüpfungen zu den Basalganglien ${ }^{9}, 43,44$. Es kommt zu einer veränderten Kontrolle der Bewegungsplanung und -vorbereitung. Der Ort der Schädigung lässt sich auch aus den erworbenen Dystoniesyndromen ableiten. Ursachen dieser liegen häufig in den Basalganglien und zeigen eine Assoziation zu einzelnen oder kombinierten makroskopischen Läsionen des Putamen, Nucleus (Ncl.) caudatus, Globus pallidus oder posteroventraler Areale des Thalamus ${ }^{26,45,46}$. Es ist somit möglich, dass idiopathische Dystonien mit mikrostrukturellen Veränderungen assoziiert sind oder sogar in einem kausalen Zusammenhang stehen können.

Es liegen aktuell nur wenige Untersuchungen zu histopathologischen Veränderungen bei idiopathischen Dystonien vor. Die Ergebnisse sind heterogen und inkonsistent. Eine Fallserie zeigte z.B. Nervenzellverluste und das vermehrte Auftreten von neurofibrillary tangles, pathologischer intrazellulärer Proteinablagerungen sonst eigentlich dem Zytoskelett zugehöriger Filamente, in verschiedenen Kerngebieten des Hirnstammes ${ }^{47}$. In anderen Untersuchungen konnte eine reduzierte Zahl von pigmentierten Neuronen in der Substantia nigra ${ }^{6}$ oder ein Verlust von Purkinje-Zellen sowie Areale fokaler Gliose im Cerebellum ${ }^{48}$ nachgewiesen werden. In einer Studie von Gibb et al. hingegen fanden sich keine histopathologischen Veränderungen ${ }^{49}$. Insgesamt konnten bislang keine eindeutig übereinstimmenden mikrostrukturellen Veränderungen bestätigt werden.

Das funktionelle Modell der Basalganglien ${ }^{50,51}$ beschreibt die motorische Kontrolle von Bewegung. Die basalganglien-thalamokortikale Schleife reguliert neuronale Netzwerke des Motorkortex. Sie führt zu einer Aktivierung von Neuronen, die für eine Bewegung gebraucht werden, und gleichzeitig zu einer Suppression von Neuronen, die konkurrierende Bewegungen regulieren ${ }^{52}$. Es werden zwei Wege unterschieden: die direkte Schleife (Striatum - Globus pallidus internus - 
Thalamus) und die indirekte Schleife (Striatum - Globus pallidus externus - Ncl. subthalamicus - Globus pallidus internus - Thalamus, siehe Abbildung 2a). Das Striatum wird durch glutamaterge Afferenzen des Kortex erregt. Die direkte Schleife führt über inhibierende GABAerge Neurone des Striatums zu einer verminderten Aktivität des Globus pallidus internus. Dies führt zu einer Enthemmung der Thalamusneurone, die gewöhnlich einer partiellen konstitutiven Hemmung durch GABAerge Neurone des Globus pallidus internus unterliegen. Der Thalamus wiederum projiziert exzitatorisch in den Kortex. Die direkte Schleife fördert somit Bewegung ${ }^{53}$. Die indirekte Schleife hingegen unterdrückt Bewegung ${ }^{53}$. Das Striatum führt hier zu einer verstärkten Inhibition des Globus pallidus externus, wodurch die konstitutive Hemmung des Ncl. subthalamicus durch diesen vermindert wird. Dieser führt nun über exzitatorische Projektionsneurone zu einer erhöhten Aktivität des Globus pallidus internus. Dadurch wird die Hemmung des Thalamus verstärkt und Bewegung unterdrückt. Die Balance zwischen den beiden Schleifen wird über dopaminerge Neurone der Substantia nigra geregelt. Bei einem hyperkinetischen Syndrom, wie beispielsweise der Dystonie, scheint nun die direkte Schleife überaktiv zu sein ${ }^{51}$. Durch die erhöhte Aktivität des Striatums wird der Globus pallidus internus verstärkt inhibiert, was zu einer Enthemmung des Thalamus und nachfolgend des motorischen Kortex führt (Abbildung 2b). Es entsteht eine abnormale Kokontraktion von agonistischen und antagonistischen Muskelgruppen und somit dystone Symptome ${ }^{54}$. 


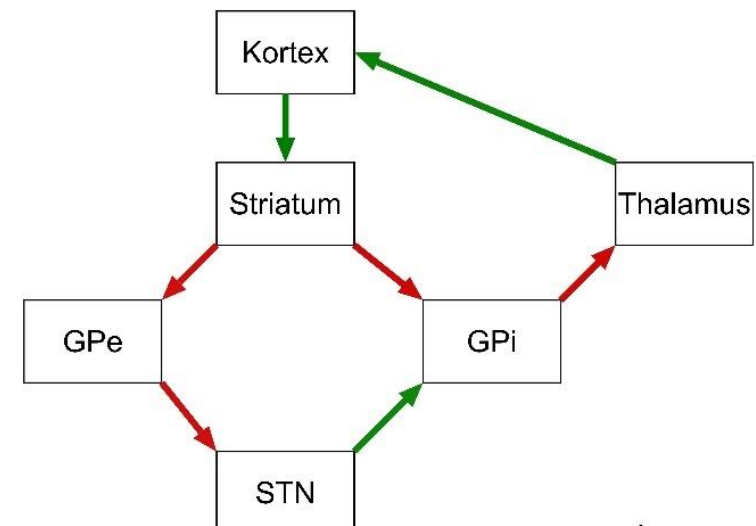

a)

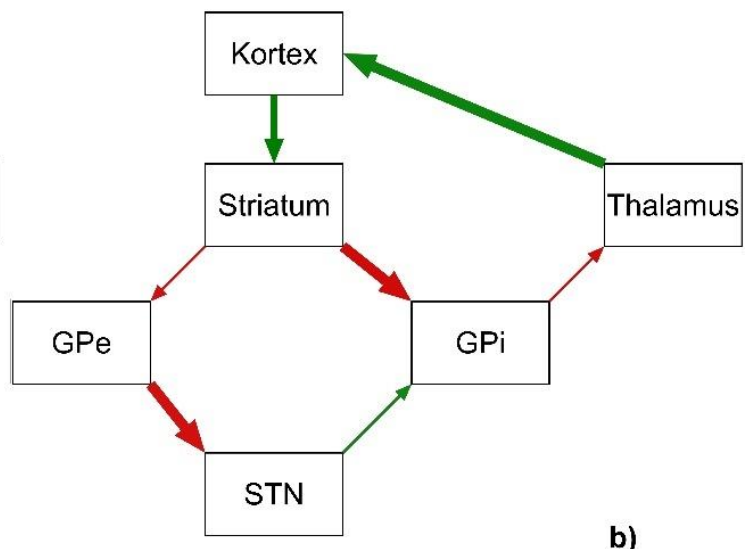

b)

$\underset{\text { Exzitatorisch }}{\longrightarrow} \underset{\text { Inhibitorisch }}{\longrightarrow}$

Abbildung 2: Klassisches Modell der motorischen Basalganglienfunktion

a) Verschaltung der Basalganglienkerne bei einem Gesunden; b) Verschaltung der Basalganglienkerne bei einer Hyperkinese. Die direkte Schleife ist verstärkt aktiv, die indirekte Schleife weniger aktiv. Dies führt zu einer verringerten Aktivität des GPi und verstärktem Output des Thalamus. GPi/GPe = Globus pallidus internus/externus, STN = Nucleus subthalamicus.

In einigen Studien konnten funktionelle Veränderungen v.a. im Bereich der Basalganglien nachgewiesen werden. Mittels funktioneller Magnetresonanztomographie (fMRT) können Veränderungen der Oxygenierung des Blutes dargestellt werden. Somit werden Durchblutungsänderungen von Hirnarealen und folglich deren Aktivität veranschaulicht ${ }^{43}$. Mittels dieser Methode ließ sich eine gesteigerte Aktivität im Bereich des Putamens und Pallidums bei Patienten mit Schreibkrampf nachweisen ${ }^{55}$. Neben der abnormalen Stoffwechselaktivität in den Basalganglien wurden untypische Aktivierungsmuster im sensomotorischen Kortex und im Cerebellum aufgezeigt ${ }^{56,57}$.

Zusammenfassend bleibt die Pathophysiologie idiopathischer Dystonien ungeklärt. Histopathologische Untersuchungen sowie das funktionelle Modell der Basalganglien unterstützen die Hypothese einer veränderten Mikrostruktur verschiedener in der motorischen Kontrolle involvierter Hirnareale. 


\subsubsection{Diagnostik und Therapie}

Im Mittelpunkt der Diagnostik von fokalen idiopathischen Dystonien stehen die ausführliche Anamnese und klinische Untersuchung. Zur Abklärung gehören auch eine kraniale Magnetresonanztomographie (MRT) und eine umfangreiche Labordiagnostik, um symptomatische Ursachen der Dystonie wie beispielsweise einen Morbus Wilson oder Neoplasien auszuschließen.

Aktuell stehen keine kurativen Therapiemöglichkeiten zur Verfügung. Ziel der Therapie ist folglich die Linderung der Symptomatik und Verbesserung der Lebensqualität. Therapie der Wahl bei fokalen Dystonien ist die lokale Injektion von Botulinumtoxin $A^{1}$, einem Neurotoxin, welches zu einer selektiven peripheren Denervierung der betroffenen Muskelgruppen führt. Die Behandlung kann durch die Gabe von Anticholinergika ergänzt werden ${ }^{13}$. Bei Therapierefraktion und gleichzeitig schwerer Symptomatik können neurochirurgische Verfahren, allen voran die Tiefe Hirnstimulation, oder eine medikamentöse Kombinationstherapie in Betracht gezogen werden ${ }^{13}$. Die Therapie wird in der Regel durch Physio- oder Ergotherapie ergänzt.

\subsection{Quantitative Magnetresonanztomographie}

Mit Methoden der sogenannten quantitativen Magnetresonanztomographie (qMRT) ist es möglich, am lebenden Probanden verschiedene physikalische Eigenschaften von Hirngewebe räumlich hochauflösend zu messen ${ }^{58}$. Zu den am häufigsten untersuchten Parametern gehören die Protonendichte (PD), die longitudinale Relaxationszeit $\mathrm{T} 1$ und die transversalen Relaxationszeiten T2/T2* (siehe Kapitel 1.2.1 Zusammenhang zwischen qMRT-Parametern und Eigenschaften von Hirngewebe). Sie alle hängen von der genauen mikrostrukturellen Zusammensetzung des Gewebes, wie beispielsweise dessen Wasser-, Myelinund Eisengehalt ab. Da es sich um wohldefinierte physikalische Größen handelt, können dreidimensionale Parameterkarten von verschiedenen Probanden mit statistischen Methoden analysiert werden, um so z.B. erkrankungsbedingte Unterschiede zwischen gesunden Personen und einem Patientenkollektiv in 
verschiedenen Hirnarealen zu identifizieren. Konventionelle T1-, T2/T2*- oder PD-gewichtete MRT-Bilder, wie sie üblicherweise auch in der klinischen Routine akquiriert werden, setzen sich aus einer Mischung der oben genannten Kontraste zusammen (Mischkontraste). Zudem werden die Intensitäten einzelner Bilddatenpunkte durch verschiedene externe Störgrößen, wie z.B. Inhomogenitäten des statischen Magnetfeldes (B0), der Intensitätsverteilung des während der Messung ausgesendeten Radiofrequenzfeldes (B1) oder der Sensitivität der Empfangsspule beeinflusst ${ }^{59,60}$. Daher eignen sich konventionelle MRT-Bilder nicht für statistische Gruppenvergleiche, insbesondere können subtile Veränderungen eines bestimmten Gewebeparameters nicht erfasst werden. Rückschlüsse auf zugrunde liegende mikrostrukturelle Pathologien sind daher nur eingeschränkt möglich. Dagegen liefert die qMRT quantitative Parametermaps, die jeweils ausschließlich Informationen über einen einzelnen Gewebeparameter anzeigen ${ }^{61}$, ohne einem Bias durch andere Gewebeparameter sowie der Beeinflussung durch Hardware-Parameter zu unterliegen ${ }^{62}$. Sie reagiert sensitiv auf Änderungen von Gewebeeigenschaften wie beispielsweise dem Gehalt an Myelin, Eisen sowie Wasser und eignet sich folglich zur Untersuchung krankheitsassoziierter Prozesse, die mit mikrostrukturellen Pathologien einhergehen ${ }^{63}$. Bereits mehrfach wurde diese Methode zur Detektion derartiger Veränderungen angewendet.

\subsubsection{Zusammenhang zwischen qMRT-Parametern und Eigenschaften von Hirngewebe}

Die Messung der T1- und T2/T2*-Relaxationszeiten sowie der PD erlaubt indirekte Rückschlüsse über mikrostrukturelle Gewebeeigenschaften. So korreliert die PD eng mit dem Wassergehalt von Gewebe ${ }^{64}$. Mittels der quantitativen Messung der PD konnte beispielsweise eine signifikante Erhöhung des globalen Wassergehaltes der Weißen Substanz bei Patienten mit hepatischer Enzephalopathie nachgewiesen werden ${ }^{65}$. Dies war der erste quantitative Nachweis eines leichtgradigen zerebralen Ödems bei dieser Erkrankung. 
Die longitudinale Relaxationszeit T1 wird hauptsächlich durch den Gehalt von Wasser sowie der Fett- und Proteinzusammensetzung des Gewebes beeinflusst. Änderungen von T1 können auf Prozesse, die mit Demyelinisierung, Gliose und axonalem Schaden zusammenhängen, hinweisen ${ }^{66}$. Ein erhöhter Wassergehalt führt dabei zu einer Verlängerung der T1-Relaxationszeit ${ }^{67}$, während ein erhöhter Myelingehalt diese verkürzt ${ }^{68}$. So konnten mittels Messung der T1-Relaxationszeit bei Patienten mit einem frühen Stadium einer Multiplen Sklerose bereits heterogene Veränderungen des gesamten Kortex auch außerhalb der leicht sichtbaren fokalen Läsionen der Weißen Substanz detektiert werden ${ }^{69}$. Die erhöhten T1-Werte wurden als Indikator eines diffusen inflammatorischen Prozesses und damit einhergehendem heterogenen kortikalen Schaden interpretiert.

Die transversale Relaxationszeit T2 lässt hauptsächlich Rückschlüsse über den Grad der Myelinisierung $\mathrm{zu}^{70}$. Sie wird allerdings auch durch den Eisen- und Wassergehalt eines Gewebes beeinflusst ${ }^{70}$. Auch hier führt ein erhöhter Wassergehalt zu einer verlängerten T2-Relaxationszeit ${ }^{71}$. Erhöhte T2-Werte treten z.B. im Rahmen eines Ödems, Demyelinisierung, axonalem Verlust oder Nekrose auf. So konnten beispielsweise in einer Untersuchung verkürzte T2-Zeiten im Tumorumgebenden Ödem von malignen Gliomen nachgewiesen werden ${ }^{72}$. Die Veränderungen könnten auf diffuse Tumorinfiltrate hinweisen. Diese detektierten Gewebeveränderungen waren im konventionellen MRT nicht sichtbar.

Die transversale Relaxationszeit T2* ist sensitiv gegenüber Inhomogenitäten des lokalen Magnetfeldes, welche durch paramagnetische Substanzen wie Eisen hervorgerufen werden ${ }^{63}$. Somit kann der Eisengehalt eines Gewebes abgeschätzt werden. Sie kombiniert dabei die transversale Relaxation mit der Relaxation, die durch Inhomogenitäten im Magnetfeld entsteht ${ }^{73}$. T2 sowie T2 ${ }^{*}$ zeigen eine inverse Korrelation zum Eisengehalt im Gewebe ${ }^{74,}$ 75. Die T2*-Zeit zeigt dementsprechend Veränderungen bei Erkrankungen, die mit einem modifizierten Eisengehalt einhergehen (siehe Kapitel 1.2.3 qMRT-Veränderungen bei idiopathischen Dystonien). 
Die kombinierte Messung dieser quantitativen Gewebeparameter bietet somit die Möglichkeit, verschiedene physikalische Aspekte des Gewebes zu beleuchten und auf Änderungen bestimmter Gewebeeigenschaften rückzuschließen.

\subsubsection{Volumenabweichungen bei idiopathischen Dystonien}

In der Regel zählen die fokalen Dystonien ätiologisch in die Gruppe der idiopathischen Dystonien ohne nachweisbare strukturelle Veränderungen. Dennoch konnten bereits in einigen Studien mittels konventioneller MRT und Voxel-basierter Morphometrie (engl. voxel-based morphometry, VBM ${ }^{76}$ anatomische Anomalien nachgewiesen werden. VBM ist eine Bildtechnik, die einen Vergleich von regionalen Gehirnvolumina erlaubt ${ }^{77}$. Normalerweise werden konventionelle T1-gewichtete Datensätze genutzt, um eine Gruppe von Patienten mit einer Kontrollgruppe zu vergleichen. Dadurch können quantitative Unterschiede im Volumen der Grauen und Weißen Substanz in spezifischen Arealen ermittelt werden, die mit bloßem Auge nicht erkennbar sind. Mithilfe von VBM konnte in einigen Studien ein abnormales Volumen in den Basalganglien, dem Thalamus, zerebralem Kortex und Cerebellum bei fokaler Dystonie gezeigt werden. So wiesen Patienten mit Blepharospasmus ${ }^{78}$ und Schreibkrampf ${ }^{79}$ vergrößerte Volumina des Putamens im Vergleich zu einer Kontrollkohorte auf. Andere Untersuchungen legten hingegen ein signifikant erniedrigtes putaminales Volumen bei Patienten mit zervikaler Dystonie und Blepharospasmus nahe ${ }^{80-82}$. Des Weiteren wurden Volumenänderungen in anderen Kerngebieten der Basalganglien ${ }^{83,84}$, insbesondere im Globus pallidus internus, im Cerebellum ${ }^{80,83}$ und im Thalamus ${ }^{85}$ beschrieben. Weiterhin konnte eine Erhöhung des Volumens der Grauen Substanz im primären sensomotorischen Kortex nachgewiesen werden ${ }^{83,86}$. Ein erhöhtes Volumen der Grauen Substanz wurde mit einer erhöhten Anzahl von Neuronen oder Synapsen ${ }^{78}$, als Resultat neuronaler Plastizität durch eine chronische Überaktivierung ${ }^{83}$ oder genetische und/oder epigenetische Faktoren, die die Patienten vulnerabler für Umweltfaktoren machen ${ }^{86}$, erklärt. Erniedrigte Volumina der Grauen Substanz ließen die Autoren auf eine Verringerung der neuronalen Dichte, Zellverlust und Atrophie der Zellen rückschließen ${ }^{81,82}$. Insgesamt sind die 
Ergebnisse der bisherigen Bildgebungsstudien zu volumetrischen Veränderungen bei fokalen Dystonien heterogen und teils widersprüchlich. Jedoch unterstützen sie zusammen mit den wenigen histopathologischen Studien die in dieser Arbeit verfolgte Hypothese, dass den idiopathischen fokalen Dystonien mikrostrukturelle Veränderungen zugrunde liegen könnten und zwar speziell in den oben genannten für die Bewegungskontrolle zuständigen Regionen (Basalganglien, Thalamus, Cerebellum, motorischer Kortex).

\subsection{3 qMRT-Veränderungen bei idiopathischen Dystonien}

Die überwiegende Mehrheit bisheriger kernspintomographischer Untersuchungen zur Gewebemikrostruktur bei Dystonien benutzten eine Methodik, die auf der Bestimmung der Diffusionseigenschaften von Wasser basiert, das sog. diffusion tensor imaging (DTI). Durch die Bestimmung des Diffusionsvermögen (mittlere Diffusivität, MD) ${ }^{87}$ und der Direktionalität der Diffusion (fraktionelle Anisotropie, $F A)^{88}$ von Wassermolekülen können Rückschlüsse über die Zelldichte, den Faserverlauf und den Ordnungsgrad der Gewebearchitektur getroffen werden ${ }^{43}$. Abweichungen der FA können durch multiple Faktoren wie z.B. Veränderung der Axondichte, der Membranintegrität der Axone, des Axondurchmessers, des Grades der Myelinisierung und der Kohärenz der Faserorientierung entstehen ${ }^{89}$. Mit dieser Methodik konnten Hinweise auf veränderte zerebrale Mikrostrukturen in den Basalganglien, dem Cerebellum, Motorkortex und der Weißen Substanz bei idiopathischen Dystonien gefunden werden. Carbon et al. wiesen u.a. Erniedrigungen der FA im Cerebellum und der Weißen Substanz sensomotorischer Regionen bei Patienten mit idiopathischer Torsionsdystonie nach ${ }^{88}$. Andere Arbeiten zeigten darüber hinaus Abweichungen der FA und MD im Putamen, Pallidum, Ncl. caudatus und einigen kortikalen Arealen bei Patienten mit zervikaler Dysto$n^{8} \mathrm{e}^{87}$, 90. Diese Veränderungen implizieren eine abnormale axonale Kohärenz bzw. Integrität und somit eine strukturelle Störung der normalen Architektur der Weißen Substanz. Zusammenfassend weisen die Daten auf mikrostrukturelle Abweichungen in verschiedenen Dystonie-relevanten Regionen hin, die derzeitige Studienlage ist allerdings heterogen mit teils inkonsistenten Resultaten. 
Dystonien wurden bislang nur in sehr wenigen Studien mit qMRT-Relaxometrie untersucht. In einer Pilotstudie mit 12 Patienten mit zervikaler Dystonie konnten mittels T2*-Mapping im Globus pallidus, nicht jedoch in anderen Basalganglienstrukturen, signifikant erniedrigte T2*-Werte im Vergleich zu einem gesunden Kontrollkollektiv nachgewiesen werden ${ }^{91}$. Dies impliziert erhöhte Eisenwerte im Globus pallidus. In einer anderen Untersuchung von 17 Patienten mit zervikaler Dystonie fanden sich hingegen verlängerte T2-Relaxationszeiten im Putamen und Pallidum ${ }^{92}$. Dies widerspricht der Annahme eines erhöhten Eisengehaltes im Gewebe und weist eher auf eine fokale Gliose und Zellverlust hin (siehe Kapitel 1.2.1 Zusammenhang zwischen qMRT-Parametern und Eigenschaften von Hirngewebe). Histologische Studien konnten bislang keine Veränderungen des Eisengehaltes nachweisen. Die widersprüchlichen Ergebnisse der qMRT-Studien lassen die Frage offen, ob der Eisengehalt in den Basalganglien tatsächlich erhöht ist und somit auch die Frage, ob Eisenablagerungen relevanter Bestandteil des Pathomechanismus von Dystonien sind. Unklar ist zudem, ob andere qMRTParameter, wie z.B. die T1-Zeit und die PD, bei Dystonien verändert sind.

\subsection{Fragestellung}

Gegenstand der Arbeit ist die Untersuchung mikrostruktureller Eigenschaften des Hirngewebes von Patienten mit fokaler idiopathischer Dystonie mit Methoden der qMRT (T1-, T2/T2*- und PD-Mapping). Anhand eines Vergleichs der gemessenen T1-, T2/T2*- und PD-Parameterkarten zwischen Patienten und gesunden Kontrollprobanden sollen erkrankungsbedingte Unterschiede in der Gewebemikrostruktur in vivo lokalisiert und in Zusammenhang mit den sehr heterogenen Ergebnissen vorheriger Studien gebracht werden. Speziell soll das Resultat einer rezenten MRT-Studie überprüft werden, gemäß der fokale idiopathische Dystonien mit einer erniedrigten T2*-Relaxationszeit im Globus pallidus assoziiert sind, die als Ausdruck eines erhöhten Eisengehaltes in diesem Kerngebiet interpretiert wurden ${ }^{91}$. Des Weiteren sollen bewegungsrelevante Areale (Basalganglien, Thalamus und Kleinhirn) auch hinsichtlich eventuell vorhandener Volumenänderungen untersucht werden. 


\section{Methodik}

\subsection{Studienteilnehmer}

\subsubsection{Patienten}

Es wurden 30 Patienten mit fokaler idiopathischer Dystonie mit Beginn im Erwachsenenalter in die Studie eingeschlossen. Diese wurden zum Zeitpunkt der Messung in der Bewegungsambulanz der Neurologie des Universitätsklinikums Frankfurt betreut und mit Botulinumtoxin A behandelt. Die Untersuchung fand im Brain Imaging Center (BIC) Frankfurt nach Ausschluss von Kontraindikationen statt. Dazu zählten Ausschlusskriterien für eine MRT wie beispielsweise das Tragen eines Herzschrittmachers oder Neurostimulators, Klaustrophobie und Schwangerschaft sowie weitere, relevante neurologische oder psychiatrische Erkrankungen (z.B. Schlaganfall, schwere Kopfverletzungen in der Vergangenheit, Schizophrenie). Die genauen Patientencharakteristika sind in Tabelle 2 dargestellt.

Tabelle 2: Patienten- und Probandencharakteristika im Vergleich

\begin{tabular}{|c|c|c|}
\hline Charakteristika & Patienten $(n=30)$ & Probanden $(n=30)$ \\
\hline \multicolumn{3}{|l|}{ Geschlecht } \\
\hline $\begin{array}{l}\text { weiblich } \\
\text { männlich }\end{array}$ & $\begin{array}{l}18(60 \%) \\
12(40 \%)\end{array}$ & $\begin{array}{l}18(60 \%) \\
12(40 \%)\end{array}$ \\
\hline \multicolumn{3}{|l|}{ Alter } \\
\hline $\begin{array}{l}\text { Mittelwert } \\
\text { Spannweite } \\
0 \text { - } 40 \text { Jahre } \\
41 \text { - } 50 \text { Jahre } \\
51 \text { - 60 Jahre } \\
\text { > 60 Jahre }\end{array}$ & $\begin{array}{l}52,3 \pm 13,45 \\
21-78 \\
4(13,3 \%) \\
9(30 \%) \\
8(26,7 \%) \\
9(30 \%)\end{array}$ & $\begin{array}{l}52,5 \pm 14,04 \\
22-80 \\
6(20 \%) \\
5(16,7 \%) \\
10(33,3 \%) \\
9(30 \%)\end{array}$ \\
\hline \multicolumn{3}{|l|}{ Dystonieform } \\
\hline $\begin{array}{l}\text { Zervikale Dystonie } \\
\text { Blepharospasmus } \\
\text { Schreibkrampf } \\
\text { Meige-Syndrom }\end{array}$ & $\begin{array}{l}20(66,7 \%) \\
5(16,7 \%) \\
3(10 \%) \\
2(6,7 \%)\end{array}$ & \\
\hline
\end{tabular}


Bei allen Patienten wurde je nach Erkrankungsbild der Schweregrad mittels einer etablierten Beurteilungsskala eingeschätzt. Für die zervikale Dystonie wurde die "Tsui Scale" und die "Toronto Western Spasmodic Torticollis Rating Scale" (TWSTRS) erhoben. Beide wurden bislang in vielen Studien zur spezifischen Einschätzung dieser Erkrankung eingesetz $\mathrm{t}^{93}$. Die "Tsui Scale“ beurteilt die Amplitude der anhaltenden Fehlstellung in allen drei Ebenen (Drehung, Seitwärtsneigung und Ante-/Retroflexion), die Dauer dieser, den Grad der Schulterelevation und den Schweregrad sowie Dauer des Tremors ${ }^{94}$. Die Skala ist in Tabelle 3 dargestellt. Aufgrund der schnellen Durchführbarkeit wird diese häufig in der klinischen Routine angewendet. Sie beachtet allerdings nicht den begleitenden Schmerz des Patienten, weshalb zusätzlich die TWSTRS erhoben worden ist. In die Berechnung des Scores fließen neben dem Schweregrad der Dystonie, auch die Einschränkung im alltäglichen Leben und der Schmerz ein ${ }^{95}$. Der Schweregrad wird in sechs Unterkategorien unterteilt: Grad der Kopffehlhaltung, Dauer, Effekt von sensorischen Tricks, Schulterhebung, Ausmaß der Beweglichkeit und Zeit, die der Patient den Kopf in Neutralstellung halten kann. Insgesamt können 35 Punkte vergeben werden, wobei die Dauer der Symptomatik doppelt gewertet wird. Die Leistungsskala beurteilt die Einschränkung des Patienten durch die zervikale Dystonie in den Bereichen Arbeit, Aktivitäten des täglichen Lebens, Autofahren, Lesen, Fernsehen und Aktivitäten außerhalb des Hauses. Jede Kategorie wird dabei mit null bis fünf Punkten bewertet. Es können maximal 30 Punkte erreicht werden. In die Schmerzskala fließen die Faktoren Schwere, Dauer und Behinderung durch den Schmerz ein. Die maximale Punktzahl beträgt 20. Insgesamt können in der TWSTRS bis zu 85 Punkte vergeben werden (siehe Anhang A.1). 
Tabelle 3: Tsui-Scale94

\begin{tabular}{|c|c|c|c|c|c|}
\hline \multicolumn{2}{|c|}{$\begin{array}{l}\text { Amplitude of sustained } \\
\text { movements }\end{array}$} & \multirow{2}{*}{$\begin{array}{c}\begin{array}{c}\text { Duration of } \\
\text { sustained } \\
\text { movements }\end{array} \\
1=\text { intermittend } \\
2=\text { constant }\end{array}$} & \multirow{2}{*}{$\begin{array}{l}\begin{array}{c}\text { Shoulder } \\
\text { elevation }\end{array} \\
0=\text { absent } \\
1=\text { mild and } \\
\text { intermittend } \\
2=\text { mild and } \\
\text { constant, or } \\
\text { severe and } \\
\text { intermittend } \\
3=\text { severe and } \\
\text { constant }\end{array}$} & \multicolumn{2}{|c|}{ Tremor } \\
\hline Rotation & $\begin{array}{l}0=\text { absent } \\
1=<15^{\circ} \\
2=15-30^{\circ} \\
3=>30^{\circ}\end{array}$ & & & Severity & $\begin{array}{l}1=\text { mild } \\
2=\text { severe }\end{array}$ \\
\hline Tilt & $\begin{array}{l}0=\text { absent } \\
1=<15^{\circ} \\
2=15-30^{\circ} \\
3=>30^{\circ}\end{array}$ & & & Duration & $\begin{array}{l}1= \\
\text { occasional } \\
2= \\
\text { continuous }\end{array}$ \\
\hline Ant/Retro & $\begin{array}{l}0=\text { absent } \\
1=\text { mild } \\
2=\text { moderate } \\
3=\text { severe }\end{array}$ & & & & \\
\hline $\begin{array}{l}A=\text { Rotatiol } \\
\text { Ant/Retro }\end{array}$ & + Tilt + & $B=1-2$ & $C=0-3$ & $\begin{array}{l}D=\text { Seve } \\
\text { Duration }\end{array}$ & $\begin{array}{l}(1-2) x \\
\text { 2) }\end{array}$ \\
\hline
\end{tabular}

Die Patienten mit Blepharospasmus sind mittels der "Jankovic Rating Scale“ (JRS) untersucht worden. Diese beurteilt den Schweregrad sowie die Frequenz der Symptome mit jeweils null bis vier Punkten, wobei vier die schwerste Form darstellt ${ }^{96}$. Es können dementsprechend maximal acht Punkte erreicht werden (siehe Tabelle 4).

Tabelle 4: Jankovic Rating Scale (JRS) ${ }^{97}$

\begin{tabular}{c|l|l}
\multicolumn{1}{|c}{ Schwere } & \multicolumn{1}{c}{ Frequenz } \\
\hline $\mathbf{0}$ & $\begin{array}{l}\text { keine } \\
\text { vermehrtes Blinzeln ausschließlich durch } \\
\text { externe Stimuli (helles Licht, Wind, Lesen } \\
\text { etc.) } \\
\text { mildes, aber spontanes Zucken der Au- } \\
\text { genlider (ohne tatsächlichen Spasmus), } \\
\text { nicht funktionell einschränkend } \\
\text { moderater Spasmus der Augenlider, } \\
\text { leicht einschränkend }\end{array}$ & $\begin{array}{l}\text { keine } \\
\text { leicht erhöhte Blinzelfrequenz } \\
\text { Dauer }\end{array}$ \\
$\mathbf{4}$ & $\begin{array}{l}\text { Schwerer, einschränkender Spasmus der Augenlider, <1s } \\
\text { Augenlider und evtl. weiterer Gesichts- } \\
\text { muskulatur }\end{array}$ & $\begin{array}{l}\text { Spasmus der Augenlider, >1s } \\
\text { Dauer, Augen >50\% der Wach- } \\
\text { zeit offen } \\
\text { funktionelle „Blindheit“ aufgrund } \\
\text { persistierender Spasmen, Augen } \\
>50 \% \text { der Wachzeit geschlossen }\end{array}$ \\
\hline
\end{tabular}


Die „Writer's Cramp Rating Scale” wurde zur Beurteilung der Patienten mit Schreibkrampf genutzt. Dabei werden die Handhaltung während des Schreibens sowie die Schreibgeschwindigkeit beurteilt (siehe Anhang A.2) ${ }^{98}$.

Für die Patienten mit Meige-Syndrom wurde die JRS und der „Oromandibular dystonia questionnaire“ (OMDQ-25) angewendet. Hierbei beantworten die Patienten 25 Fragen zur Beeinträchtigung durch die Erkrankung, psychosozialem Verhalten, Sprache und Schwierigkeiten beim Essen innerhalb der letzten zwei Wochen ${ }^{99}$. Es wird eine Beurteilung nach folgender Gewichtung vorgenommen: 0 = niemals, 1 = selten, 2 = manchmal, $3=$ oft, $4=$ immer. Folglich können bis zu 100 Punkte vergeben werden.

Des Weiteren wurden weitere allgemeine Patienteninformationen aufgenommen. Diese umfassten das Alter bei Krankheitseintritt, die Dauer der Erkrankung, Medikamenteneinnahme, weitere Erkrankungen, aktuelle Symptome, die Therapie (Botulinumtoxindosis), Abstand der MRT-Untersuchung zur letzten Botulinumtoxin-Gabe und aktuelle Wirkung der Therapie.

\subsubsection{Kontrollprobanden}

Es wurden 30 alters- und geschlechtsgematchte, gesunde Kontrollprobanden untersucht. Auch hier galten als Ausschlusskriterien Kontraindikationen gegen eine MRT (s.o.) sowie das Vorliegen von neurologischen oder psychiatrischen Erkrankungen. Die relevanten Probandencharakteristika sind im Vergleich zu den Patientencharakteristika in Tabelle 2 dargestellt.

Alle Studienteilnehmer wurden ausführlich mündlich und schriftlich über das Ziel der Studie, die geplanten Untersuchungsschritte, Risiken, ihre freiwillige Teilnahme an der Studie sowie über die datenschutzrechtlichen Folgen (elektronische oder schriftliche Speicherung der pseudonymisierten Daten, statistische Auswertung dieser, Veröffentlichung in wissenschaftlichen Artikeln) aufgeklärt. Insbesondere wurde auch auf den Umgang mit klinisch relevanten Zufallsbefunden hingewiesen. In diesem Falle wurden die Daten an einen qualifizierten 
medizinischen Experten (in der Regel einem Neurologen oder Neuroradiologen) weitergeleitet und der Studienteilnehmer benachrichtigt. Die Studie wurde von der Ethikkommission des Universitätsklinikums Frankfurt geprüft und zugelassen (Ethikvotum 233/16). Sie wurde entsprechend der aktuellen Deklaration von Helsinki (2013) durchgeführt.

\section{2 Überblick Datenerhebung und -auswertung}

Die MRT-Daten wurden nach folgendem Konzept erhoben, weiterverarbeitet und analysiert:

Schritt 1 An jedem Probanden wurden insgesamt sieben Gradienten-EchoDatensätze und vier Fast-Spin-Echo-Datensätze erhoben. Zudem erfolgten Messungen zur Bestimmung von B0 und des Sendespulenprofils.

Schritt 2 Aus den Rohdaten erfolgte eine Berechnung der korrigierten Parameterkarten für die T1, T2/T2* und PD. Des Weiteren wurde aus den Daten ein synthetischer magnetization-prepared rapid acquisition of gradient echoes (MP-RAGE) Datensatz errechnet.

Schritt 3 Die synthetischen MP-RAGE Bilder wurden mit dem Programm FreeSurfer 6.0.1 segmentiert und Volumina der subkortikalen Strukturen ausgelesen.

Schritt 4 Die Parameterkarten wurden auf den synthetischen MP-RAGE Datensatz koregistriert und in einen Standardraum transformiert.

Schritt 5 Zwecks Gruppenvergleich wurden die Parameterkarten einem voxelbasierten t-Test unterzogen und eine Korrektur für multiple Vergleiche angestellt. Das Ergebnis dieses Tests sind dreidimensionale Signifikanzkarten, die regionale Unterschiede anzeigen.

Schritt 6 Zusätzlich erfolgte eine sogenannte Region of Interest (ROI) basierte Analyse für pathophysiologisch relevante Hirnregionen.

Seite 21 


\subsection{Magnetresonanztomographie}

Die MRT-Messungen erfolgten mit einem 3-Tesla Ganzkörper MRT-Scanner (Siemens Medical Solutions, Erlangen) im Brain Imaging Center (BIC) Frankfurt. Zur Untersuchung wurde eine 8-Kanal-Kopfspule verwendet. Es kamen überwiegend im Haus entwickelte Messsequenzen zur Bestimmung der PD sowie der T1- und T2/T2*-Relaxationszeiten zum Einsatz. Das vollständige Sequenzprotokoll ist in Tabelle 5 angeführt. Die Gesamtlänge betrug 34:23 Minuten. Die Messung erfolgte im Schnitt zwei Wochen nach der letzten Injektion von Botulinumtoxin. In der Regel hat die Wirkung dann bereits nahezu ihr Maximum erreicht, wodurch Bewegungsartefakte vermindert werden sollten. 
Tabelle 5: Sequenzprotokoll

\begin{tabular}{|c|c|c|c|c|c|c|c|c|}
\hline Sequenz & Schnittebene & $\begin{array}{c}\text { räumliche } \\
\text { Auflösung } \\
\left(\mathrm{mm}^{3}\right)\end{array}$ & TR (ms) & TE (ms) & $\begin{array}{l}\text { Schicht- } \\
\text { dicke } \\
(\mathrm{mm})\end{array}$ & FoV $\left(\mathrm{mm}^{3}\right)$ & $\begin{array}{l}\text { Scan-Zeit } \\
\text { (min) }\end{array}$ & Absicht \\
\hline $\begin{array}{l}\text { zwei Gradienten- } \\
\text { Echo (Flip-Winkel } \\
4^{\circ} \text { und } 24^{\circ} \text { ) }\end{array}$ & sagittal & $1,0 \times 1,0 \times 1,0$ & 16,4 & 6,7 & 1 & $256 \times 224 \times 160$ & $9: 48$ & $\begin{array}{l}\text { VFA-Methode zur } \\
\text { Bestimmung von } \\
\text { T1 und PD }\end{array}$ \\
\hline Gradienten-Echo & sagittal & $4,0 \times 4,0 \times 4,0$ & 11 & 5 & 4 & $256 \times 224 \times 160$ & $0: 53$ & B1-Mapping \\
\hline Gradienten-Echo & sagittal & $2,0 \times 2,0 \times 2,0$ & 16,7 & 11 & 2 & $256 \times 224 \times 160$ & $2: 30$ & $\begin{array}{l}\text { T2*-Fehler (T1, } \\
\text { PD) }\end{array}$ \\
\hline Gradienten-Echo & transversal & $1,3 \times 1,3 \times 2,0$ & 60 & $10-52$ & 2 & $240 \times 180 \times 119$ & $5: 46$ & $\begin{array}{l}\text { T2*- und B0- } \\
\text { Mapping }\end{array}$ \\
\hline Gradienten-Echo & transversal & $2,5 \times 1,3 \times 2,0$ & 60 & $10-52$ & 2 & $240 \times 180 \times 119$ & 3:07 & $\begin{array}{l}\text { T2*- und B0- } \\
\text { Mapping }\end{array}$ \\
\hline Gradienten-Echo & transversal & $5,0 \times 1,3 \times 2,0$ & 60 & $10-52$ & 2 & $240 \times 180 \times 119$ & $1: 41$ & $\begin{array}{l}\text { T2*- und B0- } \\
\text { Mapping }\end{array}$ \\
\hline Fast-Spin-Echo & transversal & $1,3 \times 1,3 \times 2,0$ & 8000 & 103 & 2 & $240 \times 180 \times 119$ & $2: 02$ & T2-Mapping \\
\hline Fast-Spin-Echo & transversal & $1,3 \times 1,3 \times 2,0$ & 8000 & 120 & 2 & $240 \times 180 \times 119$ & $2: 02$ & T2-Mapping \\
\hline
\end{tabular}

$T R=$ Repetitionszeit (engl. repetition time), $T E=$ Echozeit (engl. echo time), Fo V = Field of View, VFA = variable flip angle 
Für die Erstellung der T1- und PD-Parameterkarten wurde die variable flip angle (VFA) Methode verwendet ${ }^{59}$. Hierbei werden unkorrigierte PD- und T1-Parameterkarten aus zwei Gradienten-Echo-Datensätzen, welche mit zwei unterschiedlichen Anregungswinkeln (flip angles) aufgenommen wurden, hergeleitet ${ }^{59}$. Die T1-Karten können anschließend aus den Kontrastunterschieden abgeleitet werden. In diesem Fall wurden die Anregungswinkel von $4^{\circ}$ (PD-Wichtung) und $24^{\circ}$ (T1-Wichtung) verwendet. Zur Verbesserung des Signal-Rausch-Verhältnisses und damit der Bildqualität wurde ein FLASH-EPI Hybrid-readout (fast low angle shot echo-planar imaging) angewendet ${ }^{100}$. Die Berechnung der korrigierten PDund T1-Karten ist in Kapitel 2.4 Auswertung dargestellt. U.a. ist eine Korrektur von B0-, B1- und T2*-Effekten notwendig. Hierzu wurde mithilfe von zwei mehrschichtigen Gradienten-Echo-Datensätzen Ungleichmäßigkeiten des B1 aufgezeichnet ${ }^{60}$. Zur Korrektur von Signalverlusten, die durch die T2*-Relaxation entstehen (T2*-Fehler), wurden zusätzlich zwei Gradienten-Echo-Datensätze mit unterschiedlicher Echozeit (TE, engl. echo time) aufgenommen.

Zur Messung der T2*-Relaxationszeit wurde eine multi-Gradienten-Echo-Sequenz mit acht Gradientenechos genutzt. Um Bewegungsartefakte, für die das T2*-Mapping besonders anfällig ist ${ }^{101}$, retrospektiv zu korrigieren, wurden insgesamt drei Datensätze mit verschiedenen räumlichen Auflösungen $(100 \% / 50 \% / 25 \%)$ aufgenommen ${ }^{102}$. Die erhobenen Daten erlauben ebenfalls das quantitative B0-Mapping. Dieses wird für die Korrektur von Inhomogenitäten des $\mathrm{B} 0$ benötigt.

Weiterhin wurden vier Fast-Spin-Echo-Datensätzen mit unterschiedlicher TE zur Erhebung der T2-Maps verwendet. Zusätzlich wurde ein hochauflösender, anatomischer MP-RAGE Datensatz für die Gewebesegmentierung errechnet (siehe Kapitel 2.4 Auswertung). 


\subsection{Auswertung}

Die Prozessierung und Auswertung der MRT-Daten wurde mit hauseigenen Programmen unter Verwendung von MatLab (MathWorks, Natick, MA), FSL 5.0.7 (FMRIB Software Library, Oxford, UK) und FreeSurfer 6.0.1 (Athinoula A. Martinos Center for Biomedical Imaging, Boston, MA) vorgenommen.

Das Präprozessieren, die Gewebesegmentierung und die Auswertung der MRTDaten orientierte sich an gängigen Standardprozeduren. Das Präprozessieren beinhaltete die Bearbeitung sowie Segmentierung der Datensätze mit FreeSurfer, die Koregistrierung auf die synthetischen MP-RAGE Datensätze und die Normalisierung in einen Standardraum. Anschließend erfolgte ein Voxel-basierter statistischer Gruppenvergleich der normalisierten Parameterkarten sowie eine statistische Auswertung der ROI-basierten MRT-Daten und der Volumina.

Zunächst erfolgte die Berechnung der korrigierten Parameterkarten. Die mittels der VFA-Methode erstellten T1-Parameterkarten wurden für B0- und B1-Effekte korrigiert ${ }^{60}$. Für das B0-Mapping wurden die erhobenen Daten mittels FSL „PRELUDE“ (Phase Region Expanding Labeler for Unwrapping Discrete Estimates) und FUGUE (FMRIB's Utility for Geometrically Unwarping EPIs) verarbeitet. Weiterhin wurden die T1-Maps für Effekte der residualen Quermagnetisierung verbessert ${ }^{103}$. Somit entstanden schließlich die korrigierten T1-Karten. Auch für die Errechnung der PD-Parameterkarten ist eine Berichtigung von Störfaktoren notwendig. Hierzu wurden Korrekturen für T2*-Effekte, Inhomogenitäten von B1 und des Empfangsspulen-Sensibilitätsprofils vorgenommen ${ }^{104}$.

Die T2/T2*-Relaxationszeiten wurden für jedes Voxel mittels exponentieller Anpassung aus den Fast-Spin-Echo-Datensätzen bzw. den Gradienten-Echo-Datensätzen mit den verschiedenen TE bestimmt.

Die synthetischen MP-RAGE Datensätze wurden aus den T1-Parameterkarten und so genannten Pseudo-PD-Maps berechnet ${ }^{105,}{ }^{106}$. Es entsteht ein synthetischer, PD/T1 gemischt-gewichteter Bilddatensatz. Für die Berechnung wurden folgende Parameter angenommen: Repetitionszeit $(\mathrm{TR}$, engl. repetition time $)=$ 
$1900 \mathrm{~ms}$, Inversionszeit (TI, engl. inversion time $)=900 \mathrm{~ms}$, Field of view $=256 \mathrm{x}$ $224 \times 160 \mathrm{~mm}^{3}$, Auflösung = $1 \times 1 \times 1 \mathrm{~mm}^{3}, \alpha=9^{\circ}$, Echointervall = 8,1 ms, 192 Phasenkodierungsschritte innerhalb der inneren Schleife mit symmetrischer $k$ space Abdeckung.

Diese synthetischen Bilder wurden mit dem Programm FreeSurfer 6.0.1 weiterverarbeitet. FreeSurfer ist eine frei erhältliche Software, die der Verarbeitung und dem Analysieren von MRT-Daten dient. Das Programm wurde zum Skullstripping, zur subkortikalen Segmentierung der Datensätze und zur Erhebung der Volumina genutzt. Zuerst erfolgte das Skullstripping, das der Entfernung nicht-zerebralen Gewebes wie beispielsweise des knöchernen Schädels, der Kopfhaut und der Dura aus Schädel-Bildern dient (brain-nonbrain segmentation). Die Segmentierung der Basalganglien, des Thalamus und des Cerebellums der MPRAGE Datensätze wurde mittels des „recon-all" Befehls der FreeSurfer „Toolbox" ${ }^{\prime 107}$ vorgenommen. Die Ergebnisse sind mit „Freeview" visuell kontrolliert worden. Es zeigten sich keine schwerwiegenden Fehlsegmentierungen, die einem manuellen Editieren bedurft hätten. Die aus der Segmentierung erhaltenen Masken wurden anschließend auf die quantitativen Parameterkarten koregistriert, um für jede ROI den durchschnittlichen Wert des jeweiligen Gewebeparameters zu berechnen (Mittelung über alle Bildvoxel einer ROI). Hierzu wurden zuerst die Masken der jeweils linken und rechten Hirnregion, z.B. des Putamen, in eine einzige Maske überführt. Zusätzlich zu den einzelnen Kerngebieten wurde durch Fusion eine „kombinierte“ Maske erstellt, welche die gesamte tiefe Graue Substanz beinhaltet. Voxel mit Werten von über $2000 \mathrm{~ms}$ in den T1-Masken, von über $200 \mathrm{~ms}$ in den T2-Masken und von über $100 \mathrm{~ms}$ in den T2*-Masken sind aus diesen ausgeschlossen worden, um eine systematische Verzerrung durch Partialvolumeneffekte an der Grenze zum Liquor cerebrospinalis zu minimieren.

Weiterhin wurde mittels FSL „FAST“ (FMRIB's Automated Segmentation Tool) ${ }^{108}$ eine Maske für die Weiße Substanz erstellt. Hierbei wurden Voxel, die mit Regionen der tiefen Grauen Substanz oder dem Kleinhirn überlappten, von der Analyse ausgeschlossen. Außerdem wurde die Maske mit einem Filter der Größe 3 x 3 x 
$3 \mathrm{~mm}^{3}$ erodiert, mit dem Ziel, ein Bias durch Partialvolumeneffekte mit der Grauen Substanz zu reduzieren.

Die Messung der Volumina wurde mithilfe von FreeSurfer vorgenommen. Die Volumenanalyse ermöglicht die Suche nach potentiellen Volumenabweichungen zwischen den Gruppen wie beispielsweise Atrophien oder Hemiatrophien.

Des Weiteren erfolgte eine Transformation der Bilddatensätze in einen Standardraum (Montreal Neurological Institute (MNI) 152 space), um identische anatomische Strukturen verschiedener Probandengehirne aufeinander abzubilden. Dies ist erforderlich, um Parameterkarten von verschiedenen Probanden voxelbasiert vergleichen zu können. Die Berechnung der Transformation erfolgte anhand der anatomischen MP-RAGE Datensätze, welche dann auf die anderen Parameterkarten übertragen wurde. Hierzu wurde FSL „FLIRT“ (FMRIB's Linear Image Registration Tool) und "FNIRT“ (FMRIB's Nonlinear Image Registration Tool) verwendet.

Die normalisierten Parameterkarten wurden einem ungepaarten t-Test unterzogen. Das Ergebnis ist eine Signifikanzkarte, anhand derer nach entsprechender Korrektur für multiple Vergleiche signifikante regionale Unterschiede zwischen dem Patienten- und Kontrollkollektiv abgelesen werden können (sog. „statistical parametric mapping"). Die Korrektur für multiple Vergleiche erfolgte permutationsbasiert mittels FSL „randomise“ 109 .

Neben der oben genannten voxelbasierten Analyse wurde auch eine ROI-basierte Analyse für folgende Hirnregionen durchgeführt: tiefe Graue Substanz, Weiße Substanz, Ncl. caudatus, Pallidum, Putamen, Thalamus sowie Kortex und Weiße Substanz des Cerebellums. Es erfolgte eine Mittelung der ROls über beide Hemisphären, um so die Variabilität der Messwerte zu reduzieren. Von einer seitengetrennten Beurteilung wurde auch aus dem Grund Abstand genommen, da es gerade bei Patienten mit zervikaler Dystonie oft schwierig ist, die komplexen und im Verlauf der Erkrankung auch seitenwechselnden Bewegungsmuster den Pathomechanismen einer bestimmten Hemisphäre zuzuordnen. 
Zusätzlich konnte in einer Untersuchung die bilaterale Repräsentation von Kopfbewegungen in den Basalganglien und dem Motorkortex nachgewiesen werden ${ }^{110}$. Somit ist die Kombination der ROIs beider Hemisphären legitimiert. Auch Aschermann et al. nutzten bereits den gleichen Ansatz ${ }^{91}$.

\subsection{Dokumentation und Statistik}

Zur Datenverarbeitung und statistischen Analyse der individuellen Probandendaten sowie der erhobenen quantitativen Parameter und Volumina wurden das Tabellenkalkulationsprogramm Microsoft Excel 2016 (Microsoft Corporation, Redmond, WA, USA) sowie die Statistiksoftware IBM SPSS Statistics 22.0.0 (IBM Corporation, Armonk, NY, USA) verwendet. Zur graphischen Darstellung der Ergebnisse wurde Sigma Plot 14.0 (SYSTAT Software, San Jose, CA, USA) genutzt. Weitere Abbildungen wurden mit MRIcron (University of Nottingham School of Psychology, Nottingham, UK) erzeugt und mit Photoshop CS4 11.0.2 (Adobe Systems, San Jose, CA, USA) nachbearbeitet.

Der Gruppenvergleich zwischen der Patienten- und Kontrollkohorte erfolgte mittels ungepaartem zweiseitigen t-Test (Mann-Whitney-U, SPSS Statistics). Die Zielvariablen waren dabei die T1- und T2/T2*-Relaxationszeiten, die PD sowie die Volumina der Basalganglien, des Thalamus, der Weißen Substanz und des Cerebellums. Weiterhin fand ein Vergleich der genannten Parameter für die Subgruppe der Patienten mit zervikaler Dystonie versus einer verkleinerten, altersund geschlechtsgematchten Kontrollgruppe statt. Ein $\mathrm{p}$-Wert von $<0,05$ wird als statistisch signifikant angenommen. Zusätzlich wurde auf eine signifikante Korrelation zwischen den Gewebeparametern (T1, T2/T2* und PD) der tiefen Grauen Substanz und dem Tsui-Score in der Subkohorte der Patienten mit zervikaler Dystonie getestet (Spearman-Korrelation, Sigma Plot). 


\section{Ergebnisse}

\subsection{Studienteilnehmer}

In die statistische Auswertung dieser Arbeit gingen die Daten von je 26 Probanden und Patienten mit fokal idiopathischer Dystonie ein. Jeweils vier Probanden und Patienten (drei Patienten mit zervikaler Dystonie, einer mit Blepharospasmus) mussten von den insgesamt 60 Studienteilnehmern aufgrund von Bewegungsartefakten in den MRT-Bildern aus der Studie ausgeschlossen werden. Auch nach Ausschluss dieser unterschied sich die Gruppe der Patienten und

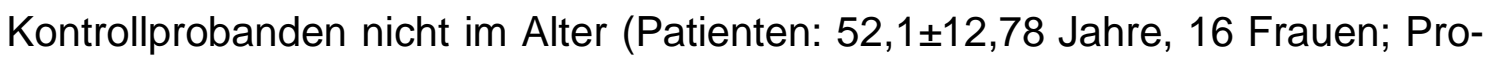
banden: $52,2 \pm 13,26$ Jahre, 16 Frauen; ungepaarter $t$-Test $p=0,91$ ).

Die Tabelle 6 zeigt die klinischen Charakteristika der eingeschlossenen Patienten $(\mathrm{n}=26)$. Insgesamt wurden 17 Patienten mit zervikaler Dystonie, vier mit Blepharospasmus, drei mit Schreibkrampf und zwei mit Meige-Syndrom in die Auswertung einbezogen. Die allgemeinen Patienteninformationen sind in Gruppen entsprechend des Krankheitsbildes ausgewertet worden. Außerdem sind die Ergebnisse der klinischen Beurteilungsskalen dargestellt. Vier Patienten sind neben den Injektionen von Botulinumtoxin A zusätzlich medikamentös mit Trihexyphenidyl (1 -6 mg) und einer mit Tizanidin ( $6 \mathrm{mg}$ ) behandelt worden. 
Tabelle 6: Klinische Daten

\begin{tabular}{|c|c|c|c|c|}
\hline & $\begin{array}{l}\text { zervikale } \\
\text { Dystonie }\end{array}$ & $\begin{array}{l}\text { Blepharo- } \\
\text { spasmus }\end{array}$ & $\begin{array}{l}\text { Schreib- } \\
\text { krampf }\end{array}$ & $\begin{array}{l}\text { Meige- } \\
\text { Syndrom }\end{array}$ \\
\hline $\begin{array}{l}\text { Manifestationsalter } \\
\text { (Jahre) }\end{array}$ & $39,8 \pm 11,94$ & $49,5 \pm 17,73$ & $36,7 \pm 19,63$ & $60,5 \pm 9,19$ \\
\hline $\begin{array}{l}\text { Erkrankungsdauer } \\
\text { (Jahre) }\end{array}$ & $11,2 \pm 12,33$ & $7,0 \pm 6,06$ & $5,7 \pm 2,31$ & $6,5 \pm 4,95$ \\
\hline $\begin{array}{l}\text { Ergebnis klinischer } \\
\text { Scores }\end{array}$ & $\begin{array}{r}5,2 \pm 2,70 \text { Tsui- } \\
\text { Scale/ } 24,9 \pm 10,72 \\
\text { TWSTRS }\end{array}$ & $\begin{array}{r}5,3 \pm 0,96 \\
J R S\end{array}$ & $\begin{array}{r}6,7 \pm 2,08 \\
\text { WCRS }\end{array}$ & $\begin{array}{r}5,0 \pm 4,24 \\
\text { JRS }\end{array}$ \\
\hline $\begin{array}{l}\text { durchschnittliche } \\
\text { Botulinumtoxin- } \\
\text { Dosis (IE) }\end{array}$ & $\begin{array}{l}1025 \text { Dysport/ } 215 \\
\text { Botox/ } 200 \text { Xeomin }\end{array}$ & $\begin{array}{l}94 \text { Botox/ } \\
68 \text { Xeomin }\end{array}$ & 25 Botox & 36 Botox \\
\hline $\begin{array}{l}\text { Abstand zur letzten } \\
\text { Injektion (Wochen) }\end{array}$ & $2,5 \pm 1,51$ & $1,3 \pm 1,32$ & $2,8 \pm 2,52$ & $2,8 \pm 1,06$ \\
\hline
\end{tabular}

Dargestellt sind der Mittelwert und die Standardabweichung der entsprechenden Werte. TWSTRS = Toronto Western Spasmodic Torticollis Rating Scale, JRS = Jankovic Rating Scale, $W C R S=$ Writer's Cramp Rating Scale, IE = Internationale Einheit.

\subsection{Visuelle Bildanalyse}

Bei der visuellen Durchsicht der MRT-Bilder konnte bei keinem der Patienten oder Probanden eine makroskopische, strukturelle Läsion oder relevante Atrophie festgestellt werden. Somit konnte bestätigt werden, dass nur Patienten mit fokaler Dystonie ohne nachweisbare anatomische Veränderungen in die Studie eingeschlossen wurden.

\subsection{Resultate der Gewebesegmentierung}

Die mithilfe von FreeSurfer vorgenommene Gewebesegmentierung ist exemplarisch für einen Probanden in Abbildung 3 dargestellt. 


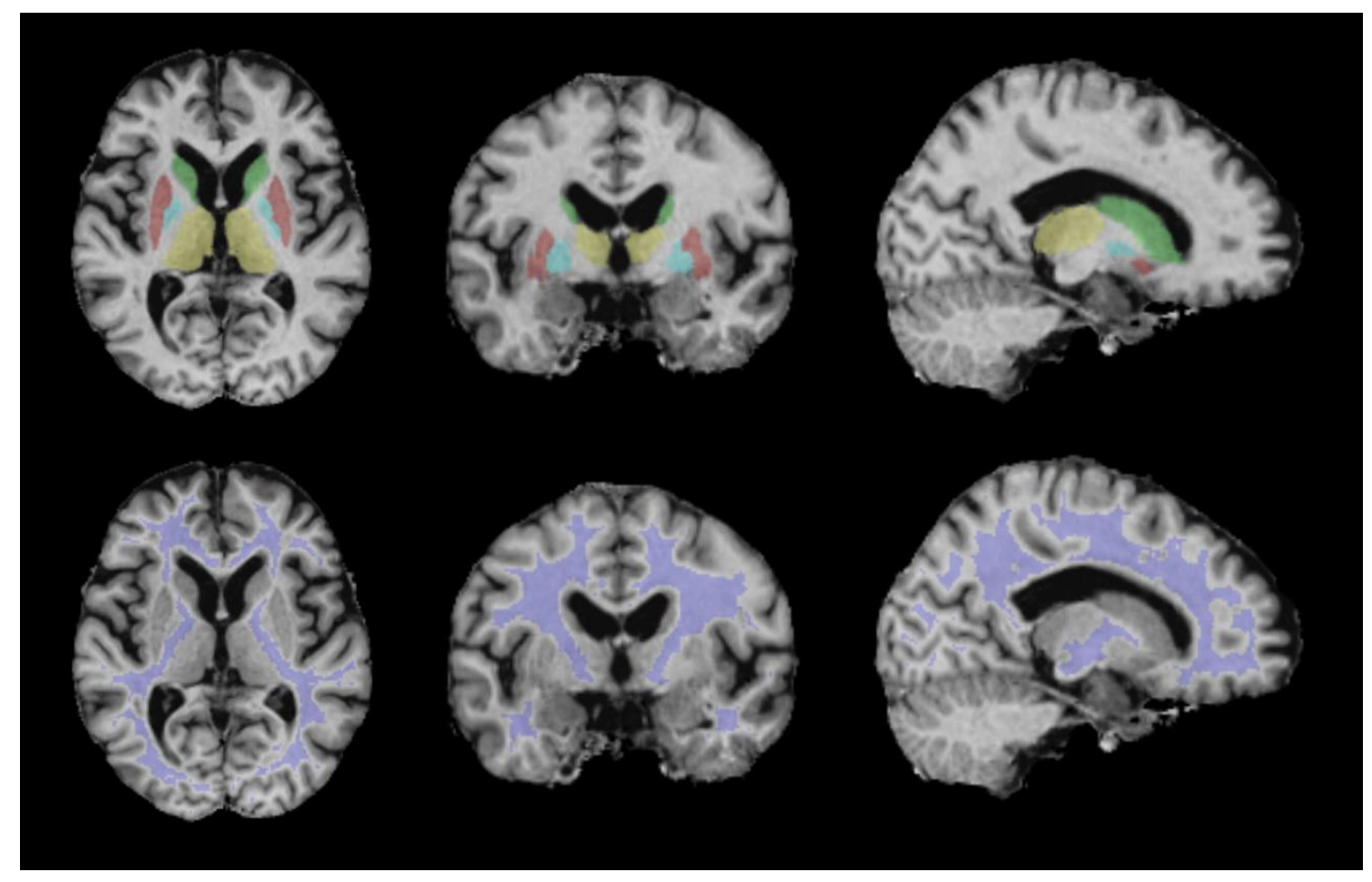

Abbildung 3: Gewebesegmentierung der tiefen Grauen und Weißen Substanz

Im oberen Bereich ist die Segmentierung der Basalganglien und des Thalamus in drei orthogonalen Schnittebenen dargestellt (grün: Nucleus caudatus, rot: Putamen, Cyan: Pallidum, gelb: Thalamus). Der untere Bereich zeigt die Segmentierung der Weißen Substanz (blau). Darstellung im Montreal Neurological Institute (MNI) 152 standard space, Schnittebenen bei $x=16 \mathrm{~mm}, y=-9 \mathrm{~mm}, z=8 \mathrm{~mm}$.

\subsection{Voxel-weise Bildanalyse}

Im Voxel-weisen Vergleich der qMRT-Parameterkarten mittels ungepaartem tTest ergaben sich nach Korrektur für multiple Vergleiche keine statistisch signifikanten Gruppenunterschiede für alle vier untersuchten Gewebeparameter (T1, $\mathrm{T} 2 / \mathrm{T}^{*}$ und $\mathrm{PD}$ ). Die unkorrigierten statistischen Karten (t-Maps) für den Gruppenvergleich von Patienten und Kontrollprobanden bezüglich der T1-Relaxationszeit und PD sind exemplarisch für eine axiale Schicht in Abbildung 5 dargestellt. Die errechneten quantitativen Parameterkarten werden für einen repräsentativen Probanden beispielhaft in Abbildung 4 gezeigt. 


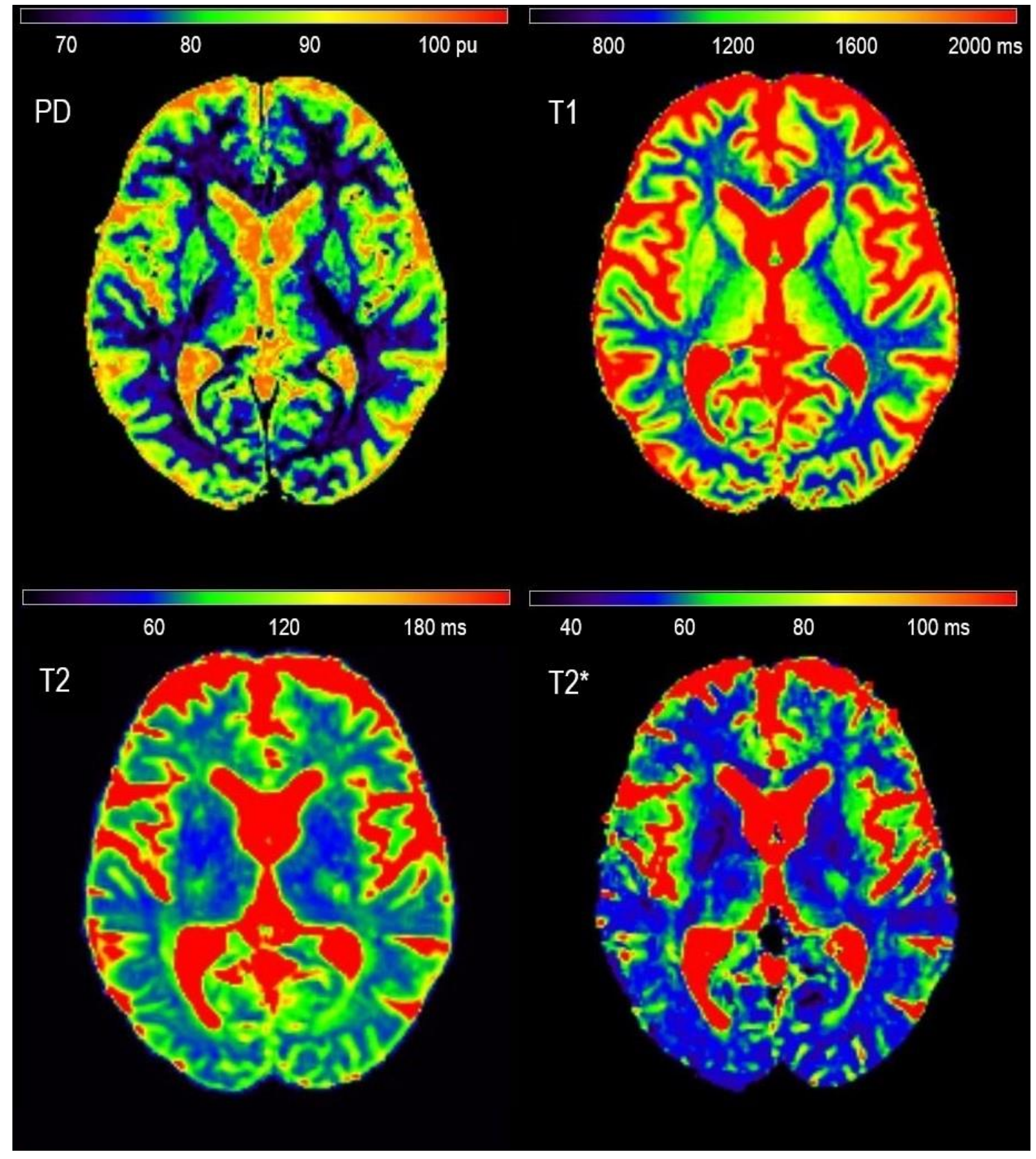

Abbildung 4: Quantitative T1-, T2/T2*- und PD-Karten

Darstellung der errechneten quantitativen Gewebeparameterkarten im Montreal Neurological Institute (MNI) 152 standard space (axiale Schnittebene $z=8 \mathrm{~mm}$ ). $P D=$ Protonendichte. 


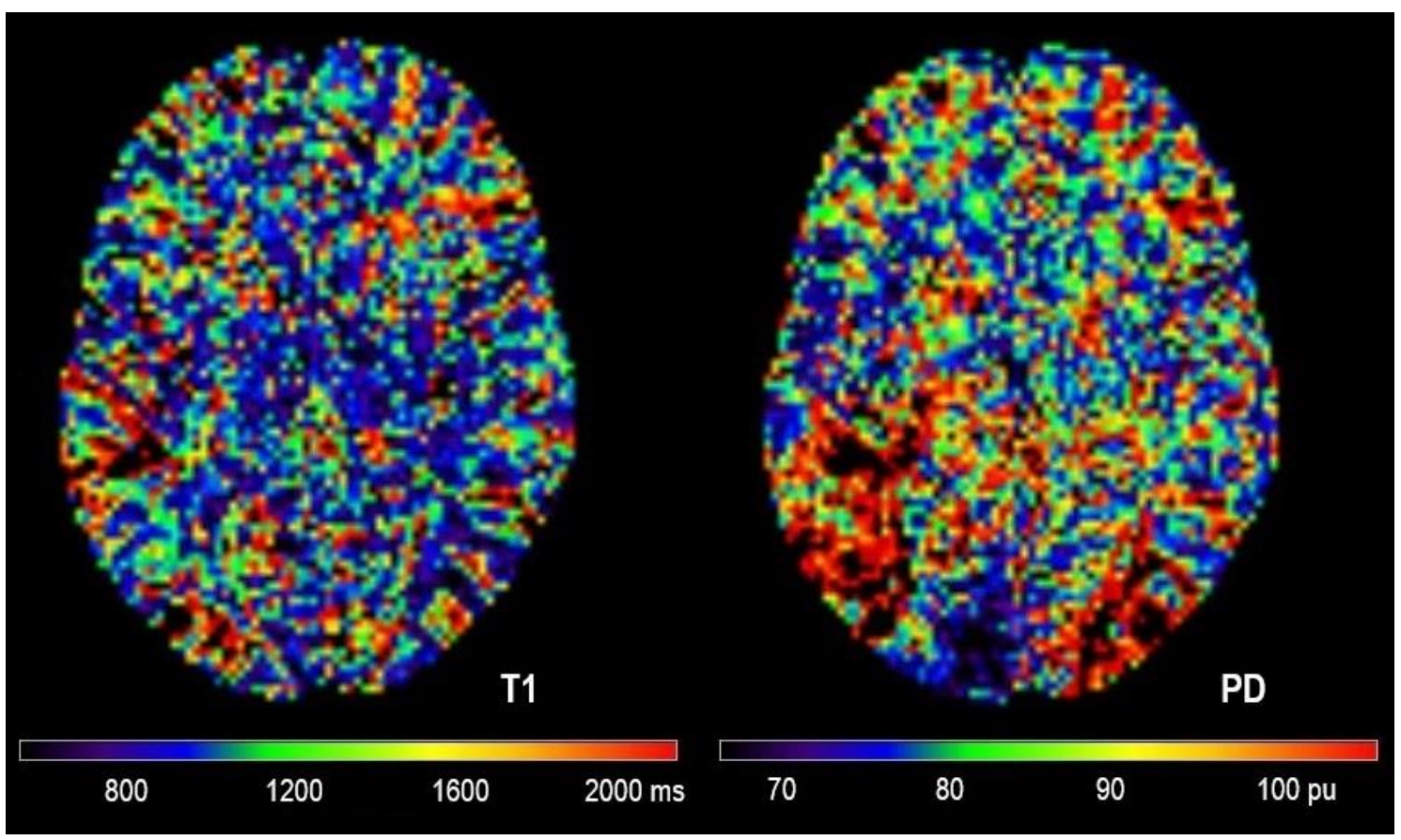

Abbildung 5: Unkorrigierter voxelbasierter t-Test für den T1- und PD-Gruppenvergleich

Exemplarische Darstellung der unkorrigierten statistischen Karten (t-Maps) für den Gruppenvergleich von Patienten und Probanden für eine axiale Schicht im Montreal Neurological Institute (MNI) 152 standard space (Schnittebene $z=8 \mathrm{~mm}$ ). $P D=$ Protonendichte.

\subsection{Region of Interest basierte Analyse}

Auch die ROI-basierte Analyse ergab keine signifikanten Gruppenunterschiede hinsichtlich sämtlicher untersuchter MRT-Parameter (T1, T2/T2* und PD) sowie Regionen. Es fand sich lediglich eine Tendenz zu erhöhten T1-Relaxationszeiten bei Patienten mit Dystonie im Bereich der gesamten tiefen Grauen Substanz ( $p$ $=0,07)$, des Ncl. Caudatus $(p=0,07)$, des Putamens $(p=0,09)$ und des Thalamus ( $p=0,05$; Mann-Whitney-U-Test). Für das Pallidum, die Weiße Substanz und den Kortex sowie die Weiße Substanz des Cerebellums lagen die $p$-Werte für den T1-Gruppenvergleich deutlich weiter von der Signifikanzgrenze entfernt (Pallidum $p=0,16$, Weiße Substanz $p=0,13$, Cerebellum Kortex $p=0,53$, Cerebellum Weiße Substanz $p=0,11)$. Die Ergebnisse für alle Parameter und 
Regionen sind in den Abbildungen 6.1 bis 6.4 in Form von Boxplots dargestellt. Die minimalen p-Werte für die anderen Parameter betrugen 0,46 (T2), 0,26 (T2*) und 0,29 (PD). Bei der Betrachtung der PD fällt eine etwas größere Spannweite und Standardabweichung in der Gruppe der Dystonie-Patienten auf.

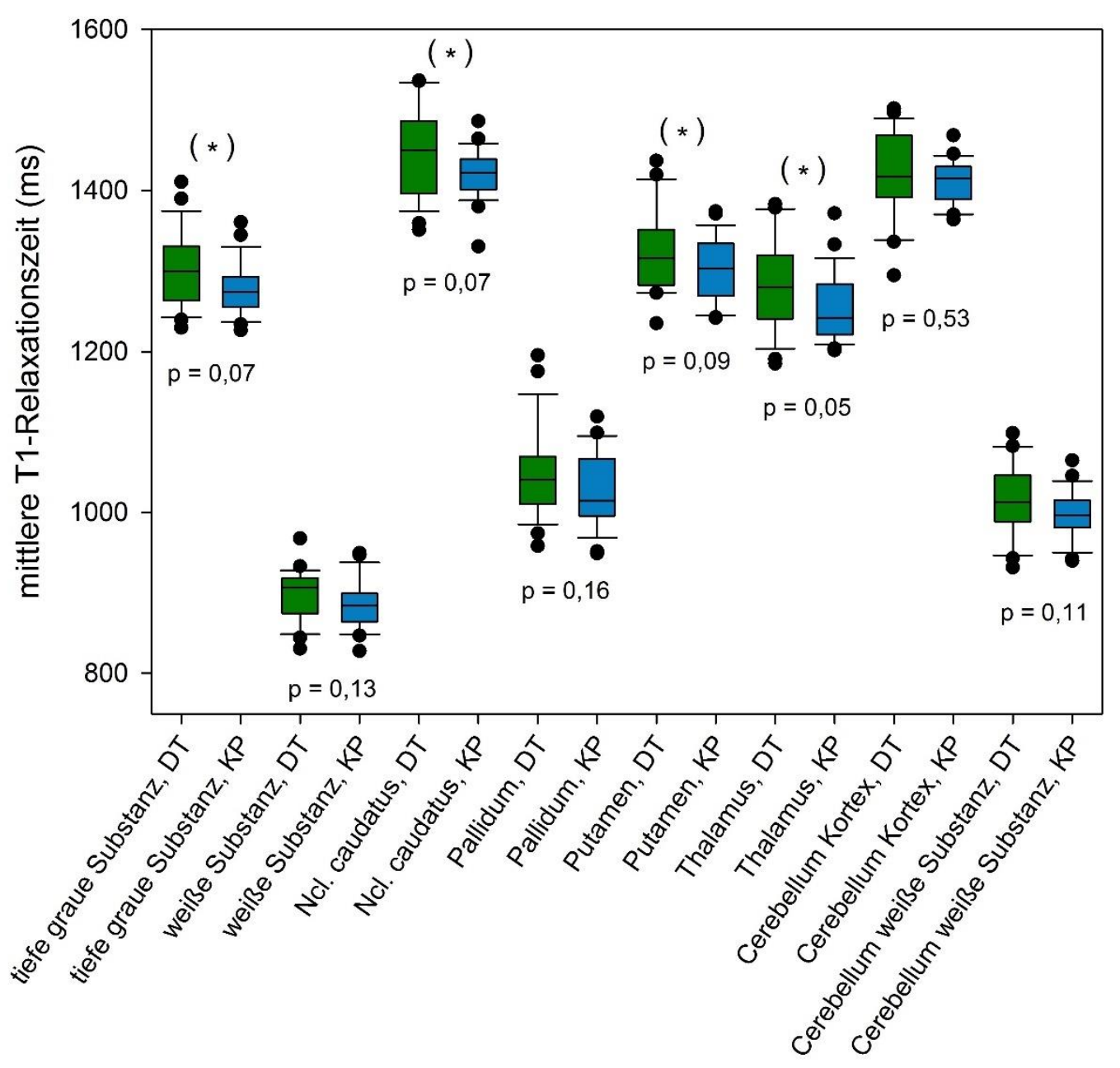

\section{Abbildung 6.1: Ergebnisse der Messungen der T1-Relaxationszeit}

Ergebnisse der Region of Interest (ROI) basierten Analyse für die T1-Relaxationszeit. Für beide Gruppen werden der Median, das 25\%- und 75\%-Quartil, das 90\%-Konfidenzintervall sowie Ausreißer gezeigt. (*) markiert ROIs, für die der Gruppenvergleich eine statistische Tendenz ergab ( $p$-Wert zwischen 0,05 und 0,1). DT = Dystoniepatienten, KP $=$ Kontrollprobanden, $\mathrm{Ncl} .=$ Nucleus . 


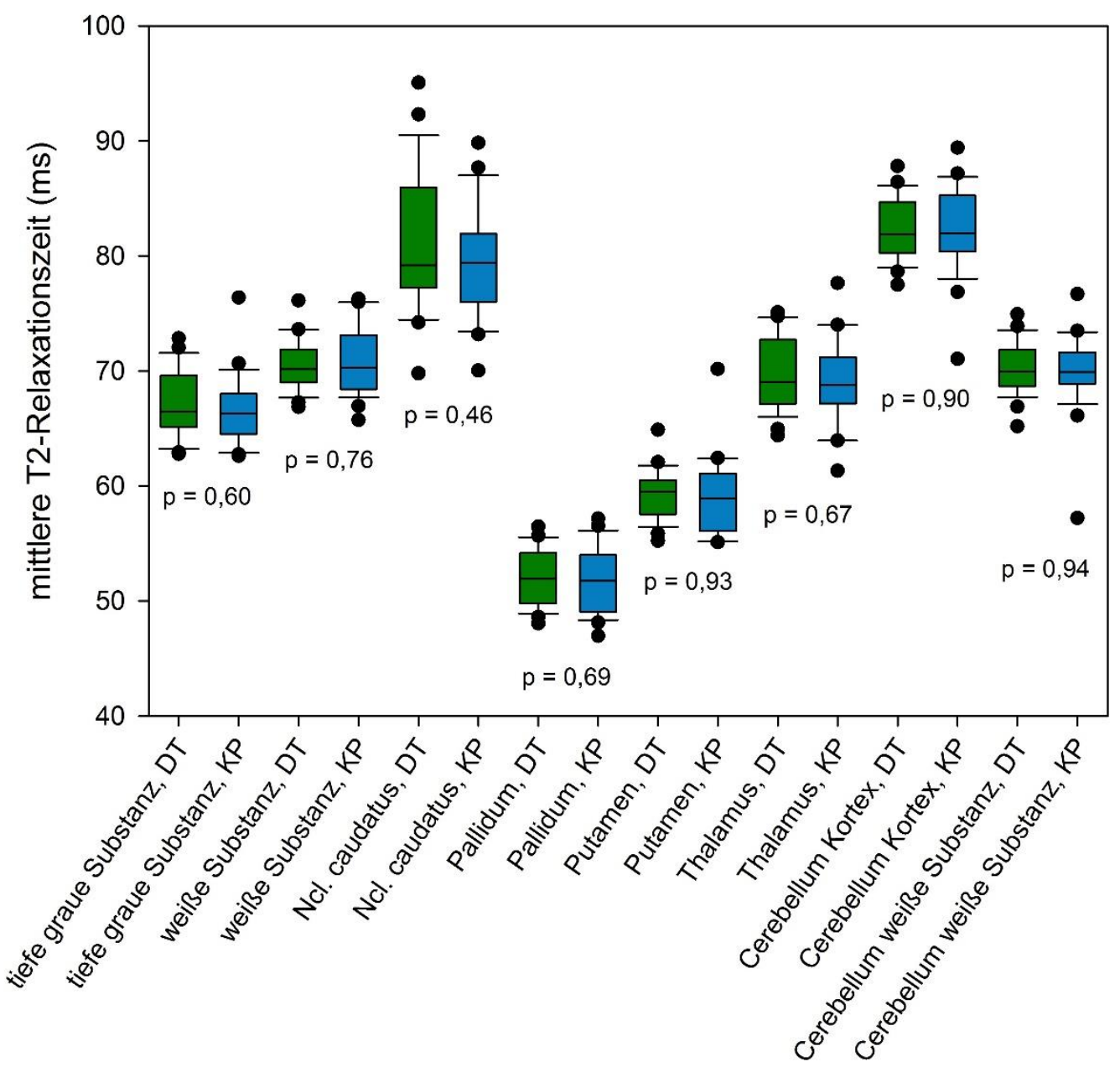

Abbildung 6.2: Ergebnisse der Messungen der T2-Relaxationszeit

Ergebnisse der Region of Interest (ROI) basierten Analyse für die T2-Relaxationszeit. Für beide Gruppen werden der Median, das 25\%- und 75\%-Quartil, das 90\%-Konfidenzintervall sowie Ausreißer gezeigt. $D T=$ Dystoniepatienten, $K P=$ Kontrollprobanden, $\mathrm{Ncl}$. = Nucleus. 


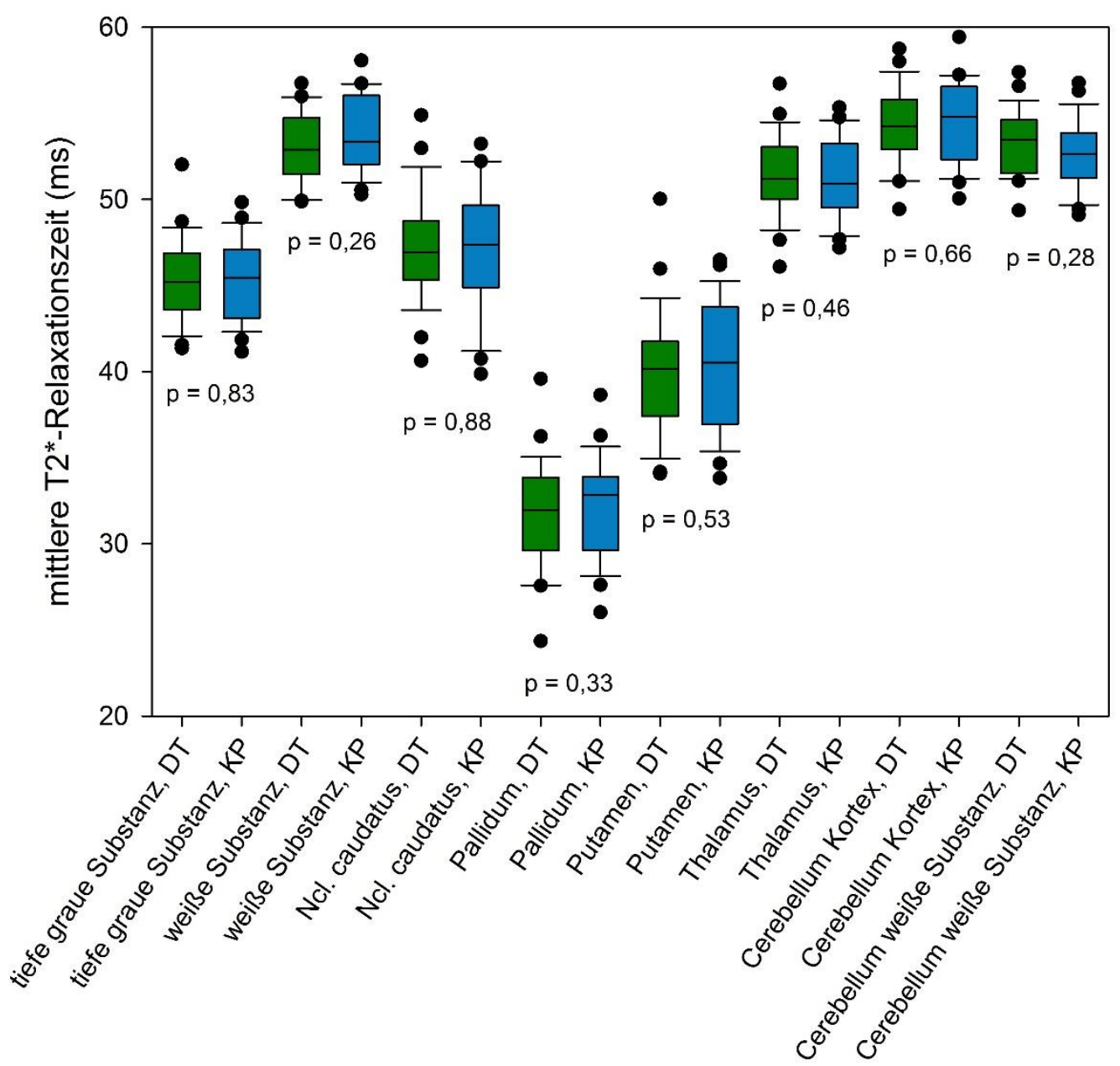

Abbildung 6.3: Ergebnisse der Messungen der T2*-Relaxationszeit

Ergebnisse der Region of Interest (ROI) basierten Analyse für die T2*-Relaxationszeit. Für beide Gruppen werden der Median, das 25\%- und 75\%-Quartil, das 90\%-Konfidenzintervall sowie Ausreißer gezeigt. $D T=$ Dystoniepatienten, $K P=$ Kontrollprobanden, $N c l$. = Nucleus. 


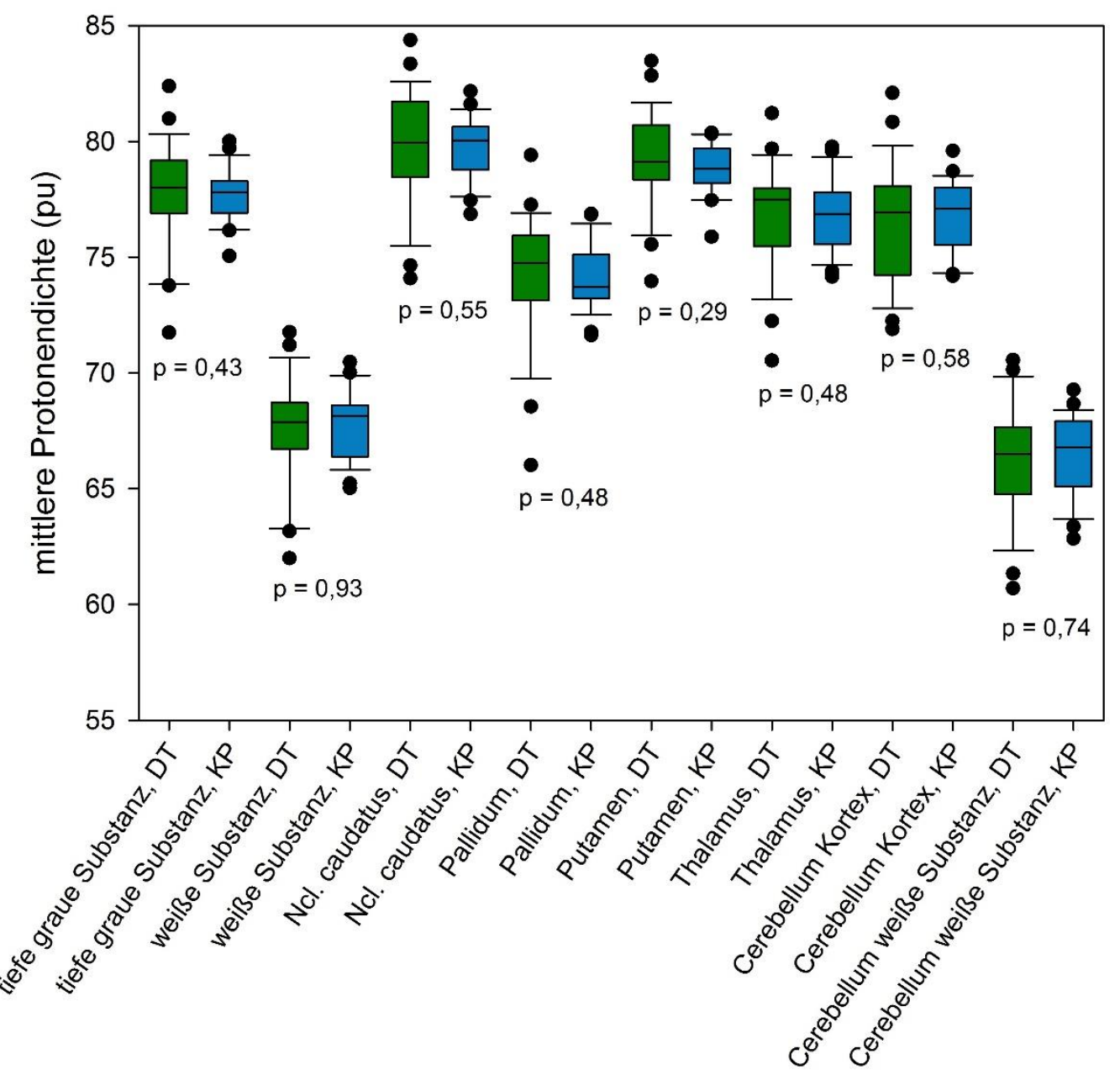

Abbildung 6.4: Ergebnisse der Messungen der Protonendichte

Ergebnisse der Region of Interest (ROI) basierten Analyse für die Protonendichte. Für beide Gruppen werden der Median, das 25\%- und 75\%-Quartil, das 90\%-Konfidenzintervall sowie Ausreißer gezeigt. $D T=$ Dystoniepatienten, $K P=$ Kontrollprobanden, $\mathrm{Ncl}$. = Nucleus.

\subsection{Volumenanalyse}

Die Ergebnisse der Volumenanalyse sind in Abbildung 7 in Form von Boxplots dargestellt. Auch hier ergaben sich keine signifikanten Gruppenunterschiede ( $p$ $\geq 0,31$ für alle ROIs). Insbesondere ergab sich keine Abweichung des Volumens 
der subkortikalen Grauen Substanz (Dystoniegruppe: $53.800 \pm 5.700 \mathrm{~mm}^{3}$; Kontrollgruppe: $54.600 \pm 5.070 \mathrm{~mm}^{3}$ ). Der $p$-Wert im Mann-Whitney-U-Test lag in dieser Region bei 0,58.

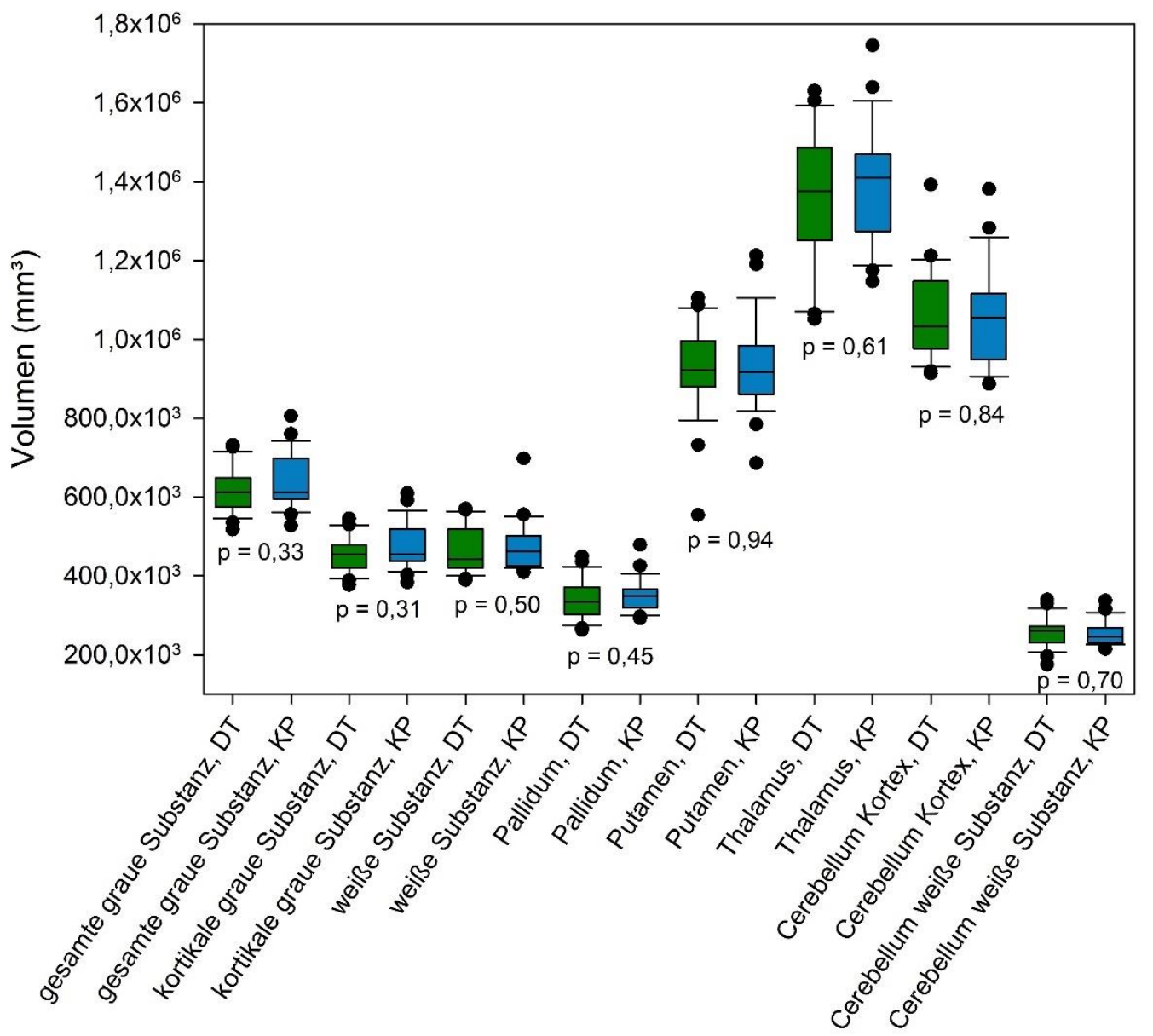

Abbildung 7: Ergebnisse der Volumenanalyse für verschiedene Regions of Interest

Für beide Gruppen werden der Median, das 25\%- und 75\%-Quartil, das 90\%-Konfidenzintervall sowie Ausreißer gezeigt. Das Volumen des Putamen, Pallidums und Thalamus wurde zur besseren Darstellung verhundertfacht, das Volumen des Cerebellums verzehnfacht. $D T=$ Dystoniepatienten, $K P=$ Kontrollprobanden . 


\subsection{Subgruppenanalyse}

Die erfolgte Subgruppenanalyse der 17 Patienten mit zervikaler Dystonie (neun Frauen, 51,0 \pm 8,88 Jahre) und einer alters- und geschlechtsgematchten Kontrollkohorte (neun Frauen, 50,6 \pm 9,30 Jahre) erbrachte ebenfalls keine signifikanten Unterschiede für sämtliche untersuchten qMRT-Parameter (T1, T2/T2* und PD). Dies gilt sowohl für den Voxel-weisen Vergleich der Parameterkarten als auch für die ROI-basierten Analysen. Auch ergaben sich keine signifikanten Gruppenunterschiede hinsichtlich der ROI-Volumina ( $p \geq 0,21$, Abbildung 9). Es konnte lediglich eine Tendenz zu erhöhten T1-Relaxationszeiten bei Patienten mit Dystonie im Bereich der Weißen Substanz $(p=0,05)$ und des Thalamus $(p=$ 0,09 ) festgestellt werden. Weiterhin ergab sich eine Tendenz zu erniedrigten $T 2^{*}-$ Relaxationszeiten im Bereich des Ncl. caudatus ( $p=0,09$; Mann-Whitney-UTest). Die p-Werte der weiteren MRT-Parameter lagen bei über 0,25 (PD) und über 0,68 (T2). Die Resultate der ROI-basierten Analyse sind in Abbildung 8 in Form von Boxplots dargestellt. Es fällt ebenso wie in der Analyse der gesamten Dystonie-Kohorte eine erhöhte Spannweite und Standardabweichung der PD in der Dystoniegruppe auf. 

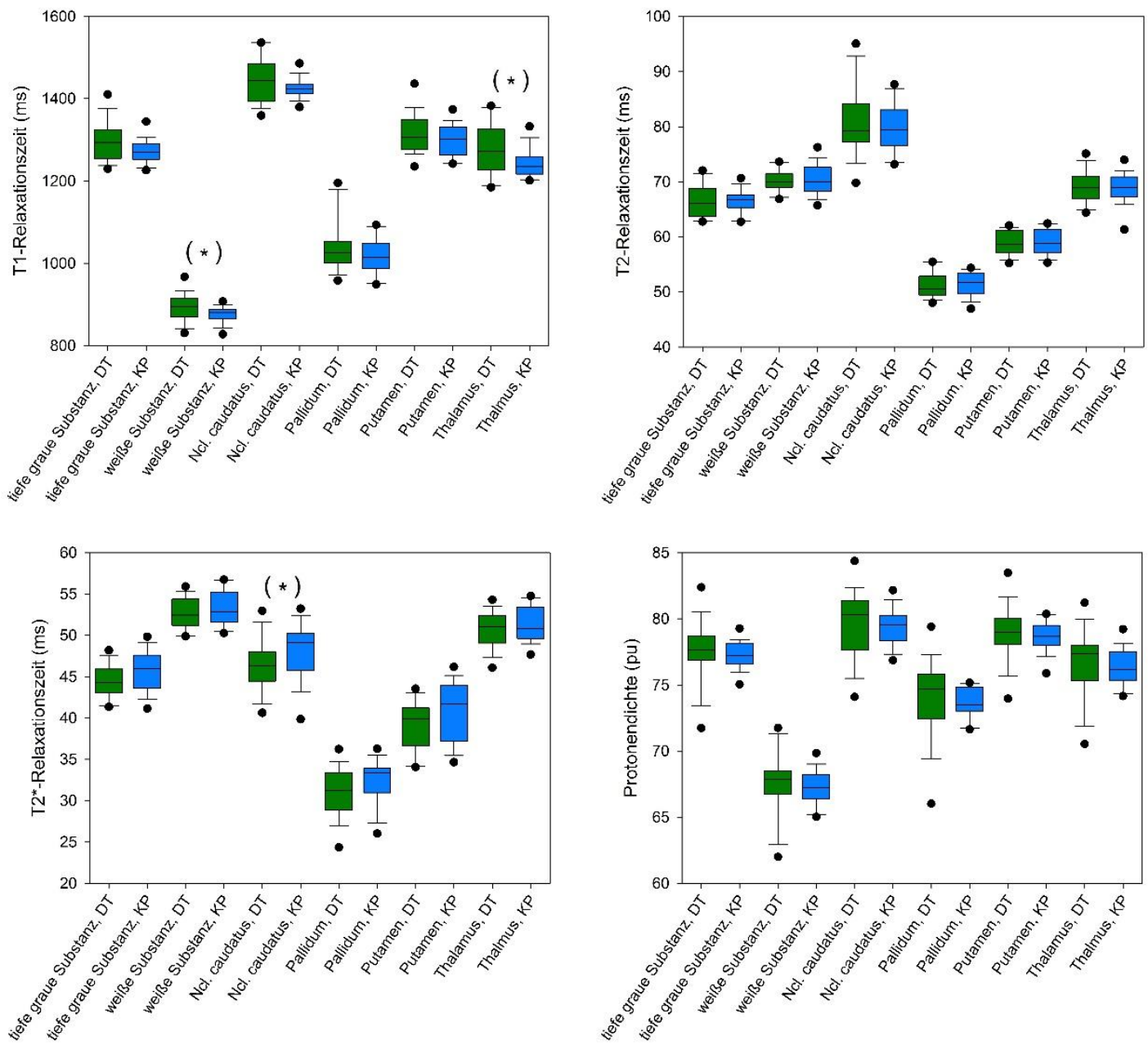

Abbildung 8: Ergebnisse der Subgruppenanalyse für Patienten mit zervikaler Dystonie

Ergebnisse der Region of Interest (ROI) basierten Analyse für die quantitativen Gewebeparameter (T1, T2/T2* und Protonendichte). Für beide Gruppen werden der Median, das 25\%-und 75\%-Quartil, das 90\%-Konfidenzintervall sowie Ausreißer gezeigt. (*) markiert ROls, für die der Gruppenvergleich eine statistische Tendenz ergab ( $p$-Wert zwischen 0,05 und 0,1). $D T=$ Dystoniepatienten, $K P=$ Kontrollprobanden, $N c l .=$ Nucleus. 


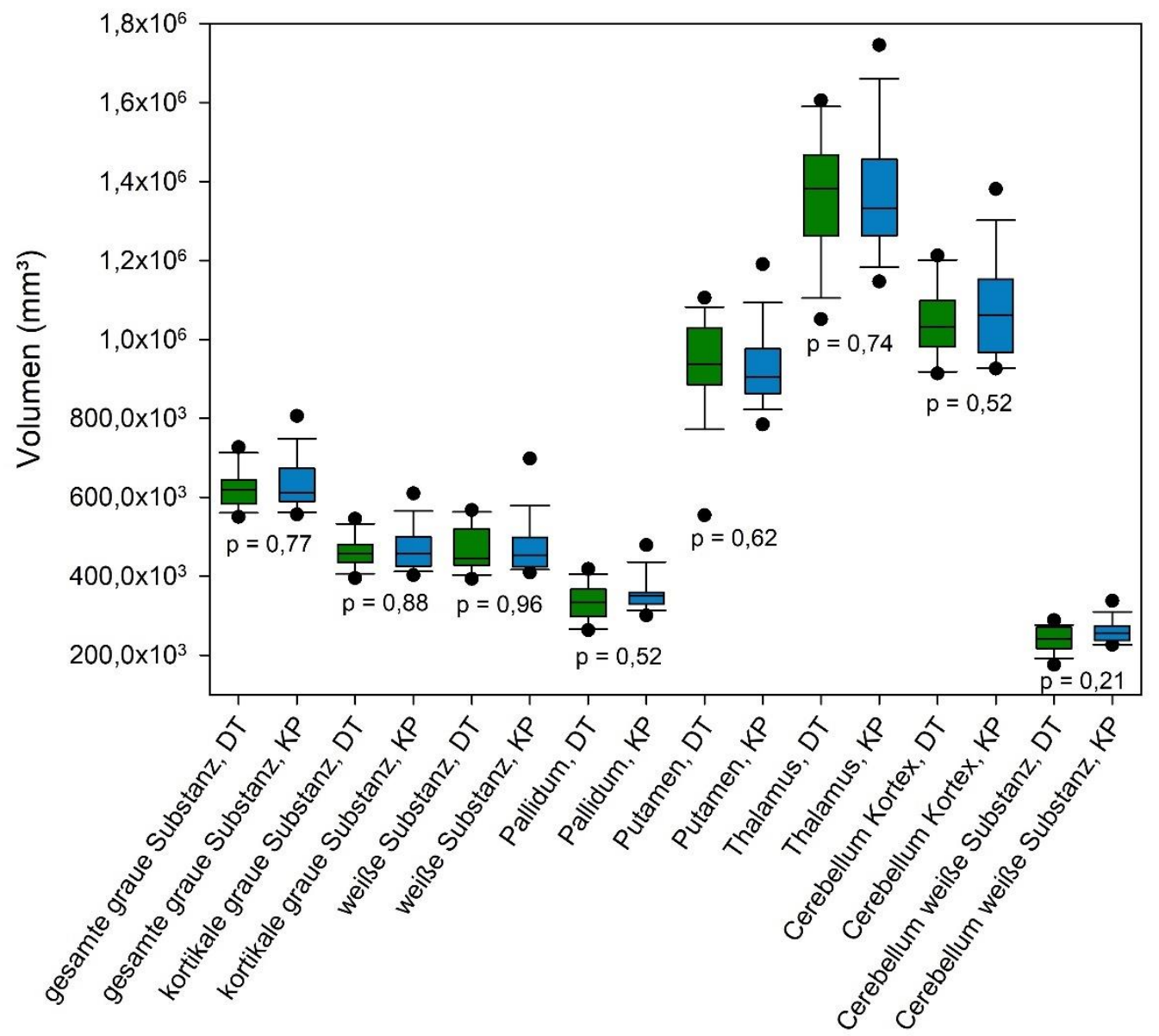

Abbildung 9: Ergebnisse der Volumenanalyse für Patienten mit zervikaler Dystonie

Für beide Gruppen werden der Median, das 25\%- und 75\%-Quartil, das 90\%-Konfidenzintervall sowie Ausreißer gezeigt. Das Volumen des Putamen, Pallidums und Thalamus wurde zur besseren Darstellung verhundertfacht, das Volumen des Cerebellums verzehnfacht. $D T=$ Dystoniepatienten, $K P=$ Kontrollprobanden . 
Die Korrelationsanalyse zwischen den Gewebeparametern (T1, T2/T2* und PD) der tiefen Grauen Substanz und dem Tsui-Score ergab keine signifikante Korrelation $(-0,20 \leq r \geq 0,19, p \geq 0,43$ für alle qMRT-Parameter, Spearman Rang Korrelationen). Ein Streudiagramm inklusive Regressionsgeraden ist beispielhaft für die T1-Relaxationszeiten in Abbildung 10 gezeigt.

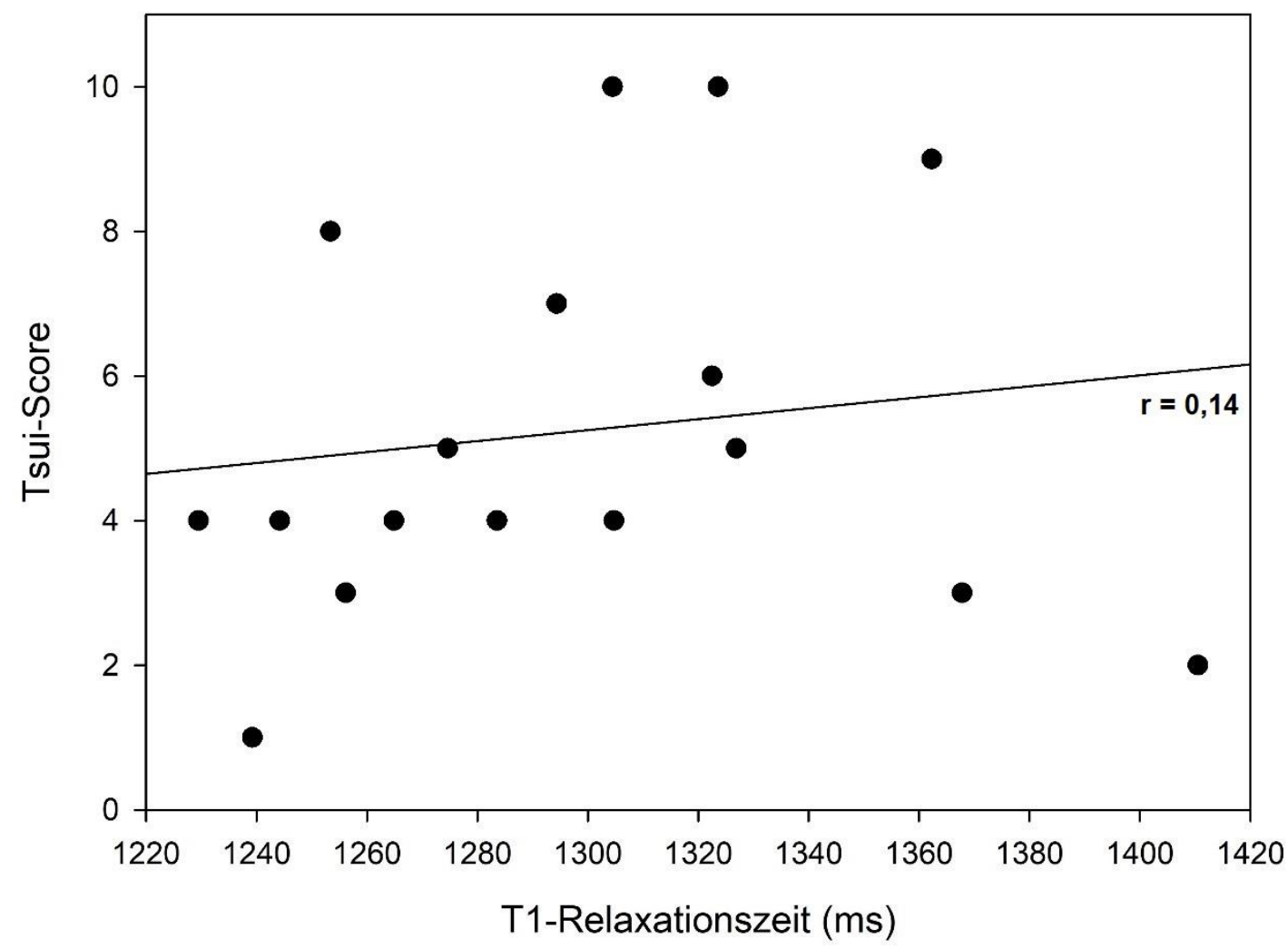

Abbildung 10: Streudiagramm von T1-Relaxationszeiten versus Tsui-Scores

Tsui-Scores und T1-Relaxationszeiten der tiefen Grauen Substanz bei Patienten mit zervikaler Dystonie waren nicht korreliert $(r=0,14, p=0,43)$. 


\section{Diskussion}

Zusammenfassend konnten weder in der Voxel-weisen noch in der ROI-basierten Analyse signifikante Veränderungen der T1- und T2/T2*-Relaxationszeiten oder der PD bei Patienten mit fokaler idiopathischer Dystonie im Vergleich zu einer gesunden Kontrollkohorte nachgewiesen werden. Des Weiteren ergaben sich keine statistischen Hinweise auf Gruppenunterschiede in den Volumina des zerebralen Kortex, der Weißen Substanz, der Basalganglienkerngebiete und des Cerebellums. Gleichfalls negative Ergebnisse zeigten sich in den Subgruppenanalysen bezüglich des Vergleichs von Patienten mit zervikaler Dystonie und gesunden Kontrollpersonen. Ferner konnte kein korrelativer Zusammenhang zwischen dem klinischen Schweregrad der zervikalen Dystonie und der Gewebeparameter nachgewiesen werden. Somit ergaben sich entgegen der ursprünglichen Hypothese keine qMRT-basierten Hinweise auf krankheitsspezifische mikrostrukturelle Gewebeveränderungen bei Patienten mit idiopathischer Dystonie.

\subsection{Quantitative Gewebeparameter}

\subsubsection{T2*-Relaxationszeit}

Im Widerspruch zu den Ergebnissen von Aschermann et al. ${ }^{91}$ ergab sich in dieser Untersuchung kein Hinweis auf erhöhte Eisenablagerungen im Bereich der Basalganglien bei fokalen idiopathischen Dystonien. Die T2*-Relaxationszeit ist ein sensitiver Marker für Eisenablagerungen in der Grauen Substanz ${ }^{111}$. Es konnte lediglich eine Tendenz zu erniedrigten T2*-Zeiten im Bereich des Ncl. caudatus in der Subkohorte der Patienten mit zervikaler Dystonie gefunden werden. Aschermann et al. hingegen konnten in letztgenannter qMRT-Studie erhöhte $\mathrm{R} 2^{*}$-Werte (also erniedrigte $\mathrm{T}^{*}$-Werte) im Globus pallidus, nicht aber im Ncl. caudatus bei Patienten mit zervikaler Dystonie nachweisen. Das Ergebnis wurde als Hinweis auf erhöhte Eisenablagerungen interpretiert. Die Kohorten mit jeweils $n=12$ waren etwas kleiner als in der vorliegenden Studie $(n=26$ bzw. $n=17)$. Ferner waren die Patienten und Probanden durchschnittlich jünger (Patienten im 
Durchschnitt 6,9 Jahre, Probanden 7,5 Jahre). Die demographischen Daten weichen allerdings nicht genug ab, um die Differenzen der Ergebnisse zu begründen. Weiterhin sind die Techniken der Datenaufnahme und -verarbeitung gleichartig. So nutzen Aschermann et al. zum R2*-Mapping zwölf Gradientenechos ( $\mathrm{TE}_{1}=3,58 \mathrm{~ms}$ ), 104 axiale Schichten und eine Schichtdicke von $1 \mathrm{~mm}$. In dieser Untersuchung wurden acht Gradientenechos ( $\mathrm{TE}_{1}=10 \mathrm{~ms}$ ), 120 axiale Schichten in drei Sequenzen und eine Schichtdicke von $2 \mathrm{~mm}$ verwendet. Somit wurden in beiden Arbeiten vergleichbar qualitativ hochwertige Datensätze erhoben. Die abweichenden Resultate können gegebenenfalls durch die Nutzung von automatischen Segmentierungsmethoden entstehen. So können moderate Unterschiede in den Algorithmen und Trainingsdatensätzen die Ergebnisse verändern. Zudem sollte berücksichtigt werden, dass die T2* ${ }^{*}$ Zeit ebenfalls durch andere Faktoren als den Eisengehalt beeinflusst werden kann. Beispiele dafür sind vaskuläre oder mikrostrukturelle Veränderungen, aber auch weitere Minerale wie Calcium oder Kupfer $^{112}$. Somit können diese Faktoren den direkten Rückschluss auf den Eisengehalt verzerren. Ferner sind T2*-Sequenzen sehr bewegungsanfällig, sodass hier eine speziell in diesem Haus entwickelte Methode zur Post-hoc Bewegungskorrektur angewendet wurde. Dies stellt eine mögliche Ursache der unterschiedlichen Studienergebnisse dar. Insgesamt weisen die Ergebnisse der vorliegenden Arbeit nicht auf einen erhöhten Eisengehalt in verschiedenen Regionen motorischer Kontrolle bei idiopathischer Dystonie hin.

\subsubsection{T2-Relaxationszeit}

Weiterhin konnten keine Veränderungen der T2-Relaxationszeit nachgewiesen werden. Die T2-Zeit wird v.a. durch den Grad der Myelinisierung sowie den Gehalt an Wasser und Eisen beeinflusst. Es ergab sich somit kein Hinweis auf eine mögliche fokale Gliose oder Neurodegeneration wie in der MRT-Arbeit von Schneider et al. postuliert ${ }^{92}$. In dieser Studie waren die im Putamen und Globus pallidus gemessenen T2-Zeiten in einer Gruppe von 17 Patienten mit zervikaler Dystonie gegenüber einer gesunden Kontrollkohorte signifikant verlängert. Dies wurde mit einer möglichen Vergrößerung des Extrazellularraumes durch 
Zellverlust mit nachfolgender Gliose erklärt. Die Daten dieser älteren Studie wurden allerdings mit einem 2-Tesla MRT-Scanner mit einer deutlich gröberen Auflösung (5 mm Schichtdicke, 7,5 m Schichtabstand) als in der vorliegenden Studie (1 $\mathrm{mm}$ isotrope Auflösung) erhoben. Insofern könnten Partialvolumeneffekte durch den benachbarten Liquorraum zu den erhöhten T2-Werten bei Dystoniepatienten beigetragen haben (Liquor hat eine deutlich höhere T2-Relaxationszeit als Hirngewebe). Da anzunehmen ist, dass sich Patienten mit einer Bewegungsstörung während der Messung durchschnittlich etwas mehr bewegen als Kontrollpersonen, könnte der Partialvolumeneffekt in der Studie von Schneider et al. als Folge der gröberen Auflösung auch stärker ausgeprägt gewesen sein. Die demographischen Daten der in beiden Studien untersuchten Kohorten sind hingegen relativ gleichartig (Schneider et al.: Patienten im Durchschnitt 2,6 Jahre jünger und 3,1 Jahre kürzer erkrankt) und somit nicht geeignet, die diskrepanten Ergebnisse zu erklären. Zusammenfassend ergaben sich in der vorliegenden Studie unter Berücksichtigung der methodischen Limitationen und der kleinen Fallzahl keine Hinweise auf Dystonie-assoziierte neurodegenerative Prozesse, die mit Veränderungen der T2-Zeit einhergehen, wie beispielsweise Gliose.

\subsubsection{T1-Relaxationszeit}

In dieser Studie wurden erstmalig Messungen der T1-Relaxationszeit bei Patienten mit idiopathischer Dystonie durchgeführt. Es konnten weder in der gesamten Kohorte noch in der Untergruppe der Patienten mit zervikaler Dystonie signifikante Veränderungen festgestellt werden. Lediglich eine Tendenz zu erhöhten T1-Zeiten konnte in den Bereichen der tiefen Grauen Substanz $(p=0,07)$, des Ncl. caudatus $(p=0,07)$, Putamen $(p=0,09)$ und Thalamus $(p=0,05)$ gefunden werden. Dieser Trend konnte in der Subgruppe der Patienten mit zervikaler Dystonie nicht bestätigt werden. Hier zeigten sich hingegen Tendenzen zu erhöhten T1-Zeiten in der Weißen Substanz $(p=0,05)$ und dem Thalamus $(p=0,09)$. Diese an der Grenze zur Signifikanz liegenden Ergebnisse sind schwierig zu interpretieren. Einerseits könnte es sich um rein zufällige Schwankungen handeln (es ist nicht anzunehmen, dass sich die Pathophysiologie zwischen Patienten mit 
unterschiedlichen Dystonieformen derartig grundlegend unterscheidet). Andererseits besteht die Möglichkeit, dass ein Fehler zweiter Art (beta-Fehler) vorliegt (d.h. die Nullhypothese wird fälschlicherweise angenommen, obwohl die Alternativhypothese korrekt ist ${ }^{113}$ ). Insgesamt kann also angesichts der kleinen Fallzahl nicht sicher ausgeschlossen werden, dass nicht doch geringe Abweichungen der T1-Zeit bei Patienten mit fokaler idiopathischer Dystonie bestehen.

Die hier gefundenen Ergebnisse der mittleren T1-Relaxationszeit stimmen dabei mit Werten von gesunden Probanden an 3-Tesla MRT-Scannern aus der Literatur überein 100, 114-116. Die angewandte Methode zur Bestimmung der T1-Relaxationszeiten ist bereits mehrfach in vorangegangenen Studien zur Untersuchung von mikrostrukturellen Veränderungen bei Patienten mit Multipler Sklerose genutzt worden69, 117, 118. Der Gruppenvergleich von T1-Werten ermöglicht Aussagen über mögliche Assoziationen zu Gewebeschäden wie Demyelinisierung, Gliose und axonalem Schaden. So weisen verlängerte T1-Zeiten bei Patienten mit Multipler Sklerose im Bereich der chronischen Läsionen auf einen fokalen Gewebeschaden einschließlich axonalem Verlust, axonaler Dysfunktion, erhöhtem Extrazellularvolumen sowie möglicherweise Gliose hin ${ }^{66,119}$. Zusammenfassend fanden sich in dem kleinen untersuchten Kollektiv keine signifikanten Veränderungen der T1-Relaxationszeit in den Basalganglien, der Weißen Substanz, dem Thalamus und Cerebellum, die potentiell hinweisend auf einen möglichen Gewebeschaden bei fokaler idiopathischer Dystonie sein könnten.

\subsubsection{Protonendichte}

Auch die Messung der PD ergab keine Abweichungen zwischen der Patientenund Kontrollkohorte. Diese wurde bisher nicht bei idiopathischen Dystonien untersucht. Die PD spiegelt die Menge der Protonen im untersuchten Gewebe wieder und wird daher zur Quantifizierung des Wassergehaltes im Gehirn genutzt ${ }^{64}$. Verschiedene neurologische Erkrankungen führen zu einer Erhöhung der PD. Dazu zählen beispielsweise Hirntumore ${ }^{120}$, der ischämische Schlaganfall ${ }^{121}$ und die hepatische Enzephalopathie ${ }^{65}$. Die Erhöhung des Wassergehaltes entsteht 
dabei häufig aufgrund von Inflammation oder Ödembildung. Das negative Ergebnis in dieser Studie legt nahe, dass Neuroinflammation und Ödembildung bei fokalen idiopathischen Dystonien keine wesentlichen Pathomechanismen darstellen. Dabei waren auch hier die PD-Werte vergleichbar mit Werten aus vorangegangenen Studien an gesunden Probanden ${ }^{105,115,116}$. Einzig auffällig sind die erhöhten Standardabweichungen und Spannweiten der Werte v.a. im Bereich der Basalganglien und der Weißen Substanz in der Dystonie-Kohorte. Dies lässt sich bei nicht signifikantem Unterschied der Mittelwerte zwischen den Gruppen am ehesten durch Bewegungsartefakte erklären. Insgesamt fanden sich in dieser Studie keine signifikanten Veränderungen der PD bei fokaler idiopathischer Dystonie, die auf Prozesse einer möglichen Inflammation oder Ödembildung hinweisen könnten.

\subsection{Verknüpfung bildgebender Studien mit der Histopathologie}

Die Untersuchung der Basalganglien, des Thalamus, der Weißen Substanz und des Cerebellums mittels qMRT ergab somit keine Hinweise auf mikrostrukturelle Veränderungen weder im heterogenen Gesamtkollektiv noch in der Subgruppe der Patienten mit zervikaler Dystonie. Dies deckt sich in groben Zügen mit den Ergebnissen der wenigen histopathologischen Untersuchungen zu dieser Erkrankungsgruppe. Bislang konnten keine übereinstimmenden Pathologien bei der expliziten Untersuchung der Basalganglien mit verschiedenen Färbemethoden festgestellt werden ${ }^{47-49,122}$. Jedoch konnten Prudente et al. einen Verlust von Purkinje-Zellen, Areale fokaler Gliose und Sphäroide (geschwollene Axonzylinder der Purkinje-Zellen) im Cerebellum nachweisen ${ }^{48}$. Weiterhin zeigten einzelne Fallserien Nervenzellverluste in Kerngebieten des Hirnstamms ${ }^{47}$ und der Substantia nigra ${ }^{6}$. Die Hirnstammkerngebiete sind in der hiesigen Studie nicht untersucht worden, da diese aufgrund ihrer kleinen Größe nicht in den Segmentierungsalgorithmen von Freesurfer enthalten sind. Auch lassen sie sich aufgrund des geringen Volumens nicht sinnvoll mit einem voxelbasierten statistischen Ansatz in einem Standardraum analysieren. Änderungen von qMRT-Parametern in 
den Hirnstammkerngebieten können anhand dieser Studie also weder positiv belegt noch ausgeschlossen werden.

Es sollte ebenfalls beachtet werden, dass in den oben genannten histopathologischen Studien verschiedene methodische Limitationen vorliegen. Mit Ausnahme der Studie von Prudente et al. ${ }^{48}$ wurden alle Untersuchungen ohne Gruppenvergleich zu einer Kontrollkohorte durchgeführt. Weiterhin wurden nur in modernen Arbeiten immunhistologische Färbungen angewendet. Ferner wurden lediglich einzelne vorausgewählte Hirnregionen, häufig mit dem Fokus auf den Basalganglien, untersucht. Letztlich konnte bislang einzig der Verlust von PurkinjeZellen bei Patienten mit zervikaler Dystonie quantitativ bestätigt werden ${ }^{48}$. Histopathologische Untersuchungen gelten dennoch weiterhin als Goldstandard zum Aufzeigen von Pathologien, da Gewebeveränderungen direkt sichtbar gemacht werden können.

Die ursprüngliche Hypothese einer alleinigen Pathologie in den Basalganglien von fokalen idiopathischen Dystonien wird somit nicht durch histopathologische Untersuchungen unterstützt. Diese Sichtweise hat ihren Ursprung darin, dass bei sekundären Dystonien wiederholt Läsionen im Putamen, Ncl. Caudatus und Thalamus gefunden wurden ${ }^{45}$, 46. Weiterhin bekräftigt das funktionelle Modell der Basalganglien diesen Ansatz (siehe Kapitel 1.1.2 Pathophysiologie). Jedoch werden erworbene Dystonien tatsächlich am häufigsten durch Läsionen im Cerebellum oder dem Hirnstamm ausgelöst ${ }^{123}$. Seltener ist eine offenbare strukturelle Ursache in den Basalganglien oder dem Rückenmark zu finden. Moderne bildgebende Studien, die beispielsweise die Methodik der fMRT nutzten, zeigten ebenfalls eine alleinige oder kombinierte Beteiligung des zerebralen Kortex, der Basalganglien und/oder des Cerebellums ${ }^{43}$. Auch mittels Fluorodeoxyglucose-Positronen-Emissions-Tomographie, bei der mittels radioaktiv markierter Biomoleküle Veränderungen im Zellstoffwechsel sichtbar gemacht werden können, konnte eine Beteiligung verschiedener motorischer Areale (u.a. zerebraler Kortex, Cerebellum, Basalganglien, Hirnstamm) aufgezeigt werden ${ }^{43}$. Somit stellen auch funktionelle bildgebende Studien das pathophysiologische Modell einer reinen Basalganglien-Pathologie in Frage. Dieser Standpunkt wird ebenso dadurch 
unterstützt, dass nicht jeder Patient mit Basalganglienläsionen dystone Symptome zeig ${ }^{45}$. Ferner weisen Studien an Tiermodellen neben der Beteiligung der Basalganglien auf eine Dysfunktion des Cerebellums und des Hirnstammes $\mathrm{hin}^{124}$. Diese lockere Korrelation von Basalganglienbeteiligung und Dystonie bekräftigt die Sichtweise, dass wahrscheinlich multiple Hirnregionen in der Entstehung von Dystonien involviert sind.

Diese Hypothese wird auch durch mehrere DTI-Studien unterstützt, welche Veränderungen der Faserverbindungen zwischen den Basalganglien, dem zerebralen Kortex und dem Cerebellum nachweisen konnten ${ }^{77}$. Colosimo et al. konnten bei Patienten mit zervikaler Dystonie eine Erhöhung der FA im Putamen und eine Erniedrigung der MD im linken Pallidum und Putamen sowie bilateral im Ncl. caudatus nachweisen ${ }^{90}$. Weitere Untersuchungen zeigten eine erhöhte FA im Thalamus und der benachbarten Weißen Substanz ${ }^{125}$ sowie eine erhöhte MD in der umgebenden Weißen Substanz von Pallidum und Putamen beidseits, im linken $\mathrm{Ncl}$. caudatus und subkortikal des Gyrus postzentralis ${ }^{125,}{ }^{126}$. Es liegen folglich Hinweise auf mikrostrukturelle Veränderungen in verschiedenen Regionen motorischer Kontrolle bei idiopathischen Dystonien vor, jedoch sind die Ergebnisse bisheriger DTI-Studien heterogen und teilweise inkonsistent. Weiterhin kann nicht ausgeschlossen werden, dass Änderungen z.B. der Faserdichte nicht die Ursache der Erkrankung, sondern Folge einer anderen Pathologie darstellen.

\subsection{Volumenveränderungen bei idiopathischen Dystonien}

Vorausgegangene konventionelle MRT-Studien erbrachten bislang widersprüchliche Erkenntnisse zu Volumenveränderungen in den Basalganglien und anderen Hirnregionen. So wurde beispielsweise ein erhöhtes Volumen der Grauen Substanz im Putamen nachgewiesen ${ }^{78,79}$, andere Untersuchungen hingegen beschrieben ein erniedrigtes Volumen für dieses Kerngebiet ${ }^{80-82}$. Weitere Studien wiederum zeigten keine Volumenänderungen im Putamen auf, stattdessen jedoch in anderen Basalganglienkernen, v.a. im Globus pallidus internus ${ }^{83,84}$. Andere Veröffentlichungen dagegen fanden keine signifikanten Änderungen der 
Volumina der Basalganglienkerne, stattdessen jedoch erniedrigte und/oder erhöhte Volumina im Thalamus ${ }^{80,85}$, Cerebellum ${ }^{80,85,127}$ oder Bereichen des zerebralen Kortex ${ }^{83,86,127}$, insbesondere der sensomotorischen und prämotorischen Regionen. In dieser Studie konnte mittels FreeSurfer keine Abweichung des Volumens im Pallidum, Putamen, Thalamus, der Weißen Substanz, dem zerebralen Kortex und Cerebellum bei Patienten mit fokaler idiopathischer Dystonie nachgewiesen werden. Eine voxelbasierte Untersuchung einzelner kortikaler Areale wurde allerdings in dieser Arbeit nicht vorgenommen. Zusammenfassend ergaben sich in dem untersuchten Kollektiv keine Hinweise auf eine mit der Dystonie assoziierte Atrophie (oder Hemiatrophie) im Großhirnkortex, der Weißen Substanz, dem Cerebellum, den Basalganglien oder dem Thalamus. Somit konnte auch das von Schneider et al. beschriebene leicht gehäufte Vorkommen einer Hemiatrophie bei Patienten mit zervikaler Dystonie ${ }^{92}$ nicht bestätigt werden. Diese wurde von den Autoren bei nicht signifikantem Unterschied zwischen den Gruppen als unspezifisch interpretiert. Die negativen Ergebnisse auch der Volumetrie unterstützen die Hypothese, dass Neurodegeneration und Atrophie selbst nach mehrjähriger Erkrankungsdauer keine größere Rolle in der Pathophysiologie von fokalen idiopathischen Dystonien zu spielen scheinen.

\subsection{Korrelation des klinischen Schweregrades mit bildgebenden Parametern}

Die fehlende Korrelation zwischen den Gewebeparametern der tiefen Grauen Substanz und dem Tsui-Score zum Zeitpunkt der MRT ist aufgrund der nicht signifikanten Gruppenunterschiede der qMRT-Parameter wenig überraschend. Der Tsui-Score wird durch die Botulinumtoxin-Wirkung beeinflusst und bildet daher nur begrenzt den „wahren" Schweregrad der Erkrankung ab. Er wird überwiegend in der Therapiekontrolle als Messinstrument eingesetzt. Dabei gelten der Tsui-Score und die TWSTRS beide als valide Methoden zur Erfassung der Symptomverbesserung bei zervikaler Dystonie ${ }^{128}$. Auch Aschermann et al. konnten in ihrer Studie keine Korrelation zwischen T2*-Zeiten und der TWSTRS nachwei$\operatorname{sen}^{91}$. Piccinin et al. hingegen konnten eine positive Korrelation zwischen dem 
Volumen u.a. des rechten präzentralen Gyrus und der Fahn-Marsden Dystonia Rating Scale (FMDRS) bei Patienten mit kraniozervikaler Dystonie messen ( $\mathrm{n}=$ $27)^{127}$. Diese Skala dient zur Beurteilung des Schweregrades sowie der Einschränkung durch eine Dystonie und wird überwiegend zur Evaluation von generalisierten Dystonien eingesetzt ${ }^{129}$. Sie ist dementsprechend nicht optimal zur Beurteilung von kraniozervikalen Dystonien geeignet, es sollte eher auf die TWSTRS oder den Craniocervical Dystonia Questionnaire (CDQ-24) zurückgegriffen werden. Zusammenfassend konnte bislang in den wenigen durchgeführten Untersuchungen keine eindeutige Korrelation zwischen einer klinischen Beurteilungsskala für fokale Dystonien und MRT-Parametern gefunden werden.

\subsection{Limitationen}

Die relativ kleine Fallzahl der Kohorten mit $\mathrm{n}=26$ entspricht in etwa den Fallzahlen der vorangegangenen qMRT-Studien $(n=12-17)^{91,92}$. Eine wesentlich höhere Probandenzahl ist bei einer monozentrisch durchgeführten Studie angesichts des seltenen Vorkommens der Erkrankung in einem überschaubaren Zeitraum nur schwer zu erlangen. Die klinischen Charakteristika der Patientenkohorte (Frauenanteil, Verteilung und Manifestationsalter der Dystonieformen uvm.) stimmen ungefähr mit den epidemiologischen Daten der Literatur überein $7,21,22$. Die kollektive Auswertung heterogener Unterformen fokaler Dystonien zum Aufzeigen einer gemeinsamen Pathologie ist nicht ungewöhnlich ${ }^{79,80,82}$. Jedoch liegt eine Limitation in der Überrepräsentation von Patienten mit zervikaler Dystonie $(n=17)$ im Vergleich zu den anderen Dystonieformen $(n=9)$ vor. Somit ist eine Verzerrung der Ergebnisse nicht auszuschließen. Weiterhin ist aufgrund des heterogenen Gesamtkollektives von einer erhöhten Variabilität der untersuchten Effektgröße auszugehen, was die Sensitivität der statistischen Tests beeinflusst und die Wahrscheinlichkeit für einen Fehler zweiter Art erhöht. Die Wahrscheinlichkeit eines solchen Fehlers ist bei einer kleinen Stichprobe (wie hier) und den ohnehin zu erwartenden kleinen Effektgrößen besonders hoch. Dies ist insbesondere aufgrund des negativen Studienergebnisses relevant. Um diesen Kritikpunkt zu umgehen, wurde zusätzlich eine Subgruppenanalyse, in der lediglich 
Patienten mit zervikaler Dystonie berücksichtigt wurden, durchgeführt. Aufgrund der ebenfalls nicht signifikanten Resultate scheinen zumindest grobe Veränderungen in den untersuchten qMRT-Gewebeparametern bei idiopathischen Dystonien tatsächlich nicht vorzuliegen.

Auch sollte eine weitere generelle Limitation der qMRT-Methodik bedacht werden. So lag beispielsweise die scan-rescan Variabilität von T1-Messungen in einer Untersuchung bei rund 3\% ${ }^{106}$, bei T2/T2*-Messungen können sogar noch höhere Variabilitäten angenommen werden ${ }^{130,131}$. Kleine Änderungen der Effektgröße, die unterhalb dieser Messgenaugkeitsgrenze liegen, sind nur mit größeren Kohorten sicher detektierbar und könnten daher in dieser Studie übersehen worden sein (Fehler zweiter Art). Positiv an dieser Arbeit ist zu bewerten, dass verschiedene quantitative Gewebeparameter gleichzeitig erhoben und diese mittels zweier unterschiedlicher statistischer Auswerteverfahren analysiert worden sind.

\subsection{Idiopathische Dystonien als neurofunktionelle Störung}

Zusammenfassend konnte mittels multimodaler qMRT im untersuchten Kollektiv keine den fokalen idiopathischen Dystonien zugrunde liegenden mikrostrukturellen Veränderungen nachgewiesen werden. Bereits seit mehreren Jahren wird aufgrund zahlreicher moderner bildgebender Studien und Versuchen an Tiermodellen, die auf eine Beteiligung mehrerer Hirnregionen in der Pathophysiologie von idiopathischen Dystonien hinweisen, diskutiert, ob diese primär durch eine neurofunktionelle Störung hervorgerufen werden $43,124,132,133$. Unterstützt wird diese Hypothese u.a. durch das Fehlen von makroskopischen Gewebeveränderungen und unschlüssigen mikroskopischen Pathologien. Eine Netzwerkstörung kann sich durch Veränderungen der neuronalen Konnektivität, Plastizität und/oder der synaptischer Regulation entwickeln ${ }^{9}$. Das „motor network moder ${ }^{443}$ postuliert, dass alle Subtypen der Dystonie nicht durch eine Dysfunktion einzelner Hirnregionen, sondern durch eine Dysfunktion oder abnormale Kommunikation innerhalb der sensomotorischen Netzwerke entstehen. Zu diesen 
Netzwerken zählen die Basalganglien, das Cerebellum, der Thalamus und der zerebrale Kortex. Das Model inkludiert dabei sehr gut die Ergebnisse diverser neurophysiologischer und funktioneller bildgebender Studien, die auf eine Beteiligung multipler Hirnregionen in der Entstehung dystoner Bewegungen hinwei$\operatorname{sen}^{43}$. Battistella et al. konnten beispielsweise eine großräumige Reorganisation von funktionellen Netzwerken bei Patienten mit isolierter fokaler Dystonie aufzeigen ${ }^{132}$. U.a. demonstrierten sie eine Aufspaltung des großen funktionellen Netzwerkes bestehend aus den Basalganglien, dem Thalamus und Cerebellum in multiple kleinere Netzwerke. So reduzierten sich die beteiligten Kerngebiete dieses großen Netzwerkes signifikant, das sich anschließend nur noch auf einige Regionen des Cerebellums beschränkte. Gleichzeitig konnte eine funktionelle Reorganisation der Basalganglien und des Thalamus nachgewiesen werden. Eine andere Untersuchung an Patienten mit Schreibkrampf zeigte Veränderungen der funktionellen Konnektivität in der cerebello-basalganglien-thalamo-kortikalen Schleife ${ }^{134}$. Eine weitere Studie konnte Unterschiede in der Hirnaktivität in den sensomotorischen Netzwerken (u.a. zwischen dem Ncl. caudatus und dem primären sensomotorischen Kortex, dem Cerebellum und dem somatosensorischen sowie visuellen Kortex, prämotorischer Areale und dem primären somatosensorischen Kortex uvm.) bei Patienten mit Blepharospasmus nachweisen ${ }^{135}$. Diese funktionellen Veränderungen zeigten sich bei erfolgreicher symptomatischer Therapie mit Botulinumtoxin ${ }^{135,136}$ und einem experimentellen Ansatz mit Natriumoxybat ${ }^{137}$ rückläufig. Die Ergebnisse der genannten Studien legen eine neurofunktionelle Störung als kausale Ursache idiopathischer Dystonien nahe. Auch die Ergebnisse der hier vorgestellten Untersuchung unterstützen indirekt diesen Ansatz, da das Fehlen offenbarer mikrostruktureller Veränderungen eine funktionelle Störung als Ursache der Bewegungsstörung notwendig erscheinen lässt. Die Tatsache, dass strukturelle Defekte in den Basalganglien gewöhnlich erst nach Wochen oder Jahren zu klinischen Manifestationen einer Dystonie führen $^{138,139}$, weist auf eine sich im Rahmen einer Maladaption langsam entwickelnden Netzwerkdysfunktion hin. Dies impliziert, dass nicht unbedingt der Funktionsverlust der Läsionen selbst die Ursache erworbener Dystonien ist. 
Unbeantwortet bleibt, ob diese denkbaren neurofunktionellen Störungen primär, sekundär oder kompensatorisch entstehen und welche Rolle einzelne Regionen der motorischen Kontrolle in diesem Netzwerk spielen. Die Beantwortung der Frage, ob diesen neurofunktionellen Veränderungen nicht doch minimale mikrostrukturelle Läsionen zugrunde liegen, die unterhalb des Auflösungsvermögens und der Genauigkeitsgrenze der hier angewandten Methoden liegen, bleibt naturgemäß offen. MRT-Studien an größeren Kollektiven in Kombination mit quantitativen histologischen Untersuchungen sind erforderlich, um die pathophysiologischen Grundlagen von fokalen idiopathischen Dystonien weiter zu erhellen. 


\section{Zusammenfassung}

Fokale idiopathische Dystonien stellen die häufigste Dystonieform im Erwachsenenalter dar. Die Pathophysiologie dieser Erkrankungsgruppe ist weitestgehend unverstanden, wobei die Basalganglien, der Thalamus und das Cerebellum eine zentrale Rolle in der Genese dystoner Bewegungen zu spielen scheinen. Unklar ist, ob Patienten mit fokaler idiopathischer Dystonie mikrostrukturelle Veränderungen in den oben genannten Arealen aufweisen, die das Störungsbild bedingen könnten.

In dieser Arbeit wurde mittels Methoden der quantitativen Magnetresonanztomographie (qMRT) der Versuch unternommen, Änderungen von Struktur und Eigenschaften des Hirngewebes bei idiopathischen Dystonien im Vergleich zu einer gesunden Kontrollkohorte zu identifizieren. Vorangegangen bildgebende Studien erbrachten bislang widersprüchliche Ergebnisse. Insbesondere der Frage nach möglichen Veränderungen des Eisengehaltes sollte mittels Messung der T2*-Relaxationszeit nachgegangen werden. Weiterhin wurden Areale der motorischen Kontrolle (Basalganglien, Thalamus, Cerebellum und zerebraler Kortex) auf mögliche Volumenveränderungen untersucht.

Insgesamt wurden 30 Patienten mit fokaler idiopathischer Dystonie sowie 30 alters- und geschlechtsgematchte Kontrollprobanden mittels multimodaler qMRT untersucht und Parameterkarten für die T1- und T2/T2*-Relaxationszeiten sowie der Protonendichte berechnet. Die Parameterkarten wurden sowohl voxelweise als auch regionenbasiert mit der Frage nach Dystonie-spezifischen Veränderungen statistisch ausgewertet. Zusätzlich erfolgte eine Subgruppenanalyse der genannten Parameter von 17 Patienten mit zervikaler Dystonie im Vergleich zu einer verkleinerten Kontrollgruppe.

Für keinen der untersuchten qMRT-Parameter konnte in der voxelweisen oder der regionenbasierten Analyse signifikante Gruppenunterschiede zwischen Patienten mit fokaler idiopathischer Dystonie und gesunden Kontrollprobanden nachgewiesen werden $(p \geq 0,05)$. Auch unterschieden sich die untersuchten 
Hirnregionen nicht hinsichtlich ihres Volumens $(p \geq 0,31)$. Ebenfalls ausschließlich negative Ergebnisse ergab die Subgruppenanalyse für Patienten mit zervikaler Dystonie (Gewebeparameter $p \geq 0,05$, Volumen $p \geq 0,21$ ).

Somit fanden sich entgegen der ursprünglichen Hypothese keine mittels qMRT detektierbaren krankheitsspezifischen mikrostrukturellen Gewebeveränderungen bei Patienten mit fokaler idiopathischer Dystonie. Unter Berücksichtigung der methodischen Limitationen und der kleinen Fallzahl ergaben sich keine Hinweise auf Dystonie-assoziierte neurodegenerative Prozesse, erhöhte Eisenablagerungen, Demyelinisierung oder Veränderungen des Wassergehaltes. Die Ergebnisse dieser Studie sind kompatibel mit der Sichtweise, dass idiopathische Dystonien am ehesten aufgrund einer reinen neurofunktionellen Netzwerkstörung der Basalganglien und deren kortikalen sowie cerebellären Projektionsareale entstehen. Hierbei ist zu berücksichtigen, dass sehr kleine, feingewebliche Veränderungen, die unterhalb des Auflösungsvermögens der hier verwendeten Bildgebungsmethode liegen, nicht sicher ausgeschlossen werden können. Weitere quantitativ histologische Untersuchungen in Kombination mit quantitativ bildgebenden Verfahren werden benötigt, um die Pathophysiologie dieser Erkrankungsgruppe besser verstehen zu können. 


\section{Abstract}

Idiopathic focal dystonia is the most common type of dystonia in adult life. Yet, the pathophysiology of this disease is not well understood. Important structures in disease cause are the basal ganglia, cortico-striathothalamic circuits and the cerebellum. It is unclear whether patients with idiopathic focal dystonia have microstructural changes in the areas mentioned above that could cause the disorder.

Therefore, the present study aimed to identify potential microstructural cerebral changes in patients with idiopathic focal dystonia compared to a healthy control cohort using multimodal quantitative magnetic resonance imaging (qMRI). Previous qMRI studies which have examined focal dystonia yielded conflicting results. In particular, the question of possible changes in the iron content was investigated by measuring the $T 2^{*}$-relaxation time. In addition, areas of motor control (basal ganglia, thalamus, cerebellum and cerebral cortex) were examined for volume modifications.

Mapping of T1-, T2/T2*-relaxation times and proton density was performed in 30 patients with idiopathic focal dystonia and 30 healthy control subjects matched for age and gender. Between group comparisons were accomplished for each qMRI parameter using statistical parametric mapping. Additionally, regions of interest (ROI) based analyses were carried out for the major basal ganglia nuclei, thalamus, white matter and cerebellar grey and white matter. Furthermore, a subgroup comparison was performed for patients with cervical dystonia $(n=17)$.

Neither the voxel-based nor the ROI-based analysis revealed significant group differences for any qMRI parameter under investigation $(p \geq 0,05)$. Likewise, $R O I$ volumes did not differ between the groups $(p \geq 0,31)$. A repetition of the analysis for the subgroup of patients with cervical dystonia also yielded only negative findings (tissue parameter $p \geq 0,05$, volume $p \geq 0,21$ ).

Thus, in contrast to the original hypothesis no disease-specific microstructural tissue changes were detected by qMRI in patients with idiopathic focal dystonia. 
Considering the methodological limitations and the small number of cases, the study revealed no evidence for dystonia-associated neurodegenerative processes, such as increased iron deposition, demyelination or changes in water content. These negative results are compatible with the view that idiopathic dystonia might be a pure neurofunctional network disorder characterized by a disturbed neuronal communication between the basal ganglia and their cortical and cerebellas projections areas. However, it cannot be ruled out that minor microstructural tissue changes below the resolution of qMRI also contribute to the disease. Further quantitative histological studies in combination with quantitative imaging techniques are required to untangle the pathophysiology of idiopathic dystonia. 


\section{Literaturverzeichnis}

1. Albanese A, Asmus F, Bhatia KP, et al. EFNS guidelines on diagnosis and treatment of primary dystonias. Eur J Neurol. 2011;18(1):5-18. doi:10.1111/j.1468-1331.2010.03042.x.

2. Ceballos-Baumann AO. Dystonien. In: Ceballos-Baumann AO, Conrad B, eds. Bewegungsstörungen. s.l.: THIEME; 2005:128-180.

3. Lauterbach EC, Freeman A, Vogel RL. Differential DSM-III psychiatric disorder prevalence profiles in dystonia and Parkinson's disease. J Neuropsychiatry Clin Neurosci. 2004;16(1):29-36. doi:10.1176/jnp.16.1.29.

4. Gündel H, Wolf A, Xidara V, Busch R, Ceballos-Baumann AO. Social phobia in spasmodic torticollis. J Neurol Neurosurg Psychiatry. 2001;71(4):499504. doi:10.1136/jnnp.71.4.499.

5. Nutt JG, Muenter MD, Aronson A, Kurland LT, Melton LJ. Epidemiology of focal and generalized dystonia in Rochester, Minnesota. Mov Disord. 1988;3(3):188-194. doi:10.1002/mds.870030302.

6. lacono D, Geraci-Erck M, Peng H, Rabin ML, Kurlan R. Reduced Number of Pigmented Neurons in the Substantia Nigra of Dystonia Patients? Findings from Extensive Neuropathologic, Immunohistochemistry, and Quantitative Analyses. Tremor Other Hyperkinet Mov (N Y). 2015;5. doi:10.7916/D8T72G9G.

7. Sex-related influences on the frequency and age of onset of primary dystonia. Epidemiologic Study of Dystonia in Europe (ESDE) Collaborative Group. Neurology. 1999;53(8):1871-1873.

8. Oppenheim H. Über eine eigenartige Krampfkrankheit des kindlichen und jugendlichen Alters (Dysbasia lordotica progressiva, Dystonia musculorum deformans). Neurol. Cbl. 1911; 30:1090-1107. 
9. Breakefield XO, Blood AJ, Li Y, Hallett M, Hanson PI, Standaert DG. The pathophysiological basis of dystonias. Nat Rev Neurosci. 2008;9(3):222234. doi:10.1038/nrn2337.

10. Albanese A, Bhatia K, Bressman SB, et al. Phenomenology and classification of dystonia. A consensus update. Mov Disord. 2013;28(7):863-873. doi:10.1002/mds.25475.

11. Balint B, Bhatia KP. Dystonia. An update on phenomenology, classification, pathogenesis and treatment. Curr Opin Neurol. 2014;27(4):468-476. doi:10.1097/WCO.0000000000000114.

12. Tinazzi M, Rosso T, Fiaschi A. Role of the somatosensory system in primary dystonia. Mov Disord. 2003;18(6):605-622. doi:10.1002/mds.10398.

13. Diener H-C. Leitlinien für Diagnostik und Therapie in der Neurologie: Herausgegeben von der Kommission quot;Leitlinienquot; der DGN. 5th ed. s.I.: Georg Thieme Verlag KG; 2012.

14. Batla A. Dystonia. A review. Neurol India. 2018;66(Supplement):S48-S58. doi:10.4103/0028-3886.226439.

15. Albanese A, Di Giovanni M, Lalli S. Dystonia. Diagnosis and management. Eur J Neurol. 2018. doi:10.1111/ene.13762.

16. Rosencrantz R, Schilsky M. Wilson disease. Pathogenesis and clinical considerations in diagnosis and treatment. Semin Liver Dis. 2011;31(3):245259. doi:10.1055/s-0031-1286056.

17. Klein C, Ozelius LJ. Dystonia. Clinical features, genetics, and treatment. Curr Opin Neurol. 2002;15(4):491-497.

18. Ozelius LJ, Hewett JW, Page CE, et al. The early-onset torsion dystonia gene (DYT1) encodes an ATP-binding protein. Nat Genet. 1997;17(1):4048. doi:10.1038/ng0997-40. 
19. Fahn S. Classification of movement disorders. Mov Disord. 2011;26(6):947957. doi:10.1002/mds.23759.

20. Defazio G, Abbruzzese G, Livrea P, Berardelli A. Epidemiology of primary dystonia. Lancet Neurol. 2004;3(11):673-678. doi:10.1016/S14744422(04)00907-X.

21. Castelon Konkiewitz E, Trender-Gerhard I, Kamm C, et al. Service-based survey of dystonia in munich. Neuroepidemiology. 2002;21(4):202-206. doi:10.1159/000059525.

22. Asgeirsson $\mathrm{H}$, Jakobsson $\mathrm{F}$, Hjaltason $\mathrm{H}$, Jonsdottir $\mathrm{H}$, Sveinbjornsdottir $\mathrm{S}$. Prevalence study of primary dystonia in Iceland. Mov Disord. 2006;21(3):293-298. doi:10.1002/mds.20674.

23. Chan J, Brin MF, Fahn S. Idiopathic cervical dystonia. Clinical characteristics. Mov Disord. 1991;6(2):119-126. doi:10.1002/mds.870060206.

24. Jankovic J, Leder S, Warner D, Schwartz K. Cervical dystonia. Clinical findings and associated movement disorders. Neurology. 1991;41(7):10881091.

25. Mordin M, Masaquel C, Abbott C, Copley-Merriman C. Factors affecting the health-related quality of life of patients with cervical dystonia and impact of treatment with abobotulinumtoxinA (Dysport). Results from a randomised, double-blind, placebo-controlled study. BMJ Open. 2014;4(10):e005150. doi:10.1136/bmjopen-2014-005150.

26. Geyer HL, Bressman SB. The diagnosis of dystonia. Lancet Neurol. 2006;5(9):780-790. doi:10.1016/S1474-4422(06)70547-6.

27. Kutvonen O, Dastidar P, Nurmikko T. Pain in spasmodic torticollis. Pain. 1997;69(3):279-286. 
28. Velickovic M, Benabou R, Brin MF. Cervical dystonia pathophysiology and treatment options. Drugs. 2001;61(13):1921-1943. doi:10.2165/00003495200161130-00004.

29. Jahanshahi M. Factors that ameliorate or aggravate spasmodic torticollis. $J$ Neurol Neurosurg Psychiatry. 2000;68(2):227-229.

30. Dauer WT, Burke RE, Greene P, Fahn S. Current concepts on the clinical features, aetiology and management of idiopathic cervical dystonia. Brain. 1998;121 ( Pt 4):547-560.

31. Defazio G, Livrea P. Primary blepharospasm. Diagnosis and management. Drugs. 2004;64(3):237-244. doi:10.2165/00003495-200464030-00002.

32. Colosimo C, Suppa A, Fabbrini G, Bologna M, Berardelli A. Craniocervical dystonia: clinical and pathophysiological features. Eur J Neurol. 2010;17 Suppl 1:15-21. doi:10.1111/j.1468-1331.2010.03045.x.

33. Hallett M, Evinger C, Jankovic J, Stacy M. Update on blepharospasm. Report from the BEBRF International Workshop. Neurology. 2008;71(16):1275-1282. doi:10.1212/01.wnl.0000327601.46315.85.

34. Lee JM, Baek JS, Choi HS, Kim SJ, Jang JW. Clinical Features of Benign Essential Blepharospasm in Korean Patients. Korean J Ophthalmol. 2018;32(5):339-343. doi:10.3341/kjo.2018.0038.

35. Hall TA, McGwin G, Searcey K, et al. Benign essential blepharospasm. Risk factors with reference to hemifacial spasm. $J$ Neuroophthalmol. 2005;25(4):280-285.

36. Defazio G, Livrea P. Epidemiology of primary blepharospasm. Mov Disord. 2002;17(1):7-12.

37. Cossu G, Mereu A, Deriu M, et al. Prevalence of primary blepharospasm in Sardinia, Italy. A service-based survey. Mov Disord. 2006;21(11):20052008. doi:10.1002/mds.21084. 
38. Greene P, Kang UJ, Fahn S. Spread of symptoms in idiopathic torsion dystonia. Mov Disord. 1995;10(2):143-152. doi:10.1002/mds.870100204.

39. Lee KH. Oromandibular dystonia. Oral Surg Oral Med Oral Pathol Oral Radiol Endod. 2007;104(4):491-496. doi:10.1016/j.tripleo.2007.04.001.

40. Goldman JG. Writer's cramp. Toxicon. 2015;107(Pt A):98-104. doi:10.1016/j.toxicon.2015.09.024.

41. Hallett M. Pathophysiology of writer's cramp. Hum Mov Sci. 2006;25(45):454-463. doi:10.1016/j.humov.2006.05.004.

42. A prevalence study of primary dystonia in eight European countries. J Neurol. 2000;247(10):787-792.

43. Neychev VK, Gross RE, Lehéricy S, Hess EJ, Jinnah HA. The functional neuroanatomy of dystonia. Neurobiol Dis. 2011;42(2):185-201. doi:10.1016/j.nbd.2011.01.026.

44. Shakkottai VG, Batla A, Bhatia K, et al. Current Opinions and Areas of Consensus on the Role of the Cerebellum in Dystonia. Cerebellum. 2017;16(2):577-594. doi:10.1007/s12311-016-0825-6.

45. Bhatia KP, Marsden CD. The behavioural and motor consequences of focal lesions of the basal ganglia in man. Brain. 1994;117 ( Pt 4):859-876.

46. Marsden CD, Obeso JA, Zarranz JJ, Lang AE. The anatomical basis of symptomatic hemidystonia. Brain. 1985;108 ( Pt 2):463-483.

47. Zweig RM, Hedreen JC, Jankel WR, Casanova MF, Whitehouse PJ, Price DL. Pathology in brainstem regions of individuals with primary dystonia. Neurology. 1988;38(5):702-706.

48. Prudente CN, Pardo CA, Xiao J, et al. Neuropathology of cervical dystonia. Exp Neurol. 2013;241:95-104. doi:10.1016/j.expneurol.2012.11.019. 
49. Gibb WR, Lees AJ, Marsden CD. Pathological report of four patients presenting with cranial dystonias. Mov Disord. 1988;3(3):211-221. doi:10.1002/mds.870030305.

50. DeLong MR. Primate models of movement disorders of basal ganglia origin. Trends Neurosci. 1990;13(7):281-285.

51. Delong MR, Wichmann T. Circuits and circuit disorders of the basal ganglia. Arch Neurol. 2007;64(1):20-24. doi:10.1001/archneur.64.1.20.

52. Mink JW. THE BASAL GANGLIA: FOCUSED SELECTION AND INHIBITION OF COMPETING MOTOR PROGRAMS. Progress in Neurobiology. 1996;50(4):381-425. doi:10.1016/S0301-0082(96)00042-1.

53. Hallett M. Neurophysiology of dystonia: The role of inhibition. Neurobiol Dis. 2011;42(2):177-184. doi:10.1016/j.nbd.2010.08.025.

54. Marsden CD, Rothwell JC. The physiology of idiopathic dystonia. Can J Neurol Sci. 1987;14(3 Suppl):521-527.

55. Blood AJ, Flaherty AW, Choi J-K, et al. Basal ganglia activity remains elevated after movement in focal hand dystonia. Ann Neurol. 2004;55(5):744748. doi:10.1002/ana.20108.

56. Hu X-y, Wang L, Liu H, Zhang S-z. Functional magnetic resonance imaging study of writer's cramp. Chin Med J. 2006;119(15):1263-1271.

57. Preibisch C, Berg D, Hofmann E, Solymosi L, Naumann M. Cerebral activation patterns in patients with writer's cramp. A functional magnetic resonance imaging study. J Neurol. 2001;248(1):10-17.

58. Tofts $P$, Dowell NG, Cercignani M, eds. Quantitative MRI of the brain: Principles of physical measurement. Boca Raton, London, New York: CRC Press Taylor \& Francis Group; 2018. 
59. Venkatesan R, Lin W, Haacke EM. Accurate determination of spin-density and T1 in the presence of RF-field inhomogeneities and flip-angle miscalibration. Magn Reson Med. 1998;40(4):592-602.

60. Volz S, Nöth U, Rotarska-Jagiela A, Deichmann R. A fast B1-mapping method for the correction and normalization of magnetization transfer ratio maps at 3 T. Neuroimage. 2010;49(4):3015-3026. doi:10.1016/j.neuroimage.2009.11.054.

61. Reitz SC, Hof S-M, Fleischer V, et al. Multi-parametric quantitative MRI of normal appearing white matter in multiple sclerosis, and the effect of disease activity on T2. Brain Imaging Behav. 2017;11(3):744-753. doi:10.1007/s11682-016-9550-5.

62. Deoni SCL. Quantitative relaxometry of the brain. Top Magn Reson Imaging. 2010;21(2):101-113. doi:10.1097/RMR.0b013e31821e56d8.

63. Adler S, Lorio S, Jacques TS, et al. Towards in vivo focal cortical dysplasia phenotyping using quantitative MRI. Neuroimage Clin. 2017;15:95-105. doi:10.1016/j.nicl.2017.04.017.

64. Filo S, Mezer Aviv A. PD: Proton Density of Tissue Water. In: Tofts P, Dowell NG, Cercignani M, eds. Quantitative MRI of the brain. Principles of physical measurement. Boca Raton, London, New York: CRC Press Taylor \& Francis Group; 2018:55-71.

65. Shah NJ, Neeb H, Kircheis G, Engels P, Häussinger D, Zilles K. Quantitative cerebral water content mapping in hepatic encephalopathy. Neuroimage. 2008;41(3):706-717. doi:10.1016/j.neuroimage.2008.02.057.

66. Brex PA, Parker GJ, Leary SM, et al. Lesion heterogeneity in multiple sclerosis. A study of the relations between appearances on T1 weighted images, T1 relaxation times, and metabolite concentrations. J Neurol Neurosurg Psychiatry. 2000;68(5):627-632. 
67. Rooney WD, Johnson G, Li X, et al. Magnetic field and tissue dependencies of human brain longitudinal $1 \mathrm{H} 2 \mathrm{O}$ relaxation in vivo. Magn Reson Med. 2007;57(2):308-318. doi:10.1002/mrm.21122.

68. Stüber C, Morawski M, Schäfer A, et al. Myelin and iron concentration in the human brain. A quantitative study of MRI contrast. Neuroimage. 2014;93 Pt 1:95-106. doi:10.1016/j.neuroimage.2014.02.026.

69. Gracien R-M, Reitz SC, Hof SM, et al. Changes and variability of proton density and $\mathrm{T} 1$ relaxation times in early multiple sclerosis. MRI markers of neuronal damage in the cerebral cortex. Eur Radiol. 2016;26(8):2578-2586. doi:10.1007/s00330-015-4072-x.

70. Laule C, Vavasour IM, Moore GRW, et al. Water content and myelin water fraction in multiple sclerosis. A T2 relaxation study. J Neurol. 2004;251(3):284-293. doi:10.1007/s00415-004-0306-6.

71. Whittall KP, MacKay AL, Graeb DA, Nugent RA, Li DK, Paty DW. In vivo measurement of T2 distributions and water contents in normal human brain. Magn Reson Med. 1997;37(1):34-43.

72. Blystad I, Warntjes JBM, Smedby Ö, Lundberg P, Larsson E-M, Tisell A. Quantitative MRI for analysis of peritumoral edema in malignant gliomas. PLOS ONE. 2017;12(5):e0177135. doi:10.1371/journal.pone.0177135.

73. Chavhan GB, Babyn PS, Thomas B, Shroff MM, Haacke EM. Principles, techniques, and applications of T2*-based MR imaging and its special applications. Radiographics. 2009;29(5):1433-1449. doi:10.1148/rg.295095034.

74. Vymazal J, Hajek M, Patronas N, et al. The quantitative relation between T1-weighted and T2-weighted MRI of normal gray matter and iron concentration. J Magn Reson Imaging. 1995;5(5):554-560. 
75. Haacke EM, Cheng NYC, House MJ, et al. Imaging iron stores in the brain using magnetic resonance imaging. Magn Reson Imaging. 2005;23(1):1-25. doi:10.1016/j.mri.2004.10.001.

76. Ashburner J, Friston KJ. Voxel-based morphometry--the methods. Neuroimage. 2000;11(6 Pt 1):805-821. doi:10.1006/nimg.2000.0582.

77. Zoons E, Booij J, Nederveen AJ, Dijk JM, Tijssen MAJ. Structural, functional and molecular imaging of the brain in primary focal dystonia--a review. Neuroimage. 2011;56(3):1011-1020. doi:10.1016/j.neuroimage.2011.02.045.

78. Etgen T, Mühlau M, Gaser C, Sander D. Bilateral grey-matter increase in the putamen in primary blepharospasm. $J$ Neurol Neurosurg Psychiatry. 2006;77(9):1017-1020. doi:10.1136/jnnp.2005.087148.

79. Black KJ, Ongür D, Perlmutter JS. Putamen volume in idiopathic focal dystonia. Neurology. 1998;51(3):819-824.

80. Obermann M, Yaldizli O, Greiff A de, et al. Morphometric changes of sensorimotor structures in focal dystonia. Mov Disord. 2007;22(8):1117-1123. doi:10.1002/mds.21495.

81. Pantano $P$, Totaro $P$, Fabbrini $G$, et al. A transverse and longitudinal MR imaging voxel-based morphometry study in patients with primary cervical dystonia. AJNR Am J Neuroradiol. 2011;32(1):81-84. doi:10.3174/ajnr.A2242.

82. Zheng Z, Pan P, Wang W, Shang $\mathrm{H}$. Neural network of primary focal dystonia by an anatomic likelihood estimation meta-analysis of gray matter abnormalities. J Neurol Sci. 2012;316(1-2):51-55. doi:10.1016/j.jns.2012.01.032.

83. Draganski B, Thun-Hohenstein C, Bogdahn U, Winkler J, May A. "Motor circuit" gray matter changes in idiopathic cervical dystonia. Neurology. 2003;61(9):1228-1231. 
84. Egger K, Mueller J, Schocke M, et al. Voxel based morphometry reveals specific gray matter changes in primary dystonia. Mov Disord. 2007;22(11):1538-1542. doi:10.1002/mds.21619.

85. Delmaire C, Vidailhet M, Elbaz A, et al. Structural abnormalities in the cerebellum and sensorimotor circuit in writer's cramp. Neurology. 2007;69(4):376-380. doi:10.1212/01.wnl.0000266591.49624.1a.

86. Garraux G, Bauer A, Hanakawa T, Wu T, Kansaku K, Hallett M. Changes in brain anatomy in focal hand dystonia. Ann Neurol. 2004;55(5):736-739. doi:10.1002/ana.20113.

87. Fabbrini G, Pantano P, Totaro P, et al. Diffusion tensor imaging in patients with primary cervical dystonia and in patients with blepharospasm. Eur J Neurol. 2008;15(2):185-189. doi:10.1111/j.1468-1331.2007.02034.x.

88. Carbon M, Kingsley PB, Tang C, Bressman S, Eidelberg D. Microstructural white matter changes in primary torsion dystonia. Mov Disord. 2008;23(2):234-239. doi:10.1002/mds.21806.

89. Beaulieu C. The basis of anisotropic water diffusion in the nervous system a technical review. NMR Biomed. 2002;15(7-8):435-455. doi:10.1002/nbm.782.

90. Colosimo C, Pantano P, Calistri V, Totaro P, Fabbrini G, Berardelli A. Diffusion tensor imaging in primary cervical dystonia. J Neurol Neurosurg Psychiatry. 2005;76(11):1591-1593. doi:10.1136/jnnp.2004.056614.

91. Aschermann Z, Perlaki G, Orsi G, et al. Quantitative assessment of brain iron by $\mathrm{R}^{*}$ relaxometry in patients with cervical dystonia. Mov Disord. 2015;30(10):1422-1426. doi:10.1002/mds.26306.

92. Schneider S, Feifel E, Ott D, Schumacher M, Lücking CH, Deuschl G. Prolonged MRI T2 times of the lentiform nucleus in idiopathic spasmodic torticollis. Neurology. 1994;44(5):846-850. 
93. Jost WH, Hefter H, Stenner A, Reichel G. Rating scales for cervical dystonia. A critical evaluation of tools for outcome assessment of botulinum toxin therapy. J Neural Transm (Vienna). 2013;120(3):487-496. doi:10.1007/s00702-012-0887-7.

94. Tsui JK, Eisen A, Stoessl AJ, Calne S, Calne DB. Double-blind study of botulinum toxin in spasmodic torticollis. Lancet. 1986;2(8501):245-247.

95. Consky ES, Lang AE. Clinical assessments of patients with cervical dystonia. In: Jankovic J, Hallett M, eds. Therapy with botulinum toxin. New York: M. Dekker; 1994:211-237.

96. Jankovic J, Orman J. Botulinum A toxin for cranial-cervical dystonia. A double-blind, placebo-controlled study. Neurology. 1987;37(4):616-623.

97. Jankovic J, Kenney C, Grafe S, Goertelmeyer R, Comes G. Relationship between various clinical outcome assessments in patients with blepharospasm. Mov Disord. 2009;24(3):407-413. doi:10.1002/mds.22368.

98. Wissel J, Kabus C, Wenzel R, et al. Botulinum toxin in writer's cramp. Objective response evaluation in 31 patients. J Neurol Neurosurg Psychiatry. 1996;61(2):172-175.

99. Merz RI, Deakin J, Hawthorne MR. Oromandibular dystonia questionnaire (OMDQ-25). A valid and reliable instrument for measuring health-related quality of life. Clin Otolaryngol. 2010;35(5):390-396. doi:10.1111/j.17494486.2010.02194.x.

100. Preibisch C, Deichmann R. T1 mapping using spoiled FLASH-EPI hybrid sequences and varying flip angles. Magn Reson Med. 2009;62(1):240-246. doi:10.1002/mrm.21969.

101. Magerkurth J, Volz S, Wagner M, et al. Quantitative $T^{\star} 2$-mapping based on multi-slice multiple gradient echo flash imaging: retrospective correction 
for subject motion effects. Magn Reson Med. 2011;66(4):989-997. doi:10.1002/mrm.22878.

102. Nöth U, Volz S, Hattingen E, Deichmann R. An improved method for retrospective motion correction in quantitative $\mathrm{T}^{*}$ mapping. Neuroimage. 2014;92:106-119. doi:10.1016/j.neuroimage.2014.01.050.

103. Preibisch C, Deichmann R. Influence of RF spoiling on the stability and accuracy of T1 mapping based on spoiled FLASH with varying flip angles. Magn Reson Med. 2009;61(1):125-135. doi:10.1002/mrm.21776.

104. Volz S, Nöth U, Deichmann R. Correction of systematic errors in quantitative proton density mapping. Magn Reson Med. 2012;68(1):74-85. doi:10.1002/mrm.23206.

105. Volz S, Nöth U, Jurcoane A, Ziemann U, Hattingen E, Deichmann R. Quantitative proton density mapping. Correcting the receiver sensitivity bias via pseudo proton densities. Neuroimage. 2012;63(1):540-552. doi:10.1016/j.neuroimage.2012.06.076.

106. Nöth U, Hattingen E, Bähr O, Tichy J, Deichmann R. Improved visibility of brain tumors in synthetic MP-RAGE anatomies with pure T1 weighting. NMR Biomed. 2015;28(7):818-830. doi:10.1002/nbm.3324.

107. Fischl B, Salat DH, Busa E, et al. Whole brain segmentation: automated labeling of neuroanatomical structures in the human brain. Neuron. 2002;33(3):341-355.

108. Smith SM, Jenkinson M, Woolrich MW, et al. Advances in functional and structural MR image analysis and implementation as FSL. Neuroimage. 2004;23 Suppl 1:S208-19. doi:10.1016/j.neuroimage.2004.07.051.

109. Winkler AM, Ridgway GR, Webster MA, Smith SM, Nichols TE. Permutation inference for the general linear model. Neuroimage. 2014;92:381-397. doi:10.1016/j.neuroimage.2014.01.060. 
110. Prudente CN, Stilla R, Buetefisch CM, et al. Neural Substrates for Head Movements in Humans: A Functional Magnetic Resonance Imaging Study. $J$ Neurosci. 2015;35(24):9163-9172. doi:10.1523/JNEUROSCI.0851-15.2015.

111. Langkammer C, Krebs N, Goessler W, et al. Quantitative MR imaging of brain iron: a postmortem validation study. Radiology. 2010;257(2):455-462. doi:10.1148/radiol.10100495.

112. Novellino F, Cherubini A, Chiriaco $C$, et al. Brain iron deposition in essential tremor. A quantitative 3-Tesla magnetic resonance imaging study. Mov Disord. 2013;28(2):196-200. doi:10.1002/mds.25263.

113. Banerjee A, Chitnis UB, Jadhav SL, Bhawalkar JS, Chaudhury S. Hypothesis testing, type I and type II errors. Ind Psychiatry J. 2009;18(2):127131. doi:10.4103/0972-6748.62274.

114. Ethofer T, Mader I, Seeger U, et al. Comparison of longitudinal metabolite relaxation times in different regions of the human brain at 1.5 and 3 Tesla. Magn Reson Med. 2003;50(6):1296-1301. doi:10.1002/mrm.10640.

115. Abbas Z, Gras V, Möllenhoff K, Keil F, Oros-Peusquens A-M, Shah NJ. Analysis of proton-density bias corrections based on $\mathrm{T} 1$ measurement for robust quantification of water content in the brain at 3 Tesla. Magn Reson Med. 2014;72(6):1735-1745. doi:10.1002/mrm.25086.

116. Gelman N, Ewing JR, Gorell JM, Spickler EM, Solomon EG. Interregional variation of longitudinal relaxation rates in human brain at 3.0 T: relation to estimated iron and water contents. Magn Reson Med. 2001;45(1):71-79.

117. Gracien R-M, Jurcoane A, Wagner M, et al. Multimodal quantitative MRI assessment of cortical damage in relapsing-remitting multiple sclerosis. $J$ Magn Reson Imaging. 2016;44(6):1600-1607. doi:10.1002/jmri.25297.

118. Gracien R-M, Jurcoane A, Wagner M, et al. The Relationship between Gray Matter Quantitative MRI and Disability in Secondary Progressive 
Multiple Sclerosis. PLoS ONE. 2016;11(8):e0161036. doi:10.1371/journal.pone. 0161036 .

119. Brück W, Bitsch A, Kolenda H, Brück Y, Stiefel M, Lassmann H. Inflammatory central nervous system demyelination: correlation of magnetic resonance imaging findings with lesion pathology. Ann Neurol. 1997;42(5):783793. doi:10.1002/ana.410420515.

120. Wick W, Küker W. Brain edema in neurooncology: radiological assessment and management. Onkologie. 2004;27(3):261-266. doi:10.1159/000077976.

121. Ayata C, Ropper AH. Ischaemic brain oedema. J Clin Neurosci. 2002;9(2):113-124. doi:10.1054/jocn.2001.1031.

122. García-Albea E, Franch O, Muñoz D, Ricoy JR. Brueghel's syndrome, report of a case with postmortem studies. J Neurol Neurosurg Psychiatry. 1981;44(5):437-440. doi:10.1136/jnnp.44.5.437.

123. LeDoux MS, Brady KA. Secondary cervical dystonia associated with structural lesions of the central nervous system. Mov Disord. 2003;18(1):60-69. doi:10.1002/mds.10301.

124. Jinnah HA, Neychev V, Hess EJ. The Anatomical Basis for Dystonia: The Motor Network Model. Tremor Other Hyperkinet Mov (N Y). 2017;7:506. doi:10.7916/D8V69X3S.

125. Bonilha L, Vries PM de, Vincent DJ, et al. Structural white matter abnormalities in patients with idiopathic dystonia. Mov Disord. 2007;22(8):11101116. doi:10.1002/mds.21295.

126. Berman BD, Honce JM, Shelton E, Sillau SH, Nagae LM. Isolated focal dystonia phenotypes are associated with distinct patterns of altered microstructure. Neuroimage Clin. 2018;19:805-812. doi:10.1016/j.nicl.2018.06.004. 
127. Piccinin CC, Piovesana LG, Santos MCA, et al. Diffuse decreased gray matter in patients with idiopathic craniocervical dystonia: a voxel-based morphometry study. Front Neurol. 2014;5:283. doi:10.3389/fneur.2014.00283.

128. Tarsy D. Comparison of clinical rating scales in treatment of cervical dystonia with botulinum toxin. Mov Disord. 1997;12(1):100-102. doi:10.1002/mds.870120117.

129. Albanese A, Sorbo FD, Comella $\mathrm{C}$, et al. Dystonia rating scales: critique and recommendations. Mov Disord. 2013;28(7):874-883. doi:10.1002/mds.25579.

130. Landman BA, Huang AJ, Gifford A, et al. Multi-parametric neuroimaging reproducibility: a 3-T resource study. Neuroimage. 2011;54(4):2854-2866. doi:10.1016/j.neuroimage.2010.11.047.

131. Wyss M, Duerst $Y$, Nanz D, et al. Feedback field control improves the precision of T2 * quantification at 7 T. NMR Biomed. 2017;30(10). doi:10.1002/nbm.3753.

132. Battistella G, Termsarasab P, Ramdhani RA, Fuertinger S, Simonyan K. Isolated Focal Dystonia as a Disorder of Large-Scale Functional Networks. Cereb Cortex. 2017;27(2):1203-1215. doi:10.1093/cercor/bhv313.

133. Lehéricy S, Tijssen MAJ, Vidailhet M, Kaji R, Meunier S. The anatomical basis of dystonia: current view using neuroimaging. Mov Disord. 2013;28(7):944-957. doi:10.1002/mds.25527.

134. Dresel C, Li Y, Wilzeck V, Castrop F, Zimmer C, Haslinger B. Multiple changes of functional connectivity between sensorimotor areas in focal hand dystonia. J Neurol Neurosurg Psychiatry. 2014;85(11):1245-1252. doi:10.1136/jnnp-2013-307127. 
135. Jochim A, Li Y, Gora-Stahlberg G, et al. Altered functional connectivity in blepharospasm/orofacial dystonia. Brain Behav. 2018;8(1):e00894. doi:10.1002/brb3.894.

136. Nevrlý $M$, Hluštík $P$, Hok $P$, Otruba $P$, Tüdös $Z$, Kaňovský $P$. Changes in sensorimotor network activation after botulinum toxin type $A$ injections in patients with cervical dystonia: a functional MRI study. Exp Brain Res. 2018;236(10):2627-2637. doi:10.1007/s00221-018-5322-3.

137. Simonyan K, Frucht SJ, Blitzer A, Sichani AH, Rumbach AF. A novel therapeutic agent, sodium oxybate, improves dystonic symptoms via reduced network-wide activity. Sci Rep. 2018;8(1):16111. doi:10.1038/s41598-018-34553-x.

138. Pettigrew LC, Jankovic J. Hemidystonia: a report of 22 patients and a review of the literature. J Neurol Neurosurg Psychiatry. 1985;48(7):650-657.

139. Scott BL, Jankovic J. Delayed-onset progressive movement disorders after static brain lesions. Neurology. 1996;46(1):68-74. 


\section{Anhang}

\section{A.1 Toronto Western Spasmodic Torticollis Rating Scale (TWSTRS) ${ }^{95}$}

\begin{tabular}{lllllll}
\hline I Schweregrad des Torticollis (maximal 35 Punkte) & & & & & \\
\hline A. Maximale Beweglichkeit & 0 & 1 & 2 & 3 & 4 & \\
1. Rotation & 0 & 1 & 2 & 3 & & \\
2. Laterocollis & 0 & 1 & 2 & 3 & & \\
3. Antero-/Retrocollis & 0 & 1 & & & & \\
4. Laterale Verschiebung & 0 & 1 & & & & \\
$\quad$ 5. Sagittale Verschiebung & 0 & 1 & 2 & 3 & 4 & 5 \\
B. Dauer (doppelt gewichtet) & 0 & 1 & 2 & & & \\
C. Effekt von sensorischen Tricks & 0 & 1 & 2 & 3 & & \\
D. Schulterelevation/-anteversion & 0 & 1 & 2 & 3 & 4 & \\
E. Ausmaß der Beweglichkeit & 0 & 1 & 2 & 3 & 4 &
\end{tabular}

\section{Grad der Behinderung (maximal 30 Punkte)}

$\begin{array}{lllllll}\text { A. Arbeit } & 0 & 1 & 2 & 3 & 4 & 5 \\ \text { B. Aktivitäten des täglichen Lebens } & 0 & 1 & 2 & 3 & 4 & 5 \\ \text { C. Autofahren } & 0 & 1 & 2 & 3 & 4 & 5 \\ \text { D. Lesen } & 0 & 1 & 2 & 3 & 4 & 5 \\ \text { E. Fernsehen } & 0 & 1 & 2 & 3 & 4 & 5 \\ \text { F. Aktivitäten außerhalb des Hauses } & 0 & 1 & 2 & 3 & 4 & 5\end{array}$

III Schmerzskala (maximal 20 Punkte)
A. Schweregrad des Schmerzes (VAS-Skala)
(schlimmster Schmerz + günstigster Schmerz + (2*üblicher Schmerz) $) / 4$

B. Dauer des Schmerzes

C. Behinderung durch den Schmerz

$\begin{array}{llllll}0 & 1 & 2 & 3 & 4 & 5\end{array}$




\section{A.2 Writer's Cramp Rating Scale ${ }^{98}$}

\begin{tabular}{|c|c|c|c|}
\hline \multicolumn{4}{|c|}{ Part A: writing movement score } \\
\hline \multicolumn{4}{|c|}{ 1. Dystone Haltung ${ }^{\star}$} \\
\hline \multicolumn{2}{|l|}{ pathologische Flexion } & \multicolumn{2}{|c|}{ pathologische Extension } \\
\hline 0 & keine & 0 & \\
\hline 1 & moderat & 1 & \\
\hline 2 & deutlich & 2 & ES (0-2) \\
\hline \multicolumn{4}{|l|}{ Hangelenksscore (WRS) } \\
\hline \multicolumn{2}{|l|}{ pathologische Flexion } & \multicolumn{2}{|c|}{ pathologische Extension } \\
\hline 0 & keine & 0 & \\
\hline 1 & moderat & 1 & \\
\hline 2 & deutlich & 2 & \\
\hline \multicolumn{2}{|c|}{ pathologische Ulnarabduktion } & \multicolumn{2}{|c|}{ pathologische Radialabduktion } \\
\hline 0 & keine & 0 & \\
\hline 1 & moderat & 1 & \\
\hline 2 & deutlich & 2 & WRS $(0-4)$ \\
\hline \multicolumn{4}{|l|}{ Fingerscore (FS) } \\
\hline \multicolumn{4}{|l|}{ Daumen } \\
\hline pathologische Flexion & & \multicolumn{2}{|c|}{ pathologische Extension } \\
\hline 0 & keine & 0 & \\
\hline 1 & moderat & 1 & \\
\hline 2 & deutlich & 2 & \\
\hline \multicolumn{4}{|l|}{ Zeigefinger } \\
\hline \multicolumn{2}{|l|}{ pathologische Flexion } & \\
\hline 0 & keine & \multicolumn{2}{|c|}{ pathologische Extension } \\
\hline 1 & moderat & \multicolumn{2}{|l|}{1} \\
\hline 2 & deutlich & \multicolumn{2}{|l|}{2} \\
\hline \multicolumn{4}{|l|}{ Mittelfinger } \\
\hline pathologische Flexion & & \multicolumn{2}{|c|}{ pathologische Extension } \\
\hline 0 & keine & 0 & \\
\hline 1 & moderat & 1 & \\
\hline 2 & deutlich & 2 & FS $(0-6)$ \\
\hline \multicolumn{4}{|c|}{ 2. Latenz der Dystonie (L) } \\
\hline \multirow{2}{*}{\multicolumn{2}{|c|}{$\begin{array}{l}\text { mindestens drei Buchstaben möglich } \\
\text { mit Beginn des Schreibens }\end{array}$}} & 1 & \\
\hline & & 2 & $L(1-2)$ \\
\hline \multicolumn{4}{|c|}{ 3. Schreibtremor (WT) } \\
\hline & \multicolumn{2}{|l|}{0} \\
\hline moderater Schreibtremc & & 1 & \\
\hline schwerer Schreibtremor & & 2 & WT $(0-2)$ \\
\hline Part B: writing speed & & & \\
\hline Schriftgeschwindigkei & & & \\
\hline norm & & 0 & \\
\hline leichtgradig ve & samt & 1 & \\
\hline hochgradig ve & samt & 2 & WS $(0-2)$ \\
\hline
\end{tabular}


* Nach einer Studie mit 24 gesunden Probanden wird folgende „normale Schreibhaltung“ definiert: Ellenbogenflexion circa $90^{\circ}$, Neutralposition des Handgelenkes und Flexion von circa $25^{\circ}$ in den Fingergelenken des Daumens, Zeige- und Mittelfingers.

Die "Writer's Cramp Writing Scale" besteht aus zwei Subscores, dem writing movement score (Part A) und dem writing speed subscore (Part B).

Writing movement score $=(E S+W R S+F S) \times L+W T \times 2 \quad(0-28$ Punkte $)$

Writing speed score $=$ WS

(0 - 2 Punkte) 


\section{Eigene Veröffentlichungen}

Gracien RM, Petrov F, Hok P, van Wijnen A, Maiworm M, Seiler A, Deichmann R, Baudrexel S. Multimodal Quantitative MRI Reveals No Evidence for Tissue Pathology in Idiopathic Cervical Dystonia. Front Neurol. 2019 Aug 27;10:914. doi: 10.3389/fneur.2019.00914.

Gracien RM, van Wijnen A, Maiworm M, Petrov F, Merkel N, Paule E, Steinmetz H, Knake S, Rosenow F, Wagner M, Deichmann R. Improved synthetic T1weighted images for cerebral tissue segmentation in neurological diseases. Magn Reson Imaging. 2019 Sep;61:158-166. doi: 10.1016/j.mri.2019.05.013.

van Wijnen A, Petrov F, Maiworm M, Frisch S, Foerch C, Hattingen E, Steinmetz H, Klein JC, Deichmann R, Wagner M, Gracien RM. Cortical quantitative MRI parameters are related to the cognitive status in patients with relapsing-remitting multiple sclerosis. Eur Radiol. 2020 Feb;30(2):1045-1053. doi: 10.1007/s00330019-06437-9. 


\section{Lebenslauf}

[Von der Veröffentlichung des Lebenslaufes wurde entsprechend der Vorgaben des Promotionsbüro abgesehen.] 


\section{Danksagung}

An dieser Stelle möchte ich allen beteiligten Personen danken, die mich bei der Anfertigung meiner Dissertation unterstützt haben.

Mein besonderer Dank gilt PD Dr. Simon Baudrexel für die hervorragende Betreuung meiner Arbeit und die Bereitstellung des Themas. Auch für die mühevolle Arbeit des Korrekturlesens möchte ich mich bei ihm herzlich bedanken.

Zudem danke ich PD Dr. René-Maxime Gracien für die große Unterstützung bei der Durchführung sowie Umsetzung der gesamten Arbeit. Danke für die Einweisung in die Thematik sowie die große Hilfe bei der Auswertung der MRT-Daten.

Besonderen Dank auch an die gesamte Arbeitsgruppe im BIC, insbesondere an Prof. Ralf Deichmann, für die stete Hilfsbereitschaft und freundliche Arbeitsatmosphäre, die zum Gelingen dieser Arbeit beigetragen haben.

Danke an Alexandra van Wijnen und Michelle Maiworm für die vielen gemeinsamen Stunden im Scanner.

Auch danke ich den Mitarbeitern der Bewegungsambulanz der Uniklinik Frankfurt für die herzliche Aufnahme ins Team und der Mithilfe bei der Rekrutierung.

Mein Dank gilt nicht zuletzt meinen Eltern, meinem Ehemann, meiner Familie und meinen Freunden, die mich während meiner gesamten Ausbildung unterstützt und mir zu jeder Zeit mit Rat und Tat zur Seite standen. 


\section{Schriftliche Erklärung}

Ich erkläre ehrenwörtlich, dass ich die dem Fachbereich Medizin der Johann Wolfgang Goethe-Universität Frankfurt am Main zur Promotionsprüfung eingereichte Dissertation mit dem Titel

\section{Charakterisierung mikrostruktureller zerebraler Pathologie bei fokaler idiopathischer Dystonie mittels quantitativer Magnetresonanztomographie}

im Zentrum der Neurologie und Neurochirurgie (ZNN), Klinik für Neurologie, des Universitätsklinikums Frankfurt unter Betreuung und Anleitung von PD Dr. Simon Baudrexel mit Unterstützung durch PD Dr. René-Maxime Gracien ohne sonstige Hilfe selbst durchgeführt und bei der Abfassung der Arbeit keine anderen als die in der Dissertation angeführten Hilfsmittel benutzt habe. Darüber hinaus versichere ich, nicht die Hilfe einer kommerziellen Promotionsvermittlung in Anspruch genommen zu haben.

Ich habe bisher an keiner in- oder ausländischen Universität ein Gesuch um Zulassung zur Promotion eingereicht. Die vorliegende Arbeit wurde bisher nicht als Dissertation eingereicht.

Vorliegende Ergebnisse der Arbeit wurden in folgendem Publikationsorgan veröffentlicht:

Gracien RM, Petrov F, Hok P, van Wijnen A, Maiworm M, Seiler A, Deichmann R, Baudrexel S. Multimodal Quantitative MRI Reveals No Evidence for Tissue Pathology in Idiopathic Cervical Dystonia. Frontiers in Neurology, Ausgabe 10, Seite 914, 2019. 